\title{
Numerical Modeling of Fluid-Structure Interaction in Arteries with Anisotropic Polyconvex Hyperelastic and Anisotropic Viscoelastic Material Models at Finite Strains
}

\author{
D. Balzani ${ }^{1}$, S. Deparis ${ }^{2}$, S. Fausten ${ }^{3}$, D. Forti ${ }^{2}$, A. Heinlein ${ }^{4}$, A. Klawonn ${ }^{4}$, A. Quarteroni ${ }^{2,5}$, \\ O. Rheinbach ${ }^{6}$, and J. Schröder ${ }^{3}$
}

\begin{abstract}
The distribution of stresses in arterial walls under physiological loading conditions is considered; transmural stress distributions are a major driving factor for atherogenesis. The accurate prediction of transmural stresses requires the use of a model for the vessel wall that is able to capture the relevant features of the material behavior. An anisotropic hyperelastic and almost incompressible material model is considered, based on a polyconvex strain energy function. One of the main contributions of this paper is the use of a highly nonlinear, polyconvex anisotropic structural model for the solid in the context of fluid-structure interaction (FSI), together with a suitable discretization. Additionally, the influence of viscoelasticity is investigated. Realistic predictions of transmural stress distributions require the simulation of the interaction between the blood flow and the vessel wall deformation. The fluid-structure interaction problem is solved using a monolithic approach, i.e., the nonlinear system is solved (after time and space discretization) as a whole without splitting among its components. The linearized block systems are solved iteratively using parallel domain decomposition preconditioners. A simple - but nonsymmetric - curved geometry is proposed which is suitable as a benchmark testbed for fluid-structure interaction simulations in biomechanics where nonlinear structural models are used. To incorporate the prestretch of the arterial wall due to the blood pressure, a ramp phase is used to bring the setting to a physiological blood pressure of $80 \mathrm{mmHg}$, followed by a simulation of one or several heartbeats. We propose this benchmark to replace, for problems in biomechanics, the simpler problem of a short and steep wave in a straight tube, which is often used as a first test problem in FSI. Based on the curved benchmark geometry, and corresponding physiologicallybased boundary conditions, the influence of different material models, spatial discretizations, and meshes of varying refinement is investigated. As a result of the almost incompressibility, linear shape functions in the structure are not sufficient to provide good approximations of the arterial wall stresses. This holds although reasonably fine discretizations using linear finite elements seem to provide good approximations of the wall displacements. Instead, suitable results are obtained by considering at least piecewise quadratic shape functions for the deformations. In addition, based on a piecewise quadratic discretization of the displacements, a discretization using a three-field approach and element-by-element static condensation of two of the three fields is applied in order to avoid locking effects. The results obtained are similar

\footnotetext{
1 Institute of Mechanics and Shell Structures, Technical University Dresden, 01062 Dresden, Germany

2 Chair of Modeling and Scientific Computing, MATHICSE - EPFL, Avenue Piccard, Station 8, 1015 Lausanne, Switzerland

3 Institute of Mechanics, University of Duisburg-Essen, Department of Engineering, Universitätsstraße 15, 45141 Essen, Germany

4 Mathematisches Institut, Universität zu Köln, Weyertal 86-90, 50931 Köln, Germany

5 MOX, Politecnico di Milano, via Bonardi 9, 20133 Milan, Italy (on leave)

${ }^{6}$ Institute of Numerical Mathematics and Optimization, Technische Universität Bergakademie Freiberg, Akademiestraße 6, 09599 Freiberg, Germany
} 
to those from using standard piecewise quadratic finite elements. For the time discretization, a secondorder BDF scheme is used. It turns out that the curved geometry enables the analysis of non-rotationally symmetric distributions of the mechanical fields. For instance, the maximal shear stresses in the fluidstructure interface are found to be higher in the inner curve which corresponds to clinical observations indicating a high plaque nucleation probability at such locations.

\section{Introduction}

Computational simulation of diseased arteries represents a novel approach in clinical diagnosis and treatment assistance provided that accurate predictions of the mechanical behavior are available. They may not only help to optimize medical methods of treatment, they may also enable a more precise assessment whether the decision for no surgical intervention is justified or not. In particular transmural wall stresses are expected to provide important information for an estimation whether, e.g., an atherosclerotic plaque is likely to rupture if the artery is not treated. Therefore, reliable material models as well as robust numerical methods are necessary to provide simulations that allow for a realistic prediction of these stresses. Arterial tissue is composed of an elastin-rich ground substance with embedded collagen and smooth muscle cells. This composition yields an anisotropic and viscoelastic material response at finite strains. We thus consider the fluid-structure interaction (FSI) using sophisticated material models for the structure.

To model the hyperelastic response of the structure various models have been proposed in the literature, however the essential condition of polyconvexity [3] was considered only since approximately 10 years. This is due to the fact that the first anisotropic polyconvex functions were just introduced in [71]. Based thereon, [10] constructed a variety of polyconvex functions which a priori satisfied the condition of a stressfree reference configuration. They were compared in [20] with respect to their numerical performance and their performance using parallel iterative solvers, i.e., the FETI-DP domain decomposition method. A larger structural simulation of an arterial wall for a diseased artery using one of these anisotropic, almost incompressible hyperelastic material models was then been presented in [53], applying a Newton-Krylov FETI-DP approach. Anisotropy, to model embedded collagen fibers, is one of the numerical challenges present in models for soft biological tissue. It was observed numerically in [67] and [7] that the anisotropy of soft tissue does clearly affect Newton's method as well as the iterative linear solver but that the effect is, in the physiological range, not severe. Damage of the fibers from overstretch $[4,11]$ has been considered in computations with the FETI-DP method, for an arterial segment, in [66, 6]. It was observed that it poses no additional challenge to the solver of the linearized system as it rather decreases the effect of the anisotropy.

Using these results, patient-specific simulations of arteries, neglecting the influence of the blood-flow and a viscoelastic material behavior, were shown in [5]. Viscoelastic effects in fluid-structure interaction, using reduced models, were already considered and compared with experiments in [23]. In [72], it was found that the well-known model of [48] also fulfills the polyconvexity condition. A model that goes beyond the concept of hyperelasticity and which includes also the viscoelastic material behavior of arteries is given in [47]. This approach is mainly based on the original framework for viscoelasticity at finite strains in [73]. However, the formulation is restricted to a volumetric-isochoric split of the strain energy function and therefore it allows for stresses induced in the fibers by a volumetric strain; cf. [70]. Furthermore, the viscoelastic behavior is not only associated with the smooth muscle cells as it considers overstresses in the complete isochoric part including the response of the elastin matrix. A unified approach for the inelastic response of arterial tissues is given in [51].

Aside from the mechanical behavior of the arterial tissue itself, stresses therein depend strongly on the interaction with the blood flow imposing a complex and inhomogeneous shear stress and pressure distribution on the inner vessel wall surface. Therefore, the fluid-structure interaction is important to be considered in numerical computations. Recently, in [79], FSI in idealized healthy cerebral arteries with both, 
nonlinear isotropic and anisotropic material constitutive laws was investigated, with particular highlight on the role of the fibers. In [79], P1 finite elements were used to represent the structure displacements for a non-polyconvex hyperelastic energy, but a grid convergence analysis was not reported.

In the present article, we rather follow a systematic approach with a special focus on the structural side of the simulation. First, with respect to the modeling of the structure, we consider polyconvex hyperelastic energies. This current paper continues the earlier work started in [8], where, to the best of our knowledge, for the first time a polyconvex anisotropic hyperelastic energy was used in FSI. Furthermore, in this paper, we also consider viscoelasticity in FSI. Second, with respect to the space discretization of the structure, beyond simple P1 finite elements, we consider P2 as well as $\overline{\mathrm{F}}$ finite elements. The latter are based on a three-field formulation [74], to avoid locking from the incompressibility constraint. This is important to obtain a good estimate of the stresses in the arterial wall. Third, we perform mesh convergence studies using all different space discretizations. Our smallest mesh has only 30000 total degrees of freedom whereas the largest one has over a million total degrees of freedom; cf. Table 4 in Section 4.1.4. To incorporate the necessary prestretch from the blood pressure, we use a ramp phase before applying one or several heart beats. We discuss the shape of the ramp and possible sources of (decaying) oscillations observed, e.g., in the pressure. We also take special care to use a suitable absorbing boundary condition at the outflow; see Section 4.1.5 and Section 4.2.5.

We consider a fully-coupled monolithic algorithm to solve the fluid-structure interaction problem in an idealized artery. We model the fluid dynamics by the Navier-Stokes equations in Arbitrary-Lagrangian Eulerian (ALE) coordinates $[15,26,43]$ and the structure by nonlinear anisotropic material laws in a Lagrangian frame of reference. The FSI problem is composed of three subproblems, i.e., the fluid, the structure, and the geometry problems. At the fluid-structure interface we enforce the geometry adherence between the fluid and structure displacement fields, the continuity of the velocities, and the equilibrium of the normal stresses. The system of equations describing the FSI problem is treated as a single system involving all the state variables in a monolithic fashion. In general, this system is nonlinear because of the convective term in the Navier-Stokes equations, the nonlinearity of the constitutive law of the structure, and the moving fluid domain.

The FSI system is discretized by the finite element method in space and by finite differences in time, using $\overline{\mathrm{F}}$, i.e., $P_{2}-P_{0}-P_{0}$, finite elements for the structure, $P_{2}-P_{1}$ finite elements for the fluid, see Section 3.2, and a second order BDF-scheme in time, see Section 4.1.3. Conforming discretizations at the fluid-structure interface are considered.

A monolithic Convective Explicit (CE) algorithm, for which the convective term of the fluid momentum is linearized by temporal extrapolation, is used for solving the fully coupled FSI problem $[2,26]$. The resulting discretized nonlinear FSI problem is solved by Newton's method wherein at each nonlinear iteration, the linearized FSI system is solved in parallel by the GMRES method preconditioned by an approximated monolithic Dirichlet-Neumann preconditioner [26].

\subsection{Fluid-Structure Interaction Algorithms in Biomechanics}

Extensive work has been carried out on the development of algorithms for the solution of time-dependent FSI problems in biomechanics. The approaches are typically categorized either as segregated or as monolithic coupling schemes. However, the distinction is not always straight forward. Segregated schemes can range from loosely coupled iterative schemes, such as simple, possibly accelerated, fixed point iterations, to schemes with a much stronger coupling which still use separate solvers for fluid and structure. Monolithic schemes range from block preconditioners for the fully coupled problem constructed from segregated solvers to preconditioners which are not constructed from separate solvers.

Loosely coupled FSI algorithms were studied, e.g., by Causin, Gerbeau, and Nobile in [24]. In Deparis, Discacciati, Fourestey, and Quarteroni [30] methods based on Schur complement approaches were 
studied. Whereas in the algorithms in [30] the nonlinear Steklov-Poincaré operators are linearized, in Fernandez, Gerbeau, Gloria and Vidrascu [37] the Schur complements are computed after linearization. The approach [85] is also based on the Steklov-Poincaré operator on the interface. Solvers using inexact factorizations were considered by Badia, Quaini, and Quarteroni [2]. In Gerbeau and Vidrascu [46] a quasiNewton approach on the fixed point formulation using a finite difference approximation of the Jacobian or reduced models was studied, resulting in Newton-like methods. The Interface Quasi-Newton methods (IQN) was considered in [28, 29].

Monolithic algorithms were investigated, e.g., by Hron and Turek [49], by Küttler, Gee, Förster, Comerford, and Wall [54], by Gee, Küttler, and Wall [45], by Bazilevs et al. [14], by Barker and Cai [13, 84], and by Crosetto, Deparis, Fourestey, and Quarteroni [26].

Parallel algebraic multigrid preconditioners have recently been applied to fully monolithic ALE formulations of FSI problems in the setting of biomechanics, see, e.g., Gee, Küttler, and Wall in [45] and Bazilev et al. [14]. Overlapping Schwarz methods within monolithic approaches were studied in different regimes of severity of the added-mass effect in [26] confirming successful results for 2D obtained already by Barker and Cai [13].

As in this paper, see (11), in [49] and [65] the ALE mapping is obtained as the solution of a Laplace equation but it is also possible to use elasticity [69]. There are alternative approaches to the ALE framework. Among these are XFEM approaches; see [82] and references therein. In [63, 68, 32], a fully Eulerian formulation of FSI is used in 2D, to avoid the degeneration of the ALE mapping and to facilitate adaptivity. Other alternatives are space-time finite element methods, see, e.g., [78, 50, 14], and Eulerian level set formulations; see [25] or [83]. The Immersed Boundary Method can also be applied to FSI problems; see [61]. Comparisons of different time-stepping schemes for FSI problems in ALE-formulation are also known, see, e.g., in Razzaq, Hron, and Turek [65].

Constructing preconditioners for Navier-Stokes equations is challenging by itself. Among the many approaches are physics-based methods such as SIMPLE [60]. Others are based on approximate factorization of the Navier-Stokes equations [62]. The pressure convection-diffusion (PCD) preconditioner is based on a factorization which converges in at most two GMRES iterations; see [58]. Another approximation of the Schur complement leads to the least-squares commutator (LSC) preconditioner [33, 35]. Moreover, Elman et al. [34] present a comparison of the previous preconditioners. Benzi et al. [16, 17] have introduced the augmented Lagrangian (AL) preconditioners which are based on an augmented Lagrangian formulation of the corresponding saddle point problem. A comparison between the PCD, the LSC and the AL approaches has been discussed in [81].

A lot of work on the mechanical interaction of biological surrounding structures with an interior blood flow is focused on the qualitatively correct physiological simulation of the hemodynamics. Here, even simple structural models, e.g., linear elasticity, as in [26], or simple discretizations, e.g., P1, as in [79], can be sufficient.

One of our main objectives, however, is to compute realistic transmural stress distributions resulting from the interior blood flow in an artery. This requires a realistic, i.e., nonlinear and anisotropic model of the wall structure including eigenstresses; see [36]. As a result of the almost incompressibility a suitable discretization is also necessary; it is to be expected that simple P1 finite elements will not be sufficient. Nonlinear Saint Venant-Kirchhoff and Neo-Hooke material laws were applied to FSI of biological tissues by Küttler et al. [54] in different strong coupling schemes. To the best of our knowledge, the first approach using nonlinear, polyconvex, anisotropic structural models in the context of FSI has been considered in [8].

Gee, Förster, and Wall proposed methods to compute prestresses in the isotropic large deformation setting and compared them using a 3D model of an abdominal aortic aneurysm recovered from patientspecific CT geometry data in [44]. They also have reported that FSI simulations lead to unrealistic wall deformations unless the prestress is accounted for. 


\subsection{Monolithic Fluid-Structure Interaction Algorithms}

We give a short, incomplete review on monolithic FSI algorithms. A monolithic FSI approach coupling nonlinear hyperelastic solid models with Navier-Stokes is presented in Hron and Turek [49], considering the incompressible case for the solid. They take a systematic approach starting from 2D; see also Turek et al. [65]. For the solution of the linear saddle point systems a sparse direct solver, an ILU preconditioner, and a geometric multigrid method with a Vanka-type smoother are considered. A block preconditioner with Schur complements for the monolithic system is presented in [52]. A brain aneurysm in 2D is discussed in [80], using Neo-Hooke for the structure.

A scalable monolithic solver for an FSI problem coupling blood flow with a conforming arterial wall in 2D is presented in Barker [12] and Barker and Cai [13]. A Newton scheme with an explicitly computed Jacobian is applied; see also [38], [14], and [13]. For the solution of the arising linearized systems Barker and Cai use a hybrid multilevel Schwarz preconditioner which uses restricted additive Schwarz on the fine level and multiplicative Schwarz on the coarse level. The parallel Newton-Krylov-Schwarz approach for the monolithic system is extended to three dimensions in $\mathrm{Wu}$ and Cai [84], and scalability is shown for up to three thousand processors. The solution approach is related to ours, although we apply Schwarz methods on the blocks of a monolithic Dirichlet-Neumann preconditioner. In [45], Gee, Küttler, and Wall use a monolithic ALE approach to couple a nonlinear Saint-Venant Kirchhoff model with a Navier-Stokes fluid in 3D and solve the arising equations using a Newton scheme based on an exact Jacobian. The authors consider block preconditioners for the monolithic system, i.e., Block-Gauss-Seidel, using AMG for the blocks, as well as a new AMG scheme using Block-Gauss-Seidel smoothing on all levels. The block-AMG approaches have already been compared with partitioned approaches in [54]. Recently, Langer and Yang [56, 55] considered a Dirichlet-Neumann method for fluid-structure interaction problems in biomechanics using a Mooney-Rivlin model and a straight tube. Mayr, Klöppel, Wall, and Gee [57] present a monolithic FSI approach using dual mortars. In our approach, we are able to handle nonmatching grids by using radial basis functions, cf. [31]. In Turek [64], a isotropic Neo-Hookean material model is used to model FSI in an aneurysm, and Q2P1disc finite elements are used.

The remainder of the paper is organized as follows. In Section 2, we introduce the continuum mechanical constitutive framework, and we describe the material models under consideration. In Section 3, we focus on the fluid-structure interaction problem governing the blood flow in compliant arteries. In addition, the monolithic algorithm used to solve the fully discrete FSI problem in a parallel setting is presented. Section 4 deals with the description and numerical simulations on the benchmark problem proposed. We report and discuss the most relevant numerical results obtained. In Appendix 6, we collect all the results obtained in our simulations.

\section{Mechanical Model for the Arterial Tissue}

In this section, the material model for the structure, i.e., the vessel wall, is described. The model is formulated in terms of classical continuum mechanics at finite strains, where the deformation gradient $\mathbf{F}$ and the right Cauchy-Green tensor $\mathbf{C}=\mathbf{F}^{T} \mathbf{F}$ are considered. In order to account for the anisotropy resulting mainly from the reinforcing fibers (collagen and smooth muscle cells) the concept of structural tensors is applied, see, e.g., [18]. In arteries mainly two fiber family directions are arranged cross-wise helically around the wall. By assuming a weak interaction of these two families the standard approach is to consider an additively decoupled energy consisting of an isotropic part $\psi^{\text {isot }}$ for the elastin-rich ground substance and the superposition of two transversely isotropic parts $\psi_{(a)}^{\mathrm{ti}, \infty}$ for the individual fiber family $(a)$ with direction vector $\mathbf{a}_{(a)}$. The isotropic energy is considered to depend on the right Cauchy-Green tensor in order to a priori fulfill the objectivity condition, i.e., $\psi^{\text {isot }}:=\psi^{\text {isot }}(\mathbf{C})$. For the transversely isotropic part, an additional argument tensor, the structural tensor for transverse isotropy $\mathbf{M}_{(a)}=\mathbf{a}_{(a)} \otimes \mathbf{a}_{(a)}$, is considered 
such that $\psi_{(a)}^{\mathrm{ti}, \infty}:=\psi_{(a)}^{\mathrm{ti}, \infty}\left(\mathbf{C}, \mathbf{M}_{(a)}\right)$. In order to arrive at a more convenient construction of specific energy functions a coordinate-invariant representation in terms of the principal and mixed invariants

$$
I_{1}=\operatorname{tr} \mathbf{C}, I_{2}=\operatorname{tr}[\operatorname{Cof} \mathbf{C}], I_{3}=\operatorname{det} \mathbf{C}, J_{4}=\operatorname{tr}[\mathbf{C M}], J_{5}=\operatorname{tr}\left[\mathbf{C}^{2} \mathbf{M}\right]
$$

is considered. For the isotropic part, we focus on a Neo-Hooke-type representation and skip the dependency of $I_{2}$. For the transversely isotropic part note that the fifth invariant is not polyconvex by itself and thus the alternative invariant

$$
K_{3}^{(a)}:=I_{1} J_{4}^{(a)}-J_{5}^{(a)},
$$

is considered, see [71]. Finally, the polynomial basis for the representation of the strain energy for two fiber families reads $\mathcal{P}:=\left\{I_{1}, I_{3}, K_{(1)}^{3}, K_{(2)}^{3}\right\}$ and the structure of the strain energy is

$$
\psi\left(\mathbf{C}, \mathbf{M}_{(1)}, \mathbf{M}_{(2)}\right)=\psi^{\mathrm{isot}}\left(I_{1}, I_{3}\right)+\sum_{a=1}^{2} \psi_{(a)}^{\mathrm{ti}, \infty}\left(I_{1}, K_{3}^{(a)}\right),
$$

cf. [48]. The polyconvexity condition in the sense of [3] is the essential condition to ensure the existence of minimizers and material stability, cf. [72], where the latter aspect is analyzed in terms of an accompanied localization analysis. Therefore, polyconvex energy functions have to be considered and thus, the NeoHooke-type function is considered for the isotropic part and for the transversely isotropic part the function for arterial tissues proposed in [10] is used, see also [9], where this function is also applied in an engineering context. The two parts of the strain energy function are then given by

$$
\psi^{\text {isot }}=\epsilon_{1}\left(I_{3}^{\epsilon_{2}}+I_{3}^{-\epsilon_{2}}-2\right)+c_{1}\left(I_{1} I_{3}^{-1 / 3}-3\right) \quad \text { and } \quad \psi_{(a)}^{t i, \infty}=\alpha_{1}\left\langle K_{3}^{(a)}-2\right\rangle^{\alpha_{2}}
$$

where the restrictions $c_{1}>0, \epsilon_{1}>0, \epsilon_{2}>1, \alpha_{1}>0$ and $\alpha_{2}>2$ ensure polyconvexity and smooth tangent moduli; the Macaulay brackets are defined as $\langle(\bullet)\rangle=1 / 2(|(\bullet)|+(\bullet))$. Note that a volumetric-isochoric split is considered for the isotropic function, but not for the transversely isotropic part in order to avoid the unphysical behavior observed in [70]. The Cauchy stresses can then be computed by $\sigma=(\operatorname{det}[\mathbf{F}])^{-1} \mathbf{F S F}^{T}$, wherein the second Piola-Kirchhoff stresses are obtained by deriving the strain energy function with respect to the right Cauchy-Green tensor, i.e., $\mathbf{S}=2 \partial_{\mathbf{C}} \psi(\mathbf{C})$. To account for the viscoelastic response mainly induced by the smooth-muscle cells, an internal variable $\mathbf{Q}_{\alpha(a)}^{\mathrm{ti}}$ is introduced on the stress level, which is associated with the stresses in the fibers. Thereby, these internal variables characterize the viscoelastic overstress in fiber direction. This is in contrast to the approach in [47] where also the isotropic elastin-rich ground substance is considered to be viscoelastic. Moreover, here the overstress part is not considered to be isochoric in order to avoid an unphysical viscoelastic response which may result otherwise from the same motivation as in [70]. Computing the second Piola-Kirchhoff stresses for each individual part of the strain energy, i.e., $\mathbf{S}^{\text {isot }}:=2 \partial_{\mathbf{C}} \psi^{\text {isot }}$ and $\mathbf{S}_{(a)}^{\mathrm{ti}, \infty}:=2 \partial_{\mathbf{C}} \psi_{(a)}^{\mathrm{ti}, \infty}$ and adding the overstress yields the second Piola-Kirchhoff stresses

$$
\mathbf{S}=\mathbf{S}^{\mathrm{isot}}+\sum_{a=1}^{2}\left[\mathbf{S}_{(a)}^{\mathrm{ti}, \infty}+\sum_{\alpha=1}^{m} \mathbf{Q}_{\alpha(a)}^{\mathrm{ti}}\right],
$$

where the inner summation represents the classical parallel arrangement of viscoelastic elements, cf. [73]. For the evolution of the overstress the linear differential equation

$$
\dot{\mathbf{Q}}_{\alpha(a)}^{\mathrm{ti}}+\frac{\mathbf{Q}_{\alpha(a)}^{\mathrm{ti}}}{\tau_{\alpha}}=\beta_{\alpha}^{\infty} \dot{\mathbf{S}}_{(a)}^{\mathrm{ti}, \infty},
$$

has to be solved. Herein, the viscoelastic material parameters are the relaxation time parameter $\tau_{\alpha}$ and the associated viscoelastic intensity $\beta_{\alpha}$. The differential equation is solved by means of a convolution integral 
using the midpoint-rule such that the update formula

$$
\mathbf{Q}_{\alpha(a)}^{t i}=\mathbf{H}_{\alpha(a) n}+\hat{\mathbf{Q}}_{\alpha(a)}^{t i}
$$

is obtained. Herein, $\mathbf{H}_{\alpha(a) n}$ solely depends on quantities evaluated at the last time step indexed by $n$, which is given by

$$
\mathbf{H}_{\alpha(a) n}=\exp \left(\frac{-\Delta t}{2 \tau_{\alpha}}\right)\left[\exp \left(\frac{-\Delta t}{2 \tau_{\alpha}}\right)\left\{\mathbf{Q}_{\alpha(a)}^{\mathrm{ti}}\right\}_{n}-\beta_{\alpha}^{\infty}\left\{\mathbf{S}_{(a)}^{\mathrm{ti}, \infty}\right\}_{n}\right]
$$

with $\Delta t$ denoting the time increment. The second additive part in (7) is governed by the hyperelastic component of the transversely isotropic second Piola-Kirchoff stresses from the current time step. It is computed by

$$
\hat{\mathbf{Q}}_{\alpha(a)}^{\mathrm{ti}}=\beta_{\alpha}^{\infty} \exp \left(\frac{-\Delta t}{2 \tau_{\alpha}}\right) \mathbf{S}_{(a)}^{\mathrm{ti}, \infty},
$$

wherein the index for the current time step is skipped. For further information regarding the derivation of the update formulae from the evolution equation the reader is referred to [47] and the references therein. Here, only one viscoelastic relaxation process, i.e. $m=1$, is considered in order to keep the number of additional material parameters small. For the implementation of this model in a finite element program the tangent moduli, i.e., the derivatives of the second Piola-Kirchhoff stresses with respect to the right Cauchy-Green tensor are required. Here, these are numerically computed using the complex-step derivative approximation scheme which is proposed for the calculation of tangent moduli in a finite strain setting in $[76]$.
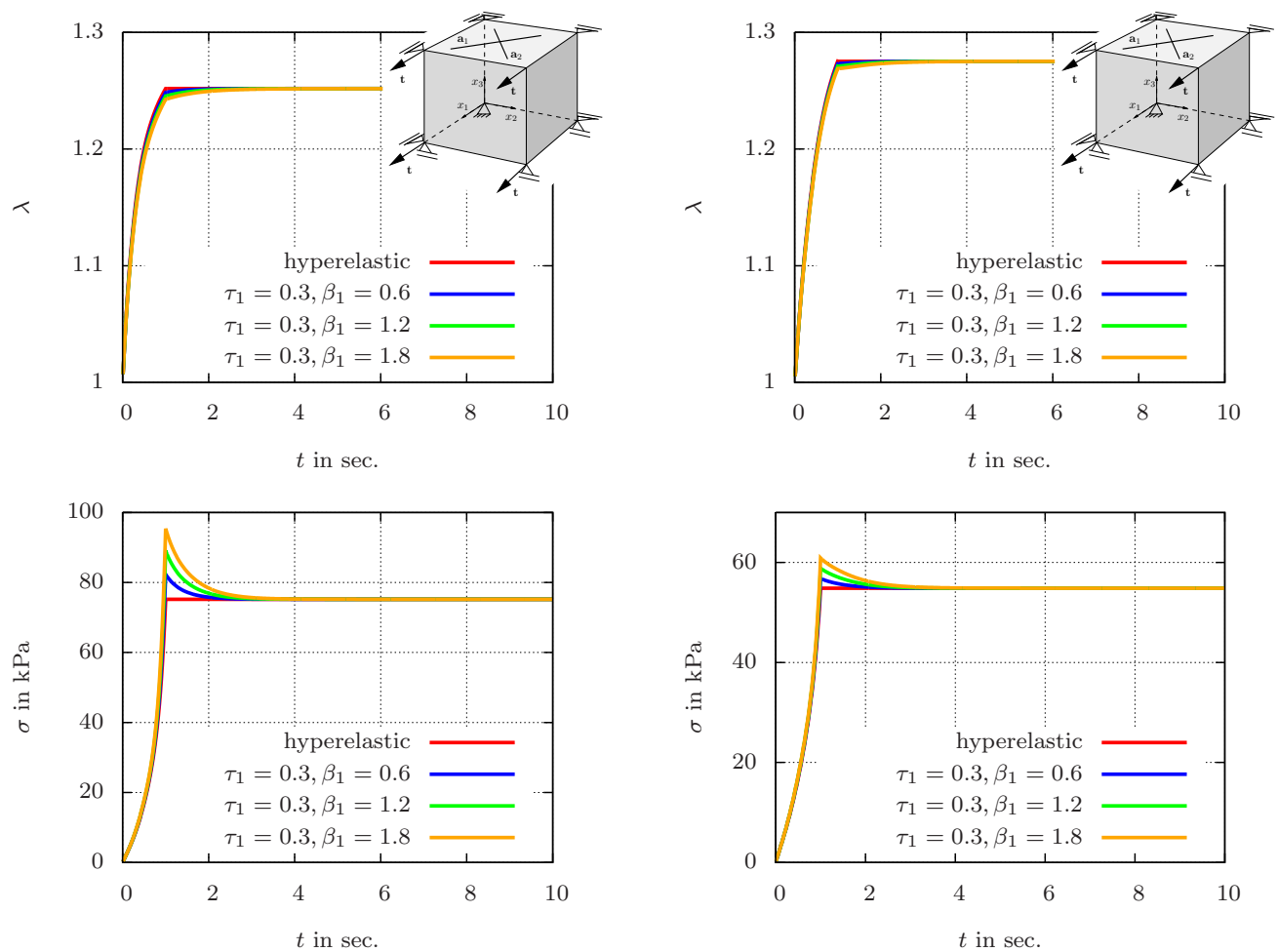

Fig. 1 Creep (top) and relaxation (bottom) tests in circumferential (left) and axial (right) direction for different viscoelastic material parameter sets. 
To illustrate the model response, virtual creep and relaxation tests are performed for different viscoelastic parameters $\beta_{1}$ and $\tau_{1}$ in circumferential as well as in axial direction. The hyperelastic parameters $c_{1}, \epsilon_{1}, \epsilon_{2}$, $\alpha_{1}$ and $\alpha_{2}$ are taken from [20], where they have been adjusted to experimental data of a media of a human artery with the side constraint that an improved robustness of numerical computations can be achieved. For the creep tests a stress of $75 \mathrm{kPa}$ is applied incrementally in steps of one second in circumferential direction and $55 \mathrm{kPa}$ in axial direction. Then, the stresses are kept constant for 9 further seconds in order to analyze the resulting creep behavior. The values for the stresses applied were obtained from a simplified numerical computation where only a hyperelastic solid structure of an axial straight segment of an arterial wall was loaded by an internal hydrostatic pressure of $80 \mathrm{mmHg}$. Then the average circumferential and axial stresses over a part of the wall thickness, where higher stress values were found, were computed. These are assumed to correlate with stresses found in a arterial wall under blood pressure. For the relaxation tests a stretch of $\lambda=1.25$ in circumferential and $\lambda=1.27$ in axial direction is applied within one second and then again kept constant for further 9 seconds. These stretches are associated to the stresses considered in the creep tests. The fiber angle (angle between the circumferential and the fiber direction in an arterial wall) is set to $\bar{\beta}_{\mathrm{f}}=43^{\circ}$. The results obtained are shown in Figure 1 and illustrate the influence of the viscoelastic intensity parameters $\beta_{\alpha}$ as well as the anisotropic behavior of the material by comparing the results for circumferential and axial direction. It is found that the sensitivity to the parameters is higher in the relaxation tests compared to the creep tests. The parameters considered lead to relatively large overstresses which cannot be expected in arterial tissues. They may still enable an analysis with respect to the sensitivity of viscoelasticity in arteries. In numerical simulations of arteries the influence is however not expected to be very large because arteries are mainly deformed by the blood flow and less by applied displacements, which rather corresponds to the creep test, where the sensitivity is comparatively small.

\section{Fluid-Structure Interaction}

\subsection{Model Description}

In this section, we introduce the fluid-structure interaction problem. Let $\Omega^{f}$ and $\Omega^{s}$ be the domains occupied by the fluid and the solid, respectively, in their reference configuration. We denote by $\Gamma=$ $\partial \Omega^{f} \cap \partial \Omega^{s}$ the fluid-structure interface on the reference configuration. At any time $t>0$, the domain occupied by the fluid $\Omega_{t}^{f}$ can be retrieved from $\Omega^{f}$ by the Arbitrary Lagrangian Eulerian (ALE) mapping

$$
\begin{aligned}
\mathcal{A}_{t}: & \Omega^{f} \rightarrow \Omega_{t}^{f} \\
\mathbf{X} & \mapsto \mathcal{A}_{t}(\mathbf{X})=\mathbf{X}+\mathbf{d}_{f}(\mathbf{X}),
\end{aligned}
$$

where $\mathbf{d}_{f}$ represents the displacement of the fluid domain. The use of the ALE formulation allows an arbitrary reconstruction of the volumetric finite element grid in $\Omega_{t}^{f}$ from the one on the domain boundary $\partial \Omega_{t}^{f}$. For the sake of computation, this reconstruction is directly operated on the reference configuration by means of a harmonic extension of the structure displacement $\mathbf{d}_{s}$ at the fluid-structure interface $\Gamma$ to the interior of the reference fluid domain $\Omega^{f}$, i.e.,

$$
\left\{\begin{array}{l}
-\Delta \mathbf{d}_{f}=\mathbf{0} \text { in } \Omega^{f}, \\
\mathbf{d}_{f}=\mathbf{d}_{s} \text { on } \Gamma, \\
\mathbf{d}_{f} \cdot \mathbf{n}_{f}=0 \text { on } \partial \Omega^{f} \backslash \Gamma,
\end{array}\right.
$$

where $\mathbf{n}_{f}$ is the outward unit normal to the reference fluid domain boundary. Since the structure displacement $\mathbf{d}_{s}$ changes in time, the harmonic extension (11) allows to define the current configuration of the fluid domain $\Omega_{t}^{f}=\mathcal{A}_{t}\left(\Omega^{f}\right)$ thanks to the ALE map parametrization (10). 
In our fluid-structure interaction model, we consider the fluid dynamics governed by the incompressible Navier-Stokes equations written in the ALE frame of reference [15, 43],

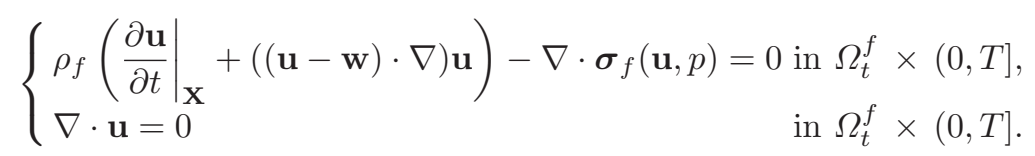

In (12), $\left.\frac{\partial}{\partial t}\right|_{\mathbf{X}}=\frac{\partial}{\partial t}+\mathbf{w} \cdot \nabla$ is the ALE derivative, $(0, T]$ is the time interval, $\rho_{f}$ is the fluid density, $\mathbf{u}$ and $p$ are the fluid velocity and pressure, respectively, and $\boldsymbol{\sigma}_{f}(\mathbf{u}, p)=2 \mu_{f} \boldsymbol{\epsilon}(\mathbf{u})-p \mathbf{I}$ is the Cauchy stress tensor (I is the identity matrix). We denoted by $\boldsymbol{\epsilon}(\mathbf{u})=\frac{1}{2}\left(\nabla \mathbf{u}+(\nabla \mathbf{u})^{T}\right)$ the strain rate tensor and by $\mu_{f}$ the dynamic viscosity of the fluid. Furthermore, $\mathbf{w}$ is the fluid mesh velocity,

$$
\mathbf{w}=\left.\frac{\partial \mathbf{d}_{f}}{\partial t}\right|_{\mathbf{X}} .
$$

We formulate the structure problem in a purely Lagrangian frame of reference. The conservation of momentum for the structure reads

$$
\rho_{s} \frac{\partial^{2} \mathbf{d}_{s}}{\partial t^{2}}-\nabla \cdot(\mathbf{F S})=0 \quad \text { in } \Omega^{s} \times(0, T]
$$

where $\rho_{s}$ is the density of the structure and $\mathbf{F S}$ are the first Piola-Kirchhoff stresses.

The coupling between the geometry, fluid and structure subproblems is expressed by

$$
\begin{aligned}
\mathbf{d}_{f} & =\mathbf{d}_{s}, \\
\frac{\partial \mathbf{d}_{s}}{\partial t} & =\mathbf{u} \circ \mathcal{A}_{t}, \\
(\operatorname{det}[\mathbf{F}])^{-1} \mathbf{F}^{-T} \boldsymbol{\sigma}_{f} \mathbf{n}_{f}+(\mathbf{F S}) \mathbf{n}_{s} & =0,
\end{aligned}
$$

where (14) expresses the geometric adherence, (15) the continuity of the velocities (kinematic condition) and (16) the continuity of the stresses (dynamic condition) on $\Gamma$.

The resulting system of equations describing the FSI problem is nonlinear due to the moving fluid domain, the convective term in the fluid momentum equation, and the possible nonlinearity of the structural material model. In this publication, we use indeed highly nonlinear structural material models formulated in a finite strain framework.

\subsection{Monolithic Algorithm for Fluid-Structure Interaction}

We use finite differences for the approximation of the time derivatives of both the fluid and the structure equations, and the finite element method for the space discretization. We choose conforming fluid and structure meshes at the interface. Specifically, in this work we consider three different space discretizations of the FSI problem that we will refer to as "P1", "P2", and "F"; see Table 1. After space discretization, a monolithic Convective Explicit (CE) algorithm is employed to numerically solve the fully coupled FSI problem $[2,26]$. The structure and the geometry subproblems are treated fully implicitly, while the convective term of the fluid momentum is linearized as follows:

$$
\left(\left(\mathbf{u}^{n+1}-\mathbf{w}^{n+1}\right) \cdot \nabla\right) \mathbf{u}^{n+1} \approx\left(\left(\mathbf{u}^{*}-\mathbf{w}^{*}\right) \cdot \nabla\right) \mathbf{u}^{n+1} .
$$


In (17), $\mathbf{u}^{*}$ and $\mathbf{w}^{*}$ represent temporal extrapolations of the fluid velocity and of the fluid domain velocity, respectively. This choice is suitable when the Reynolds number that characterizes the fluid flow is not high (condition typically fulfilled in the problem at hand).

After space-time discretization, the fully coupled nonlinear FSI system reads

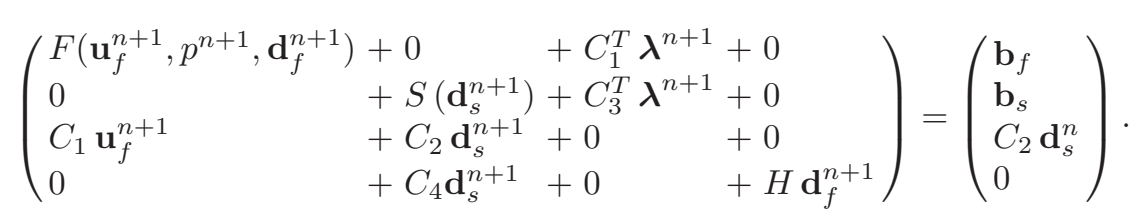

We denote by $\boldsymbol{\lambda}$ the vector containing the Lagrange multipliers that are used to enforce the balance of normal stresses across $\Gamma$. In system (18), the fluid subproblem $F$ is nonlinear due to the moving fluid domain, the solid subproblem $S$ is nonlinear due to the material law and finite strain setting used to model the structure deformations, while the geometry subproblem $H$ is linear. The matrices $C_{1}$ and $C_{2}$ account for the continuity of the velocity on $\Gamma$, the transposed matrices $C_{1}^{T}$ and $C_{3}^{T}$ account for the balance of normal stresses (imposed weakly), whereas $C_{4}$ accounts for the geometric adherence. Dealing with conforming meshes and conforming discretizations at the fluid-structure interface yields: $\left.C_{1}\right|_{\Gamma}=$ $\left.I\right|_{\Gamma},\left.C_{3}\right|_{\Gamma}=-\left.I\right|_{\Gamma},\left.C_{2}\right|_{\Gamma}=1 / \Delta t C_{3},\left.C_{4}\right|_{\Gamma}=\left.I\right|_{\Gamma}$ where $\left.I\right|_{\Gamma}$ is the identity matrix defined on the interface $\Gamma$.

\subsection{Three-field Approach for the Structure}

For the structure, we will use the " $\overline{\mathrm{F}}$ "-approach, a three-field (mixed) formulation that was introduced to avoid volumetric locking effects in the finite element solution from the almost incompressibility; see Simo $[74$, Section 45$]$. Let $J=J(\varphi)=\operatorname{det}(\mathbf{F})$, then we have

$$
\mathbf{F}=J^{1 / 3} \tilde{\mathbf{F}}, \quad \tilde{\mathbf{F}}=J^{-1 / 3} \mathbf{F} .
$$

We introduce a new scalar variable $\theta$, satisfying $\theta=J$ in a weak sense, and we define

$$
\overline{\mathbf{F}}:=\theta^{1 / 3} \tilde{\mathbf{F}}, \quad \overline{\mathbf{C}}:=\overline{\mathbf{F}}^{T} \overline{\mathbf{F}}
$$

with $\overline{\mathbf{F}}=\overline{\mathbf{F}}(\boldsymbol{\varphi}, \theta), \overline{\mathbf{C}}=\overline{\mathbf{C}}(\boldsymbol{\varphi}, \theta)$. We consider the following three-field Lagrangian

$$
\mathrm{E}(\boldsymbol{\varphi}, \theta, \pi)=\int_{\Omega} W(\overline{\mathbf{C}}(\boldsymbol{\varphi}, \theta))+\pi(J(\boldsymbol{\varphi})-\theta) d x-V_{\mathrm{ext}}(\boldsymbol{\varphi}),
$$

where $V_{\text {ext }}(\varphi)$ is the potential energy of external forces; for more details, see Simo [74, Section 45]. We use a $P_{2}-P_{0}-P_{0}$ mixed finite element discretization, i.e., piecewise quadratic elements for the deformation field $\varphi$ and piecewise constant elements for the scalar fields $\theta$ and $\pi$. Local static condensation of $\theta$ and $\pi$ on each finite element leads to a reduced problem which is then solved.

As a result of the $\overline{\mathrm{F}}$-discretization volumetric changes are not penalized point-wise but rather in an element-wise average sense by the term $\epsilon_{1}\left(I_{3}^{\epsilon_{2}}+I_{3}^{-\epsilon_{2}}\right)$; see the hyperelastic energy in (4). Let us note here that, since the almost incompressibility constraint is nonlinear, there is no direct relation to a Poisson's ratio in the linear case. The severity of potential locking, if standard finite elements are used, is therefore difficult to assess a priori. Typical parameter sets for biological soft tissue can still result in a volumetric change at the order of one percent [21], which is considered acceptable. This is one of the reasons of our numerical study with respect to the space discretization of the arterial wall; see, e.g., Section 4.2.2 and Section 4.3. We believe that our numerical results indeed show that, to obtain good estimates of all quantities of interest, P1 finite elements are not sufficient even for simulations within the physiological 
range; see Section 4.5 on the stresses. Surprisingly, for viscoelasticity, we even observe a qualitatively wrong behavior using P1 elements; see Section 4.2.4.

The implementation of the almost incompressibility constraint by a penalty can pose challenges to direct and iterative solvers for the linearized systems. In [21, 19], it was observed that an augmented Lagrange approach can be profitable computationally in the context of soft biological tissue: In the quasi-static setting larger pseudo-time steps could be chosen and the number of Krylov iteration for the linearized systems was reduced. It is also noteworthy that, using augmented Lagrange, the element-wise volumetric change can exactly be controlled, whereas, using a penalty, the violation is known only a-posteriori. In this paper, however, to avoid additional complications, we do not apply an augmented Lagrange approach for the incompressibility. This increases the challenges for the iterative solution method, however.

\begin{tabular}{|c|c|c|c|}
\hline Short Name & Fluid (velocity-pressure) & Structure & Geometry \\
\hline "P1" & P1-P1 stabilized & P1 & P1 \\
"P2" & P2-P1 & P2 & P2 \\
"F" & P2-P1 & $\bar{F}$ & P2 \\
\hline
\end{tabular}

Table 1 Description of the space discretizations considered.

We remark that when using the "P1"-approach, the fluid velocity and pressure are discretized by P1-P1 finite elements stabilized by interior penalty [22]. Finally, although the choice of the discretizations "P2" and " $\overline{\mathrm{F}} "$ would lead to isoparametric meshes, we keep straight tetrahedral elements in our computational grids.

\subsection{Linearization and Parallel Preconditioner}

We solve the nonlinear problem (18) by means of the Newton method. At each time step, the Newton algorithm yields of the following linear system

$$
J_{\mathrm{CE}}\left(\mathbf{x}_{k}^{n+1}\right) \boldsymbol{\delta}_{k+1}=-\mathbf{r}\left(\mathbf{x}_{k}^{n+1}\right),
$$

where we denote by $k$ the index of the Newton iterations, by $J_{\mathrm{CE}}\left(\mathbf{x}_{k}^{n+1}\right)$ the Jacobian matrix associated to the linearized FSI problem, by $\mathbf{r}\left(\mathbf{x}_{k}^{n+1}\right)$ the residual, by $\boldsymbol{\delta}_{k+1}$ the Newton increment, and $\mathbf{x}_{k}^{n+1}=$ $\left(\mathbf{u}, p, \mathbf{d}_{s}, \boldsymbol{\lambda}, \mathbf{d}_{f}\right)$ is the solution vector.

The Jacobian matrix associated to the FSI problem reads

$$
J_{C E}=\left(\begin{array}{cccc}
F & 0 & C_{1}^{T} & D_{\mathbf{d}_{f}} F \\
0 & D_{\mathbf{d}_{s}} S & C_{3}^{T} & 0 \\
C_{1} & C_{2} & 0 & 0 \\
0 & C_{4} & 0 & H
\end{array}\right)
$$

where $D_{\mathbf{d}_{f}} F$ denotes the shape derivatives, cf. [39], and $D_{\mathbf{d}_{s}} S$ the linearization of the solid part.

For each $k$, we solve (19) using the GMRES method preconditioned by an approximated monolithic Dirichlet-Neumann preconditioner $P_{D N}$, cf. [26, 27]. The latter $P_{D N}$ is constructed from $J_{C E}$ by neglecting the coupling block $C_{3}^{T}$ and then applying a block factorization to $J_{C E}$. The result is:

$$
P_{D N}=\left(\begin{array}{cccc}
I & 0 & 0 & 0 \\
0 & D_{\mathbf{d}_{s}} S & 0 & 0 \\
0 & 0 & I & 0 \\
0 & 0 & 0 & I
\end{array}\right)\left(\begin{array}{cccc}
I & 0 & 0 & 0 \\
0 & I & 0 & 0 \\
0 & 0 & I & 0 \\
0 & C_{4} & 0 & H
\end{array}\right)\left(\begin{array}{cccc}
F & 0 & C_{1}^{T} & D_{\mathbf{d}_{f}} F \\
0 & I & 0 & 0 \\
C_{1} & C_{2} & 0 & 0 \\
0 & 0 & 0 & I
\end{array}\right)
$$


The inverse of each factor of $P_{D N}$ is approximated by one level algebraic additive Schwarz preconditioners, cf. [75].

\section{Numerical Experiments on Fluid-Structure Interaction}

In this section, we introduce the benchmark problem, a curved pipe mimicking a tract of an artery, which is the basis of our simulations. It includes an initialization ("ramp") phase and a heartbeat phase. The ramp phase is needed in order to inflate the curved geometry, and the second phase includes the periodic application of the inflow profile of a human heartbeat. First, we will provide detailed information about the geometry and the corresponding meshes, the material parameters and the boundary conditions of our boundary value problem, and then add some remarks about the software which has been used for our simulations. Finally, we will present a detailed discussion of the results, including mesh convergence studies, investigation of the boundary conditions, and comparisons of space discretizations and material models. If not stated otherwise, we choose an absolute tolerance of $10^{-7}$ as a stopping criterion for the Newton method, and a relative tolerance of $10^{-8}$ for the GMRES iterations.

The complete set of our computational results can be found in Appendix 6, whereas in this section, we will just present those results relevant to our discussion.

\subsection{Settings of the Simulations}

In order to be able to interpret the results, we define an idealized geometry and simplified boundary conditions, which still show characteristic results. As we focus on the numerical analysis of sophisticated arterial wall models in this publication, the boundary value problem is constructed such that effects due to nonlinearities are revealed, and the importance of the use of such material models in general applications is highlighted. We also describe the temporal and spatial discretizations used in our simulations. Finally, we briefly mention the software packages used to carry out our simulations.

\subsubsection{Geometry}

In Figure 2, the geometry and its dimensions are shown. It consists of a curved and a straight section and can be seen as an idealized coronary artery. We restrict ourselves to just one material, i.e., the media, whereas the wall thickness is chosen according to realistic arteries.

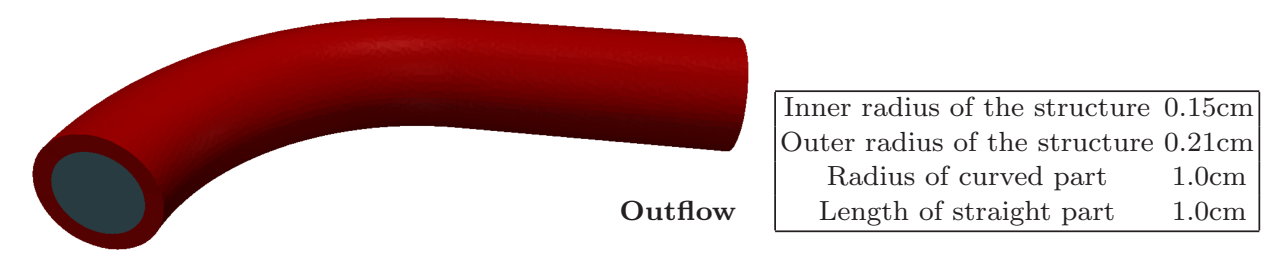

Inflow

Fig. 2 Geometry of the FSI problem. 


\subsubsection{Material Parameters}

For the modeling of the media, we use the hyperelastic and the viscoelastic material models which are described in Section 2. We choose the material parameters from [20, 5, ( $\Psi_{A}$ Set 2)], cf. Table 2, for the hyperelastic material model. These parameters are fitted to the material response of the media of a real artery. For the viscoelastic material, we use two different parameter sets, cf. Table 3, where Set 2 in Table 3 has a significantly reduced relaxation time in order to show viscoelastic effects more clearly.

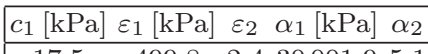

$\begin{array}{llllll}17.5 & 499.8 & 2.4 & 30001.9 & 5.1\end{array}$

Table 2 Parameters for the hyperelastic material model; see Section 2.

\begin{tabular}{|c|ccccccc|}
\hline Set & $c_{1}[\mathrm{kPa}]$ & $\varepsilon_{1}[\mathrm{kPa}]$ & $\varepsilon_{2}$ & $\alpha_{1}[\mathrm{kPa}]$ & $\alpha_{2}$ & $\tau_{1}$ & $\beta_{1}$ \\
\hline 1 & 17.5 & 499.8 & 2.4 & 30001.9 & 5.1 & 2.0 & 1.0 \\
2 & 17.5 & 499.8 & 2.4 & 30001.9 & 5.1 & 0.3 & 1.8 \\
\hline
\end{tabular}

Table 3 Parameters for the viscoelastic material model: long relaxation time (Set 1), and short relaxation time (Set 2); see Section 2.

\subsubsection{Time Discretization}

As already mentioned in Section 3.2, for all simulations we use the Convective Explicit time discretization scheme $[26,27]$ while the temporal derivatives are approximated using a second order BDF-scheme. The time discretization scheme and the composed Dirichlet-Neumann preconditioner are described in Sections 3.2 and 3.4. Due to the semi-explicit treatment of the convective term and the fast dynamics of our solution, in general we have to use very fine time steps. We will thus first use a time step $\Delta t=10^{-4} \mathrm{~s}$. As we are going to discuss later, however, it is possible to use larger time steps in the heartbeat phase of the simulation; see Section 4.3.

\subsubsection{Meshes}

\begin{tabular}{|c|c|c|c|c|c|}
\hline Mesh & $\begin{array}{r}\text { \#Fluid } \\
\text { elements }\end{array}$ & $\begin{array}{r}\text { \#Structural } \\
\text { elements }\end{array}$ & $\begin{array}{r}\text { Total } \\
\text { Dofs "P1" }\end{array}$ & $\begin{array}{r}\text { Total } \\
\text { Dofs "P2" }\end{array}$ & $\begin{array}{r}\text { Total } \\
\text { Dofs " } \overline{\mathrm{F}} "\end{array}$ \\
\hline$\# 0$ & 2404 & 12348 & & 96285 & 96285 \\
\hline \#1 & 6549 & 21636 & 30880 & 186658 & 186658 \\
\hline \#2 & 8187 & 45360 & 47995 & 307579 & 307579 \\
\hline \#3 & 12670 & 98742 & 88670 & 590555 & 590555 \\
\hline \#4 & 19978 & 183420 & 146817 & 1016913 & 1016913 \\
\hline \#5 & 40011 & 274500 & 230713 & & - \\
\hline \#6 & 78318 & 517464 & 423534 & - & - \\
\hline$\# 7$ & 179513 & 1036800 & 871323 & & - \\
\hline
\end{tabular}

Table 4 Degrees of freedom of the meshes used in the simulations. The internal variables in $\overline{\mathrm{F}}$ are condensed locally and thus not counted here.

In order to investigate if the computed quantities converge for the different spatial discretizations listed in Section 3.2, cf. Table 1, eight different meshes will be used; cf. Table 4. For P1 elements, these are the 


\begin{tabular}{|r|r|r|r|r|r|}
\hline Mesh & Dofs $\mathbf{u}$ & Dofs $p$ & Dofs $\mathbf{d}_{s}$ & Dofs $\boldsymbol{\lambda}$ & Dofs $\mathbf{d}_{f}$ \\
\hline$\# 1$ & 5430 & 1810 & 14664 & 3546 & 5430 \\
$\# 2$ & 6807 & 2269 & 27648 & 4464 & 6807 \\
$\# 3$ & 10545 & 3515 & 57096 & 6969 & 10545 \\
$\# 4$ & 15345 & 5115 & 101937 & 9075 & 15345 \\
$\# 5$ & 27777 & 9259 & 152295 & 13605 & 27777 \\
$\# 6$ & 51408 & 17136 & 282165 & 21417 & 51408 \\
$\# 7$ & 113175 & 37725 & 564252 & 42996 & 113175 \\
\hline
\end{tabular}

Table 5 Degrees of freedom of the P1 meshes: fluid velocity $(\mathbf{u})$, fluid pressure $(p)$, structural displacement $\left(\mathbf{d}_{s}\right)$, coupling $(\boldsymbol{\lambda})$, and geometry/fluid mesh motion $\left(\mathbf{d}_{f}\right)$.

\begin{tabular}{|r|r|r|r|r|r|}
\hline Mesh & Dofs $\mathbf{u}$ & Dofs $p$ & Dofs $\mathbf{d}_{s}$ & Dofs $\boldsymbol{\lambda}$ & Dofs $\mathbf{d}_{f}$ \\
\hline$\# 0$ & 14505 & 843 & 58296 & 8136 & 14505 \\
$\# 1$ & 34368 & 1810 & 101808 & 14304 & 34368 \\
$\# 2$ & 43071 & 2269 & 201168 & 18000 & 43071 \\
$\# 3$ & 66648 & 3515 & 425700 & 28044 & 66648 \\
$\# 4$ & 100455 & 5115 & 774396 & 36492 & 100455 \\
\hline
\end{tabular}

Table 6 Degrees of freedom of the P2 and $\overline{\mathrm{F}}$ meshes: fluid velocity (u), fluid pressure $(p)$, structural displacement $\left(\mathbf{d}_{s}\right)$, coupling $(\boldsymbol{\lambda})$, and geometry/fluid mesh motion $\left(\mathbf{d}_{f}\right)$. The internal degrees of freedom in the $\overline{\mathrm{F}}$ approach are statically condensated and are thus not considered.

the Meshes \#1 to \#7, for P2 and $\overline{\mathrm{F}}$ elements, the Meshes \#0 to \#4 are sufficient since Mesh \#4 is already fine. For a detailed summary of the degrees of freedom (dofs), cf. Table 5 and 6.

Note that, if we are only interested in fluid quantities, P1 elements for the structure can suffice if a comparatively high number of degrees of freedom is considered, whereas P2, or, even better, $\overline{\mathrm{F}}$ elements, for the structure are necessary for an accurate analysis of the structural stress distributions at a lower number of degrees of freedom.

\subsubsection{Boundary Conditions}

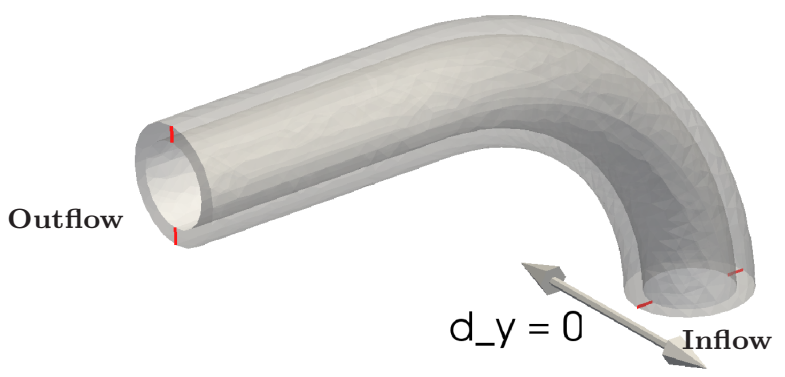

Fig. 3 Dirichlet boundary condition at the inlet and outlet: fixed $y$-displacement for the red colored nodes.

Due to the fact that we simulate a section of a coronary artery without any surrounding tissue, appropriate boundary conditions have to be chosen in order to replace the circulatory system and to statically determine the artery. The structure is fixed in the directions perpendicular to the respective faces at the inlet and outlet. Additionally, we impose zero displacement in $y$-direction for all nodes at the inlet and outlet of the structure with $y=0$; cf. the red lines in Figure 3.

The inflow boundary condition for the fluid is of particular importance, because it is the driving force for our FSI simulation. In the ramp phase, we apply an increasing inflow flow rate. Using this, together with an absorbing type boundary condition [59], allows to obtain a steady condition at the end of the ramp phase, for which the internal blood pressure is $p_{\text {steady }}=80 \mathrm{mmHg}$ and the flow rate is $Q_{\text {steady }}=3 \mathrm{~cm}^{3} / \mathrm{s}$; 
see Section 4.2. In that way, a prestretch of the arterial wall is generated, which is necessary as a starting configuration for a subsequent simulation of realistic heartbeats.

We call $T_{\mathrm{R}}$ the duration until the inflow flow rate reaches its peak value of $Q_{\text {steady }}$, and $T_{\text {steady }}$ the time when the steady state is reached. There are many possible choices for the ramp function $Q_{\text {ramp }}$ to increase the flow rate. They should, of course, satisfy the following conditions:

- $Q_{\text {ramp }}(0 s)=0.0 \mathrm{~cm}^{3} / \mathrm{s}$,

- $Q_{\mathrm{ramp}}(t)=3.0 \mathrm{~cm}^{3} / \mathrm{s} \quad \forall T_{\mathrm{R}} \leq t \leq T_{\text {steady }}$.

The time $T_{\text {steady }}$ strongly depends on the special choice of $T_{\mathrm{R}}$ and $Q_{\mathrm{ramp}}$. In Sections 4.2 .1 and 4.2 .2 we will consider a linear- and a cosine-type ramp to model $Q_{\text {ramp }}$.

In both parts of the simulation we impose the inflow flow rate as a Dirichlet boundary condition for the fluid, i.e., we impose an inflow flow rate $Q_{\text {ramp }}$ over time for the ramp phase and $Q_{\text {heartbeat }}$ for the heartbeat phase, respectively. At each time instance $t^{n+1}$, the value of $Q\left(t^{n+1}\right)=Q^{n+1}$ (we understand by $Q$ either $Q_{\text {ramp }}$ or $Q_{\text {heartbeat }}$ ) is imposed at the inflow section of the fluid domain $\Gamma_{t, i n}^{f}$ by a Dirichlet boundary condition on the velocity, namely $\left.\mathbf{u}^{n+1}(\mathbf{x})\right|_{\Gamma_{t, i n}^{f}}=\left(0,0, u_{z}(\mathbf{x})\right)$ with

$$
u_{z}^{n+1}(\mathbf{x})=\alpha^{n+1} \hat{u}_{z} \circ \mathcal{A}_{t}^{-1}(\mathbf{x})
$$

where

$$
\hat{u}_{z}(\hat{\mathbf{x}})=\frac{R^{2}-\left\|\hat{\mathbf{x}}-\widehat{\mathbf{x}}_{c}\right\|^{2}}{R^{2}}
$$

$\hat{u}_{z}$ is a parabolic profile defined on $\widehat{\Gamma}_{i n}^{f}$, which is the inflow section of the fluid domain at time $t=0$ s. $R$ and $\widehat{\mathbf{x}}_{c}$ are the radius and the barycenter of $\widehat{\Gamma}_{i n}^{f}$, respectively, while $\alpha^{n+1}$ reads

$$
\alpha^{n+1}=\frac{Q^{n+1}}{\widehat{Q}^{n+1}}, \quad \text { being } \quad \widehat{Q}^{n+1}=\int_{\Gamma_{t, i n}^{f}} \hat{u}_{z} \circ \mathcal{A}_{t}^{-1}(\mathbf{x}) \cdot \mathbf{n}_{f} d \gamma
$$

Indeed, this choice ensures that

$$
\int_{\Gamma_{t, i n}^{f}} \mathbf{u}^{n+1} \cdot \mathbf{n}=Q^{n+1} .
$$

Our geometry only represents a section of an idealized coronary artery, thus to properly model the solution's behavior at the artery outflow, an absorbing boundary condition is imposed at the outlet of the fluid. The absorbing boundary condition internally builds on a lower dimensional linear elastic material model. In our nonlinear setting, in general, we can therefore not expect this absorbing boundary condition to completely remove reflections. In the ramp phase, in order to reach at steady state a desired physiological pressure in the fluid, we consider a modified version of the absorbing boundary condition proposed in [59]. At time $t^{n+1}$, the following absorbing boundary condition is used:

$$
\left.\boldsymbol{\sigma}_{f}^{n+1} \cdot \mathbf{n}_{f}\right|_{\Gamma_{\text {out }}}=\left.p_{\text {out }}^{n+1} \mathbf{n}_{f}\right|_{\Gamma_{\text {out }}}
$$

with

$$
p_{\text {out }}^{n+1}=\left(\frac{\sqrt{\rho_{F}}}{2 \sqrt{2}} \frac{Q_{\text {out }}^{n}}{\bar{A}}+\sqrt{\frac{t E}{1-\nu^{2}} \frac{\pi}{A^{0}} \sqrt{A^{0}}}\right)^{2}-\frac{t E}{1-\nu^{2}} \frac{\pi}{A^{0}} \sqrt{A^{0}}+p_{\text {ref }} .
$$

In (27), $E, \nu$ and $t$ are Young's modulus, Poisson's ratio and the thickness of the structure, respectively, $P_{\text {ref }}$ is a reference fluid pressure, $Q_{\text {out }}^{n}$ is the outflow flow rate at the discrete time $t^{n}$, while $A^{0}$ is the area of the outflow section of the fluid domain in its reference configuration.

In our setting, $\bar{A}$ is computed from $(27)$ by imposing the steady state conditions, i.e., $p_{\text {out }}^{n+1}=p_{\text {steady }}=$ $80 \mathrm{mmHg}$ when $Q_{\text {out }}=Q_{\text {steady }}=3 \mathrm{~cm}^{3} / \mathrm{s}$. Note that during the simulations of the heartbeats, the absorbing boundary condition (27) is modified according to [59], i.e. $A_{\text {out }}^{n}$ is used instead of $\bar{A}$. 
We remark that the values of Young's modulus $E$ and Poisson's ratio $\nu$ are adjusted to the response of the nonlinear anisotropic hyperelastic material model described in Section 2. Here, only the material behavior in circumferential direction is considered for the adjustment of the linear elastic absorbing boundary condition, resulting in a Young's modulus of $120 \mathrm{kPa}$ and a Poisson's ratio of 0.49; cf. also Section 4.2.5.

Although an absorbing boundary condition is used at the fluid outflow, from the results shown in Section 4.2.6, we are inclined to believe that the minor oscillations that remain, see, e.g., Section 4.2 .3 and Figure 10, are physical, in the sense that they are simply an artifact of the somewhat frugal boundary conditions for the structure, rather than being an artifact caused by the imperfectly absorbed waves at the outflow. We will discuss the details in Section 4.2.6.

These oscillations vanish over time, cf., e.g., Section 4.2.3. We will consider ramps of different shapes, see Sections 4.2.1 and 4.2.2, and slopes, see Section 4.2.3, to further reduce oscillations. After reaching the steady state, in the second phase of the simulation, the inflow profile of a heartbeat, cf. Figure 17, is applied periodically; see Section 4.3 .

\subsubsection{Software}

We use the LifeV software library 3.6.2 [42] coupled to the Finite Element Analysis Program (FEAP 8.2) [77] using a lightweight coupling library [40, 41], which is employed to synchronize and transfer data between the two software components.

\subsection{Initiating Physiological Blood Pressure (Ramp Phase)}

Before we start the simulation of heart beats, we apply an interior blood pressure of $80 \mathrm{mmHg}$ to the interior of the artery. This is crucial since the resulting prestretch has a strong influence on the fluidstructure interaction during the heart beat. We will initiate a slowly increasing blood flow (ramp), driven by imposing a flow rate at the inlet, until an internal pressure corresponding to roughly $80 \mathrm{mmHg}$ is reached. We refer to this part of the simulation as the ramp phase.

Since our goal is to reach a steady flow rate any oscillations are unwanted, may they be physical or only numerical artifacts. The shape and slope of the ramp affects the magnitude of oscillations. First, we therefore investigate the ramp phase of the FSI simulation with respect to different shapes and slopes of the ramp using different meshes and different finite element formulations. We expect to reach a steady state at a physiological blood pressure. This will be the prestressed physiological configuration of the coronary artery that will be used for the simulation of heart beats; see Section 4.3. We impose a flow rate at the inlet in the ramp phase featuring a parabolic inflow profile; see Figure 20. In the ramp phase, we choose a time step of $10^{-4} \mathrm{~s}$.

Already in this phase we observe differences between the different discretizations; see Section 4.2.2.

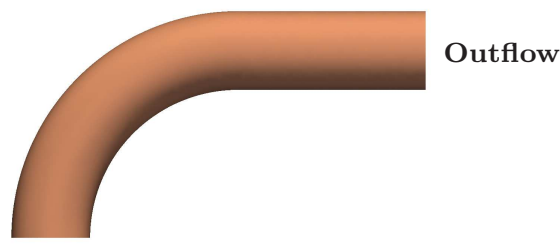

Inflow

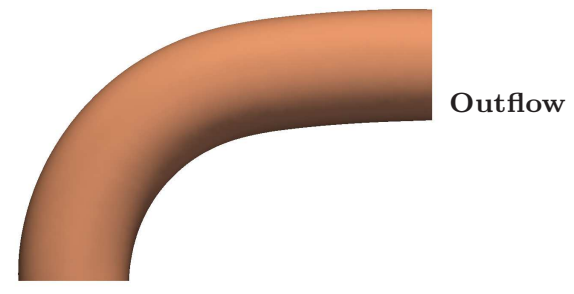

Inflow

Fig. 4 Geometry at a pressure of $0 \mathrm{mmHg}$ (left) and $80 \mathrm{mmHg}$ (right); displacement scaled by a factor of 3.0 . 

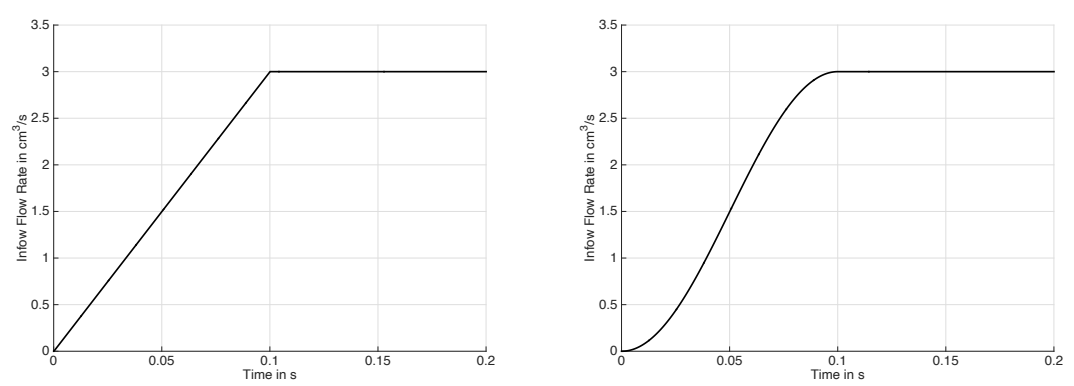

Fig. 5 Linear (left) and cosine (right) type ramp with $T_{\mathrm{R}}=0.1 \mathrm{~s}$.

\subsubsection{Linear Ramp}

We first choose a simple linear ramp, according to

$$
Q_{\text {ramp }}(t)= \begin{cases}\frac{t}{T_{\mathrm{R}}} Q_{\text {steady }} & \text { for } 0 \leq t \leq T_{\mathrm{R}} \\ Q_{\text {steady }} & \text { for } T_{\mathrm{R}} \leq t \leq T_{\text {steady }}\end{cases}
$$

cf. [8] and Figure 5 (left). In [8], a linear ramp with $T_{\mathrm{R}}=0.0177 \mathrm{~s}$ was already considered. Then, relatively high oscillations in all measured quantities, namely the flow rate, average pressure, and the cross sectional area have been observed. Additionally, the amplitude of the oscillations increased until the end of the simulation time for all depicted meshes. Thus, even though mesh convergence was observed, no steady state was reached in [8] within the simulation time.

Here, we consider seven different meshes, i.e., Mesh \#1 to Mesh \#7, cf. Table 4 and Table 5, with an increasing number of degrees of freedom. As we use unstructured meshes, we should note that the ratio of finite elements (and degrees of freedom) from one mesh to the next mesh is not constant. In this section, we restrict ourselves to $\mathrm{P} 1$ elements for comparison with the results in [8].

With respect to [8], $T_{\mathrm{R}}$ is increased and the inflow boundary condition was corrected. In [8], the change in the inflow cross sectional area during the simulation was not accounted for in the inflow profile, and this led to an incorrect flow rate profile over the cross sectional area. Additionally, the absorbing boundary condition has been adjusted, such that an outflow pressure of $80 \mathrm{mmHg}$ is reached at the outflow.

We measure the flow rate, the average pressure, and the cross sectional area at the inflow and the outflow, cf. Figure 2. The results of our simulations are presented in Figure 6. Compared to the results in [8], oscillations have been reduced considerably, and they now decrease during the simulation time. Besides, a pressure of approximately $80 \mathrm{mmHg}$, which is imposed by the absorbing boundary condition, is reached at the outflow, see Figure 6 (bottom, middle), while small but clearly visible oscillations are apparent.

The pressure at the inflow, see Figure 6 (top, middle), is slightly higher than the outflow pressure, cf. Figure 7. This is to be expected. In this figure, we also see that the frequency and amplitude of the oscillations in the pressure seem to be independent of the mesh size.

The inflow flow rate, which is imposed by a Dirichlet boundary condition, is now represented accurately in the simulation. For the outflow flow rate and pressure, see Figure 6 (bottom), small perturbations in the beginning of the ramp phase can be observed for the Meshes \#2, \#3, and \#4. At the first glance, they could be caused by the fact that the linear ramp is starting with a steep slope, and thus the first time steps are very difficult to solve. As we will discuss in Section 4.2.2, they indicate instabilities due to the $\mathrm{P} 1$ discretization, and are not caused by the shape of the ramp. 

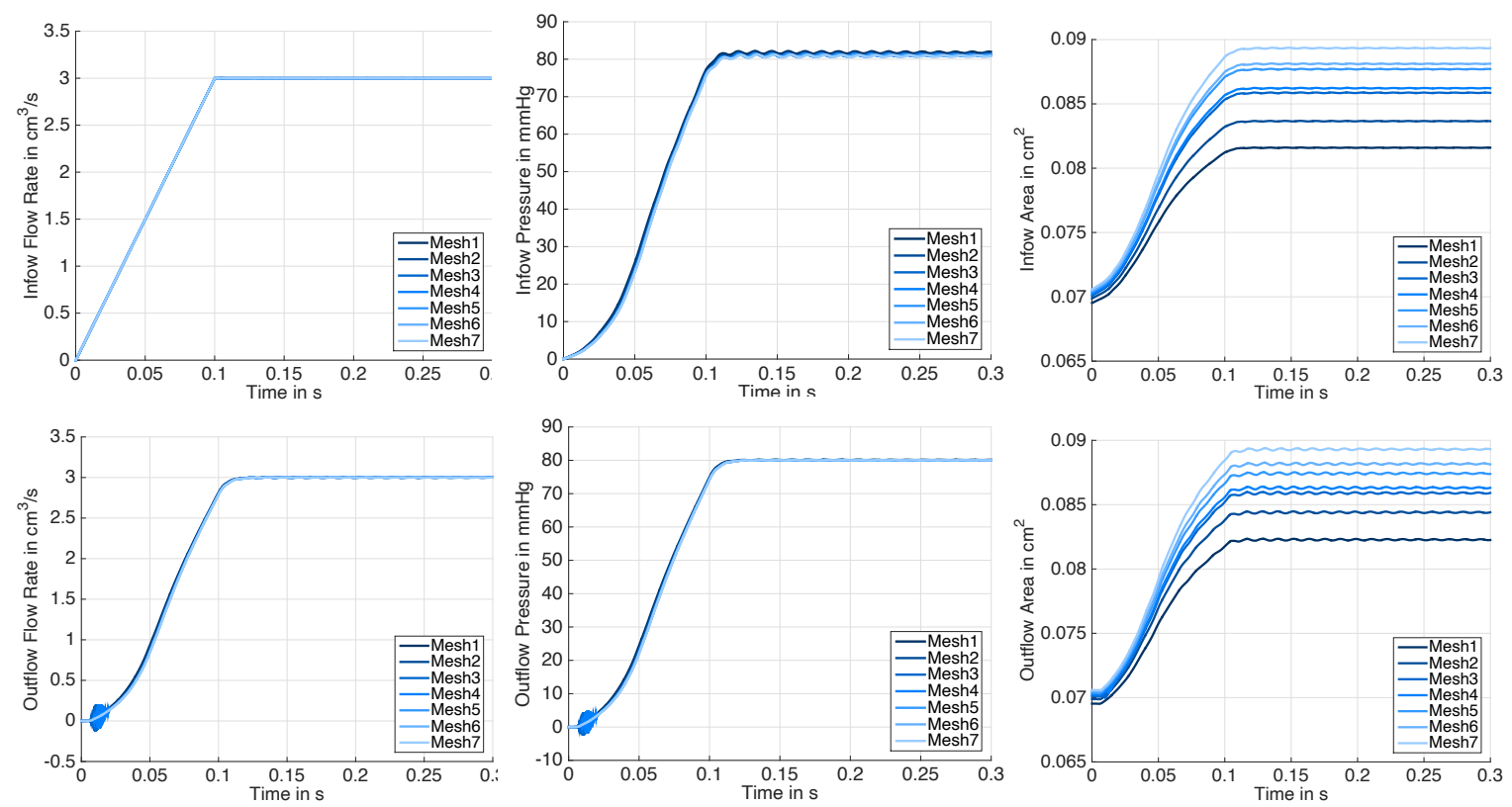

Fig. $6 \mathrm{P} 1$ mesh convergence study for the hyperelastic material using the linear ramp with $T_{\mathrm{R}}=0.1 \mathrm{~s}$. Flow rate (left), average pressure (middle), lumen cross sectional area (right), over time at the inlet (top) and outlet (bottom).

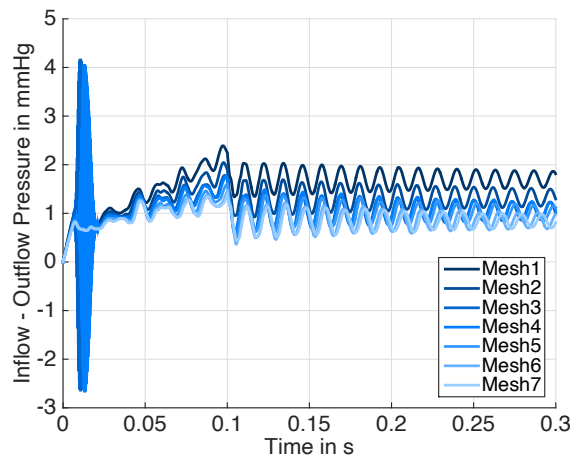

Fig. 7 Inflow minus outflow pressure for the hyperelastic material using the linear ramp with $T_{\mathrm{R}}=0.1 \mathrm{~s}$.

Similarly to the results in [8] the area is increasing when refining the mesh, but with much smaller oscillations. The cross sectional area, at the inflow as well as at the outflow, do not seem to converge when refining the meshes.

Within the simulation time of $0.3 \mathrm{~s}$, which corresponds to 3000 time steps, the oscillations decrease, but do not vanish. Because we want to start the heartbeat from a steady state configuration, using the linear ramp the simulation would have to be continued until the oscillations decrease further.

\subsubsection{Cosine-Type Ramp}

An alternative, better suited ramp, is 

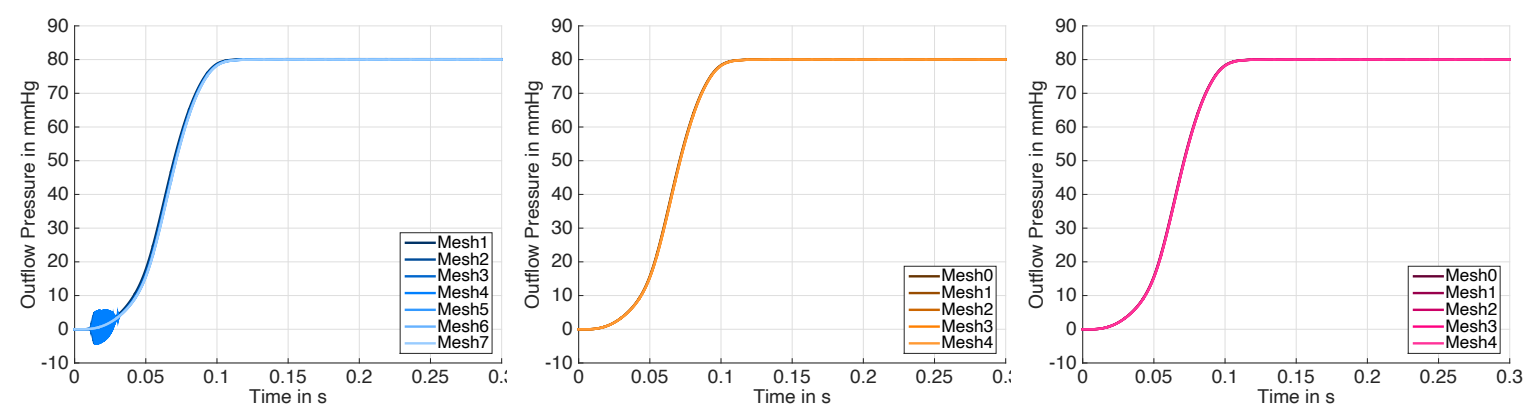

Fig. 8 Outflow pressure for the hyperelastic material using the cosine-type ramp with $T_{\mathrm{R}}=0.1 \mathrm{~s}$ for P1 (left), P2 (middle) and $\overline{\mathrm{F}}$ elements.
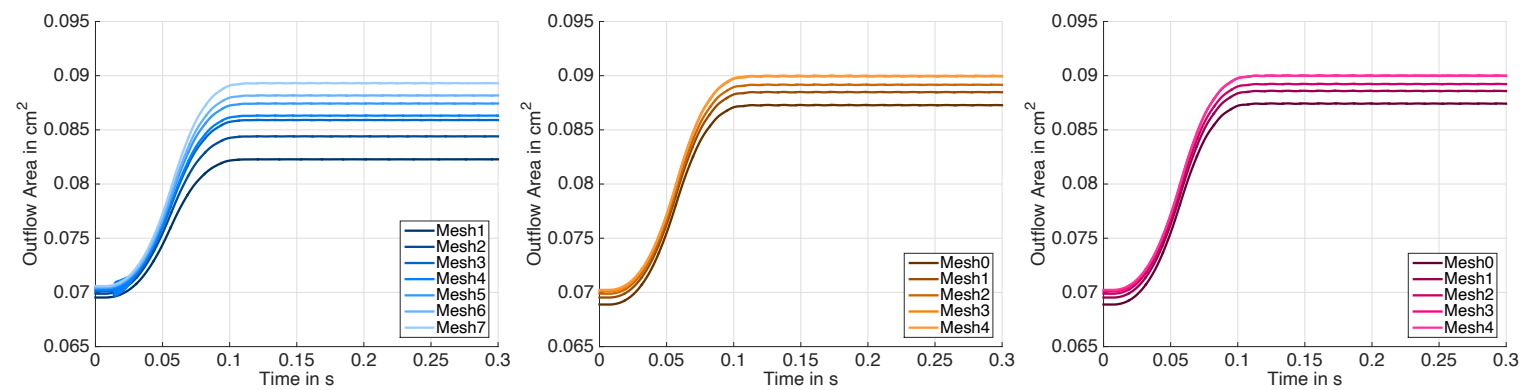

Fig. 9 Mesh convergence study of the outflow cross sectional area for the hyperelastic material using the cosine-type ramp with $T_{\mathrm{R}}=0.1 \mathrm{~s}$ for P1 (left), P2 (middle) and $\overline{\mathrm{F}}$ elements. In this diagram, the graphs for Mesh \#3 and Mesh \#4 completely overlap for $\mathrm{P} 2$ and $\overline{\mathrm{F}}$ elements.

$$
Q_{\text {ramp }}(t)= \begin{cases}\frac{1}{2} Q_{\text {steady }}\left(1-\cos \left(\frac{\pi}{T_{\mathrm{R}}} t\right)\right) & \text { for } 0 \leq t \leq T_{\mathrm{R}}, \\ Q_{\text {steady }} & \text { for } T_{\mathrm{R}} \leq t \leq T_{\text {steady }},\end{cases}
$$

cf. Figure 5. It is a $\mathcal{C}^{1}$-function which satisfies $\dot{Q}_{\text {ramp }}(0)=0$, meaning that the transition between the increasing and the constant part of the ramps is smooth, and that the difficulty of solving the first time steps is decreased.

Let us first discuss the perturbations in the outflow quantities. Therefore, we consider again for P1 elements the Meshes \#1 to \#7, and for P2 and $\overline{\mathrm{F}}$ elements the five meshes, i.e., Mesh \#0 to Mesh \#4, cf. Table 4 and Table 6. In Figure 8, the corresponding outflow pressure is displayed, and we can see that the perturbations remain also for this improved type of ramp. However, for P2 (middle) and $\overline{\mathrm{F}}$ (right) elements, the perturbations in the beginning of the ramp vanish. This shows clearly an improvement due to the use of these discretizations.

\begin{tabular}{|c|cccccccc|}
\hline Mesh & $\# 0$ & $\# 1$ & $\# 2$ & $\# 3$ & $\# 4$ & $\# 5$ & $\# 6$ & $\# 7$ \\
\hline Linear ramp P1 & - & $1.1 \cdot 10^{-4}$ & $1.2 \cdot 10^{-4}$ & $1.3 \cdot 10^{-4}$ & $1.3 \cdot 10^{-4}$ & $1.4 \cdot 10^{-4}$ & $1.2 \cdot 10^{-4}$ & $1.2 \cdot 10^{-4}$ \\
Cosine ramp P1 & - & $3.6 \cdot 10^{-6}$ & $8.6 \cdot 10^{-6}$ & $1.1 \cdot 10^{-5}$ & $1.3 \cdot 10^{-5}$ & $1.6 \cdot 10^{-5}$ & $1.9 \cdot 10^{-5}$ & $2.0 \cdot 10^{-5}$ \\
Cosine ramp P2 & $2.7 \cdot 10^{-5}$ & $2.8 \cdot 10^{-5}$ & $2.7 \cdot 10^{-5}$ & $2.7 \cdot 10^{-5}$ & $2.7 \cdot 10^{-5}$ & - & - & - \\
Cosine ramp F & $2.7 \cdot 10^{-5}$ & $2.8 \cdot 10^{-5}$ & $2.7 \cdot 10^{-5}$ & $2.7 \cdot 10^{-5}$ & $2.7 \cdot 10^{-5}$ & - & - & - \\
\hline
\end{tabular}

Table 7 Amplitude of the oscillations of the outflow cross sectional area in $\mathrm{cm}^{2}$ at $t=0.2 \mathrm{~s}$.

Another purpose for the use of the cosine-type ramp is to reduce oscillations in the constant part of the ramp phase. In Figure 9, the outflow cross sectional area for P1, P2, and $\overline{\mathrm{F}}$ elements is displayed for different meshes. Comparing these results to those of Figure 6, on the visible scale we appreciate a 

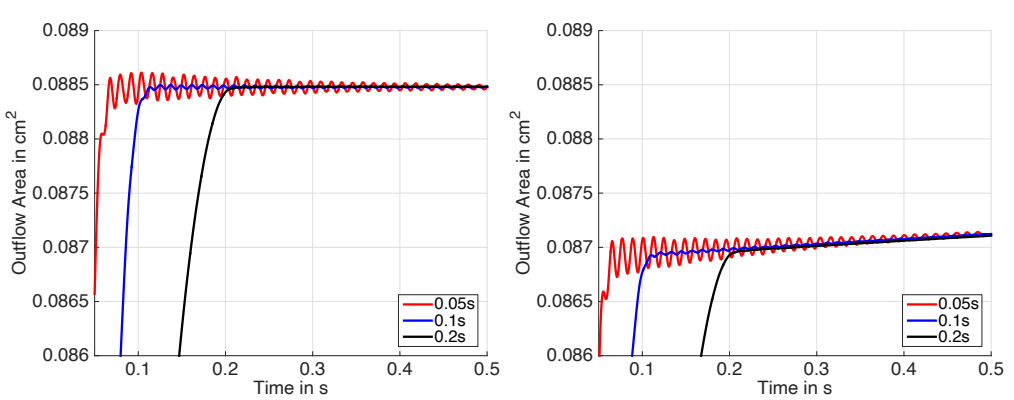

Fig. 10 Outflow cross sectional area using P2 elements on Mesh \#1. Hyperelastic (left), and viscoelastic (right) material model using parameter Set 2 from Table 3.

substantial reduction of the amplitude of the oscillations. More precisely, the amplitude is reduced by one order of magnitude, cf. Table 7, using the cosine-type ramp instead of the linear one.

In order to investigate mesh convergence, we again consider Figure 9. As in Figure 6, mesh convergence can not yet be observed for P1 elements (left). On the other hand, the results for P2 (middle) and $\overline{\mathrm{F}}$ (right) finite elements do suggest mesh convergence. Moreover, P2 and $\overline{\mathrm{F}}$ finite elements show a very similar behavior in Figure 8 and Figure 9, and there is a difference to P1 elements as can be seen in the latter figure. We will further discuss this in Section 4.2.4.

From the results presented here, from now on, we proceed using a cosine-type ramp to reduce oscillations. Next, we investigate whether we can further decrease $T_{\mathrm{R}}$.

\subsubsection{Steepness of the Ramp}

In this section, we will consider three different slopes of cosine-type ramps, i.e., $T_{\mathrm{R}} \in\{0.05 \mathrm{~s}, 0.1 \mathrm{~s}, 0.2 \mathrm{~s}\}$, in order to study the sensitivity. We again consider the oscillations of the outflow cross sectional area, and restrict ourselves to Mesh \#1 for P2 elements. In addition to the hyperelastic material model, here we also consider the viscoelastic material model, cf. Section 2. The complete set of results regarding the steepness of the ramp can be found in Appendix 6, including results for P1 elements.

We remark that the relaxation time of the viscoelastic material model is longer than $2 \mathrm{~s}$, and thus it cannot be at steady state after $0.5 \mathrm{~s}$. For the parameter Set 1 of Table 3, which has been used for the simulation, the overstresses are small and the relaxation time is long. Thus, viscoelastic effects are difficult to observe.

In Figure 10, oscillations are only visible for the shortest ramp. Until the end of the simulation time, i.e., 0.5s, these oscillations are still visible. For both longer ramps the oscillations are not visible at the scale presented, and thus considered as acceptable. The qualitative behavior of the hyperelastic and the viscoelastic material is similar, however, we can observe that the cross sectional area is lower for the viscoelastic material and slowly increases due to creep behavior. We will not focus on the differences of the material models now, but refer to the next section, which will discuss the influence of viscoelasticity in the fluid-structure interaction simulations with regard to the chosen space discretization.

As a result of the discussions so far, we will subsequently use a cosine-type ramp with a length of $T_{\mathrm{R}}=0.1 \mathrm{~s}$. After another $0.2 \mathrm{~s}$ of constant inflow flow rate we consider the system to be at steady state, i.e., $T_{\text {steady }}=0.3 \mathrm{~s}$, and ready for the subsequent simulation of a heartbeat. All further presented simulations will be performed using this ramp. 

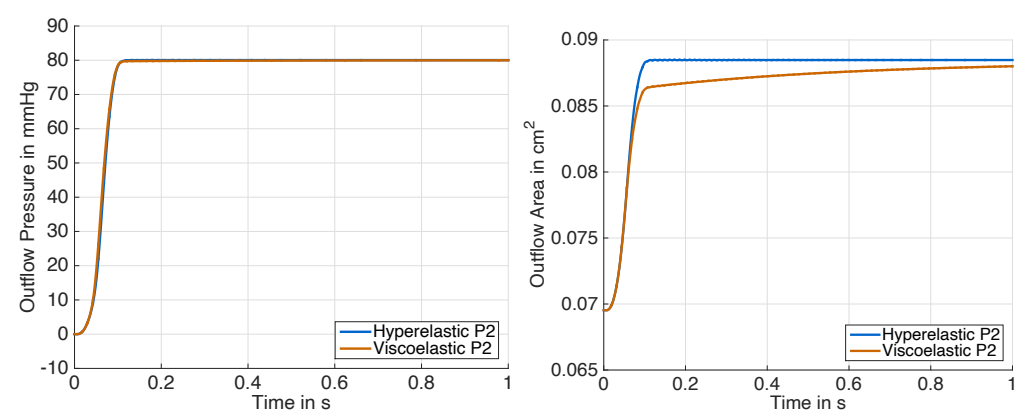

Fig. 11 Comparison of the hyperelastic and the viscoelastic for P2 elements material using the cosine-type ramp for parameter Set 2 from Table 3 on Mesh \#1. Outflow average pressure (left), outflow lumen cross sectional area (right).
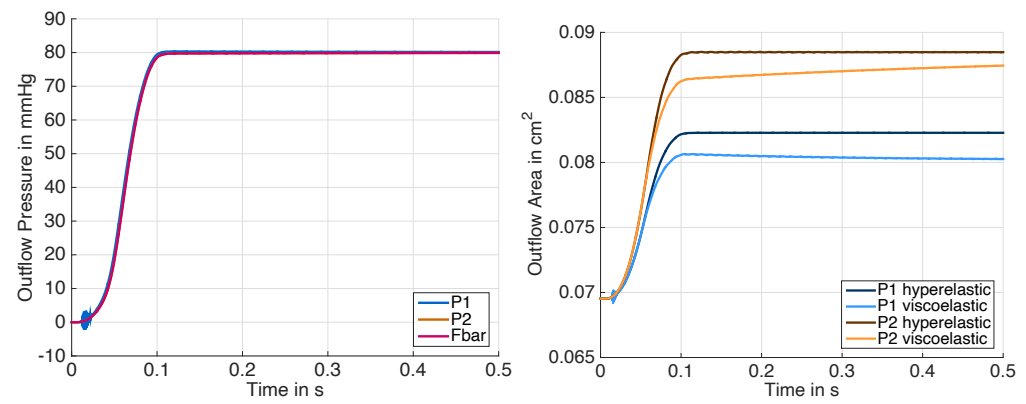

Fig. 12 Viscoelastic material on Mesh \#1, outflow pressure (left). Note that the curves for P2 and $\overline{\mathrm{F}}$ are almost identical. Comparison of the hyperelastic and the viscoelastic material model using the parameter Set 2 from Table 3 and P1 and P2 elements (right).

\subsubsection{Space Discretization and Viscoelastic Effects}

As already mentioned in Section 4.2.2, our results suggest that P2 and $\overline{\mathrm{F}}$ discretizations should be favored over simple P1 finite elements.

In this section, we compare the different space discretizations in detail. Using the viscoelastic material model we will see that P1 elements do not only show disadvantageous approximation properties but also show a qualitatively incorrect behavior.

Thus, first we present in Figure 11 the results of a simulation using the viscoelastic material model compared to the hyperelastic material model. In order to more clearly detect the viscoelasticity we use parameter Set 2 from Table 3, which has a much shorter relaxation time compared to Set 1 of Table 3. In Figure 11, the expected behavior can be observed: when we impose the same pressure (left) as for the hyperelastic material model, the displacement is lower in the beginning and converges to the displacement of the hyperelastic material model over time, cf. Section 2 and Figure 1. The displacement is here represented by the cross sectional area (right). This is caused by the creep behavior introduced in fiber direction, as described in Section 2.

In contrast to the appropriate results obtained with a P2 discretization, the use of a P1 discretization leads to a qualitatively wrong behavior, as can be seen in Figure 12: for a constant pressure (left), the displacement (right) decreases using P1 elements, while $\overline{\mathrm{F}}$ elements again yield the same correct results as P2 elements. Moreover, we can see that P2 elements yield the expected asymptotic behavior of the viscoelastic material model, while P1 elements do not.

We also observe that P1 elements lead to a much smaller displacement than P2 or $\overline{\mathrm{F}}$ elements for the same mesh. This can be seen for the hyperelastic and also the viscoelastic material model; cf. Figure 12 

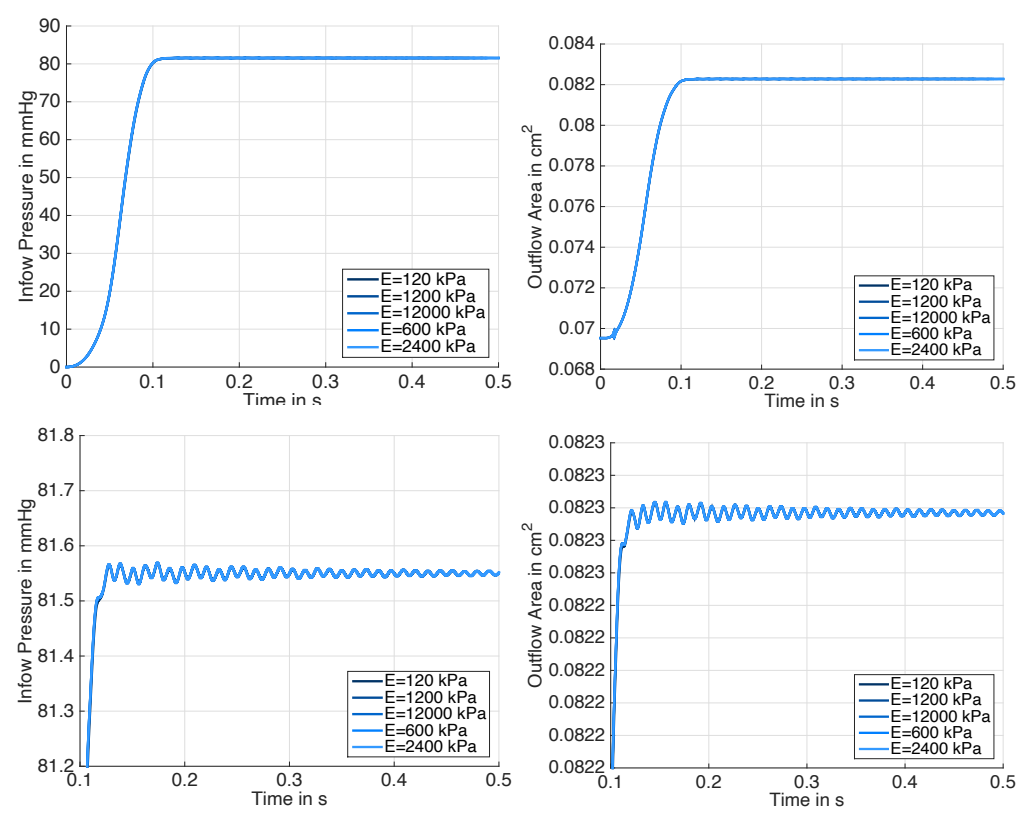

Fig. 13 Sensitivity analysis for the absorbing boundary conditions, performed with P1 elements using Mesh \#1: inflow average pressure (left), and outflow lumen cross sectional area (right). Global view (top), and zoom (bottom).

(right). We understand this behavior as the property of P1 elements to tend to locking effects and to be mechanically stiffer compared to P2 and $\overline{\mathrm{F}}$ elements.

These observations, in combination with the discussion in Section 4.2.2 about the perturbations which arise in the beginning of the ramp phase for P1 elements, are convincing arguments that P1 elements are not sufficient to describe the structural behavior accurately. Still it may be sufficient to consider P1 elements for the structure, if a hyperelastic material and very special quantities are to be analyzed, provided that a very fine mesh is considered.

\subsubsection{Sensitivity to Parameters of the Absorbing Boundary Condition}

A reason for the oscillations observed in Section 4.2.1, Section 4.2.2, and Section 4.2.3 could be the absorbing boundary condition, see Section 4.1.5, which we apply at the outflow to remove wave reflections. This absorbing boundary condition is based on a one dimensional linear elastic model. Since here we use highly nonlinear material models, it is not clear if the absorbing boundary condition will be able to completely remove wave reflections at the outlet, especially if the corresponding linear elastic material parameters are not chosen appropriately.

We investigate the influence of Young's modulus $E$ in the absorbing boundary condition. In order to minimize the computational effort, we used P1 elements and Mesh \#1 for these simulations. As can be seen in Figure 13, neither the inflow pressure (left) nor the outflow cross sectional area (right) are influenced strongly by the varying Young's modulus. These measured quantities showed the strongest oscillations in the previous sections, which are however relatively small due to the particular choice of the ramp. This suggests that the remaining oscillations in the constant part of the ramp are not caused by the absorbing boundary condition. 


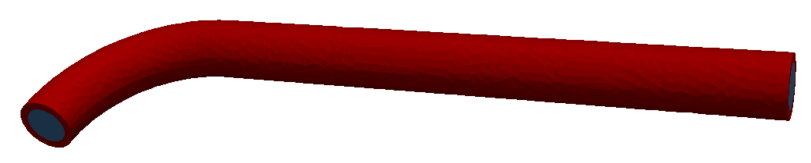

Fig. 14 Curved tube with a longer straight part $(4 \mathrm{~cm})$.

\subsubsection{Further Investigations on the Oscillations}

Our structure is statically determined only from applying Dirichlet boundary conditions at both ends. The surrounding tissue is thus neglected. The inflow of fluid into the curved geometry may therefore excite a bending mode of the structure.

We consider a new geometry, with a longer straight part of length $4 \mathrm{~cm}$, cf. Figure 14. The details of the mesh can be found in Tables 8 and 9 . We use a cosine ramp of length $T_{\mathrm{R}}=0.05 \mathrm{~s}$. As can be seen in Figure 15, there is indeed a strong bending of the tube over time.

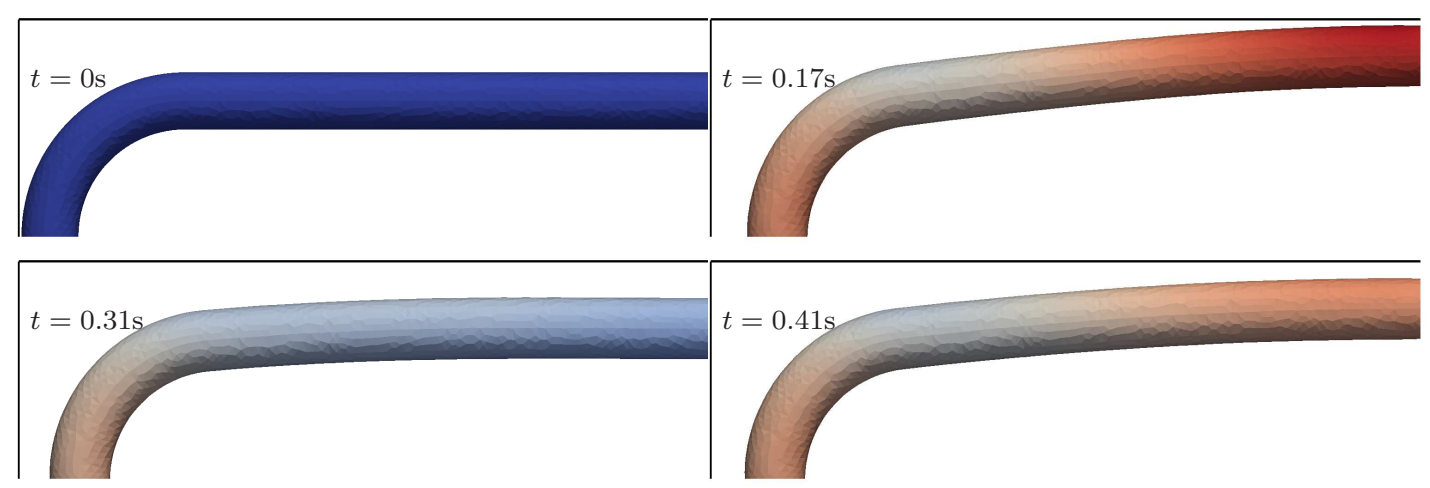

Fig. 15 Bending of the long tube.
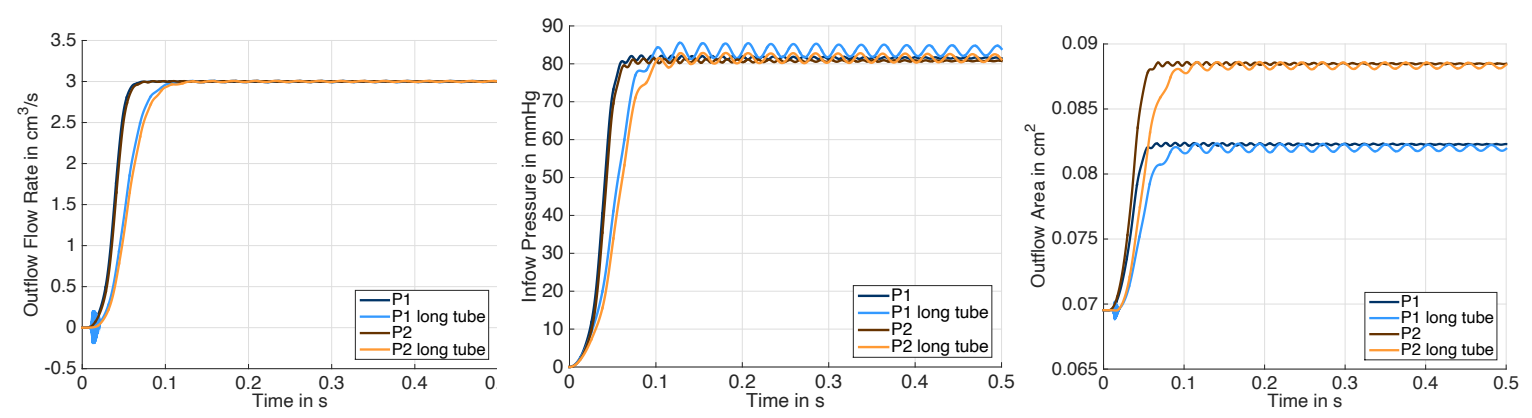

Fig. 16 Comparison of Mesh \#1 and the corresponding mesh of the long tube, cf. Tables 8 and 9 and Figure 14. Outflow flow rate (left), inflow average pressure (middle), outflow lumen cross sectional area (right).

In Figure 16, the outflow flow rate (left), inflow average pressure (middle), and outflow lumen cross sectional area (right) are shown for P1 and P2 elements, comparing the geometry described in Figure 2 and the similar geometry with a longer straight section. As expected, the outflow flow rate of the long tube has a delay compared to the standard geometry, because it takes longer for the fluid wave to reach the outflow of the tube. 


\begin{tabular}{|r|r|r|r|r|}
\hline $\begin{array}{r}\text { \#Fluid } \\
\text { elements }\end{array}$ & $\begin{array}{r}\text { \#Structural } \\
\text { elements }\end{array}$ & $\begin{array}{r}\text { Total } \\
\text { Dofs "P1" }\end{array}$ & $\begin{array}{r}\text { Total } \\
\text { Dofs "P2" }\end{array}$ & $\begin{array}{r}\text { Total } \\
\text { Dofs "F" } "\end{array}$ \\
\hline 2404 & 12348 & 64999 & 391693 & 391693 \\
\hline
\end{tabular}

Table 8 Degrees of freedom of the meshes corresponding to the long tube; see Figure 14.

\begin{tabular}{|r|r|r|r|r|r|}
\hline Discretization & Dofs $\mathbf{u}$ & Dofs $p$ & Dofs $\mathbf{d}_{s}$ & Dofs $\boldsymbol{\lambda}$ & Dofs $\mathbf{d}_{f}$ \\
\hline $\mathrm{P} 1$ & 10641 & 3547 & 32232 & 7938 & 10641 \\
$\mathrm{P} 2 / \overline{\mathrm{F}}$ & 65745 & 3547 & 224784 & 31872 & 65745 \\
\hline
\end{tabular}

Table 9 Degrees of freedom of the meshes corresponding to the long tube: fluid velocity $(\mathbf{u})$, fluid pressure $(p)$, structural displacement $\left(\mathbf{d}_{s}\right)$, coupling $(\boldsymbol{\lambda})$, and geometry/fluid mesh motion $\left(\mathbf{d}_{f}\right)$; see Figure 14 .

The oscillations arising in the inflow pressure and the outflow cross sectional lumen area show a much larger amplitude and also a frequency which is roughly smaller by a factor of $1 / 4$. Thus, the oscillations may depend on the length of the geometry. The fact that the amplitude of the oscillations increases significantly could also be an indication that the source of oscillations is a bending mode.

Now that we have discussed the ramp, we believe that a reasonable way to carry out the simulation of the ramp phase, including the type and length of the ramp, and a reasonable space discretization have been found. In the next sections, we are going to concentrate on the simulation of heartbeat(s) after the ramp phase.

\subsection{Simulation of Several Heartbeats}

We now present and discuss our results for several full heartbeats. In advance of the heartbeat phase, the artery has been prestretched up to a physiological pressure of $80 \mathrm{mmHg}$. Referring to the Sections 4.2 .2 and 4.2.3, we choose a cosine-type ramp with length $T_{\mathrm{R}}=0.1 \mathrm{~s}$ in order to inflate the tube. As already mentioned in previous sections, we continue the simulation at a constant inflow flow rate $Q_{\text {steady }}$ until $0.3 \mathrm{~s}$, and consider the system to be at steady state at this point of simulation time. We neglect some very small oscillations still remaining after $0.3 \mathrm{~s}$; also cf. Figure 10 for $T_{\mathrm{R}}=0.1 \mathrm{~s}$.

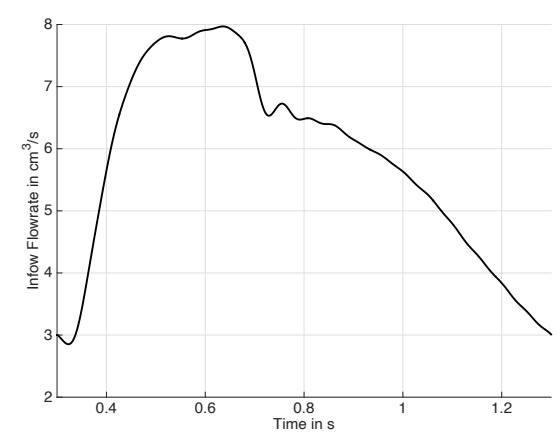

Fig. 17 Inflow flow rate for the heartbeat phase.

Several heartbeats follow, i.e., we impose a flow rate profile $Q_{\text {heartbeat }}$ over time according to Figure 17. This inflow profile was created using an inflow pressure profile taken from [1]. It corresponds to a single heartbeat and the duration is $1.0 \mathrm{~s}$. We use the inflow pressure function as a non-homogeneous Neumann 

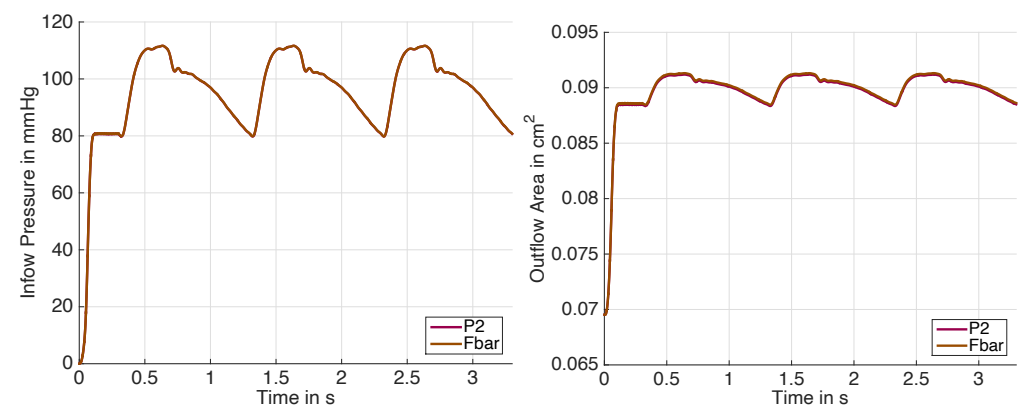

Fig. 18 Simulation of 3 heartbeats using Mesh \#1 and the hyperelastic material model: Inflow pressure (left), and outflow cross sectional lumen area (right).

structural displacement in $\mathrm{cm}$

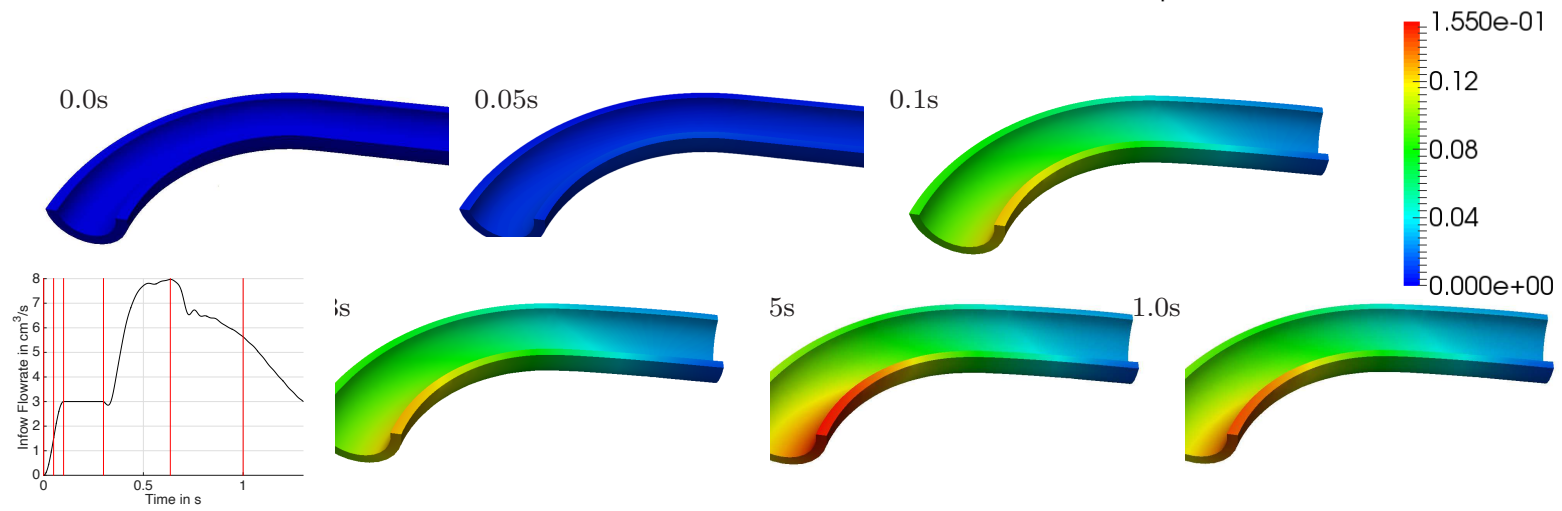

Fig. 19 Evolution of the displacement magnitude of the structure for Mesh \#3 and $\overline{\mathrm{F}}$ elements in the deformed configuration, at times $0.0 \mathrm{~s}$ (top left), 0.05s (top middle), 0.1s (top right), 0.3s (bottom left), 0.635s (bottom middle), and 1.0s (bottom right). Displacement is scaled by a factor of 2.0 .

boundary condition at the inflow for a single simulation done with P1 elements on Mesh \#1. The inflow flow rate over time resulting from this simulation was then approximated by means of a Fourier series of order 20, and thus a periodic function $Q_{\text {heartbeat }}$ was obtained. This function describes the inflow flow rate over time during each heartbeat, cf. Figure 17. Analogously to the inflow flow rate profile $Q_{\text {ramp }}$, used for the ramp phase, we use $Q_{\text {heartbeat }}$ as a Dirichlet boundary condition in the second part of the simulation.

During the heartbeat, we choose a time step much larger than in the ramp phase, i.e., $\Delta t=10^{-3} \mathrm{~s}$. In Figure 18, the average inflow pressure (left) and outflow cross sectional lumen area (right) for P2 and $\overline{\mathrm{F}}$ elements are presented. Similarly to the ramp phase, cf. Section 4.2.4, also during all three heartbeats, we see a very similar behavior for both discretizations.

In Figure 19 and 20, the deformation and flow rate profile over time is depicted. The largest deformation is observed at the inner part of the curvature, near the inlet. Here, the tube deforms most due to the increasing flow rate at the inlet. Considering the flow rate, we can see that the flow is faster at the outer part of the curved pipe.

\subsection{Mesh Convergence for the Heartbeat}

In this section, we address the influence of the mesh refinement during the heartbeat phase, analogously to the discussions in Section 4.2.2 and Section 4.2.1 about the ramp phase. 

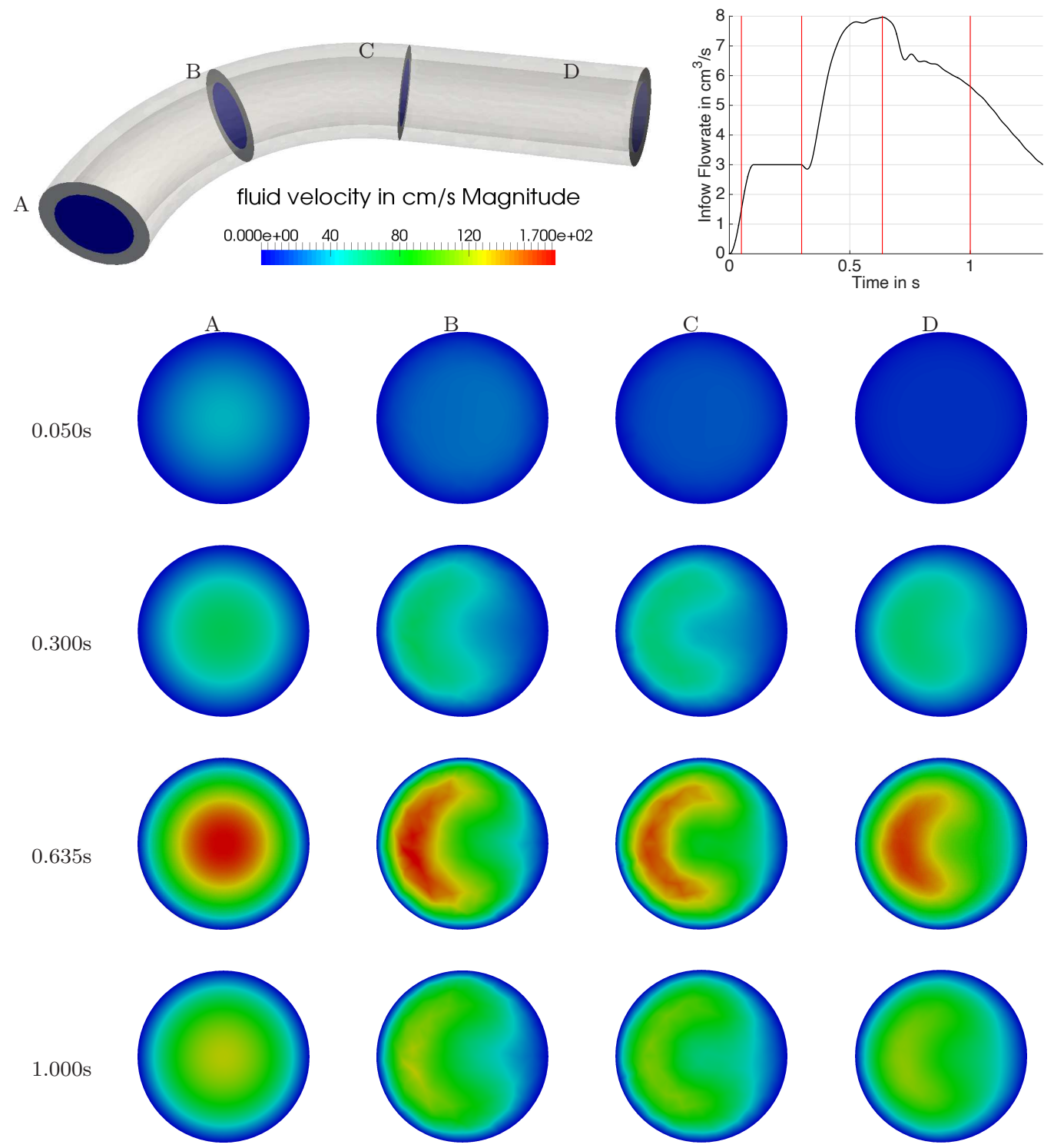

Fig. 20 Evolution of the flow for Mesh \#7 and P1 elements at different slices.

In Figure 21, mesh convergence plots for P1 (left), P2 (middle), and $\overline{\mathrm{F}}$ (right) elements are shown. We show Meshes \#1 to \#7 for P1 elements and Mesh \#0 to \#4 for P2 and $\overline{\mathrm{F}}$ elements. Mesh \#4 is plotted only until $0.6 \mathrm{~s}$.

Considering the outflow area, for P1 elements, we do not see mesh convergence. For P2 and $\overline{\mathrm{F}}$ finite elements, the graphs for Mesh \#3 and Mesh \#4 overlap. Considering the inflow pressure plots (top), for all element types, there are small differences in the pressure for the different meshes because we do not impose a pressure at steady state here anymore. The differences are clearly visible in the systolic phase of the heartbeat, whereas the inflow pressure is very close to each other for all meshes during the diastolic part. Thus, especially the maximal pressure decreases when refining the mesh. For P1, the pressure of the coarsest mesh is significantly higher than the maximal pressure of the coarsest P2 or $\overline{\mathrm{F}}$ mesh. We conclude that for P1 elements, we are still far away from asymptotics, while for P2 and $\overline{\mathrm{F}}$ elements, we 

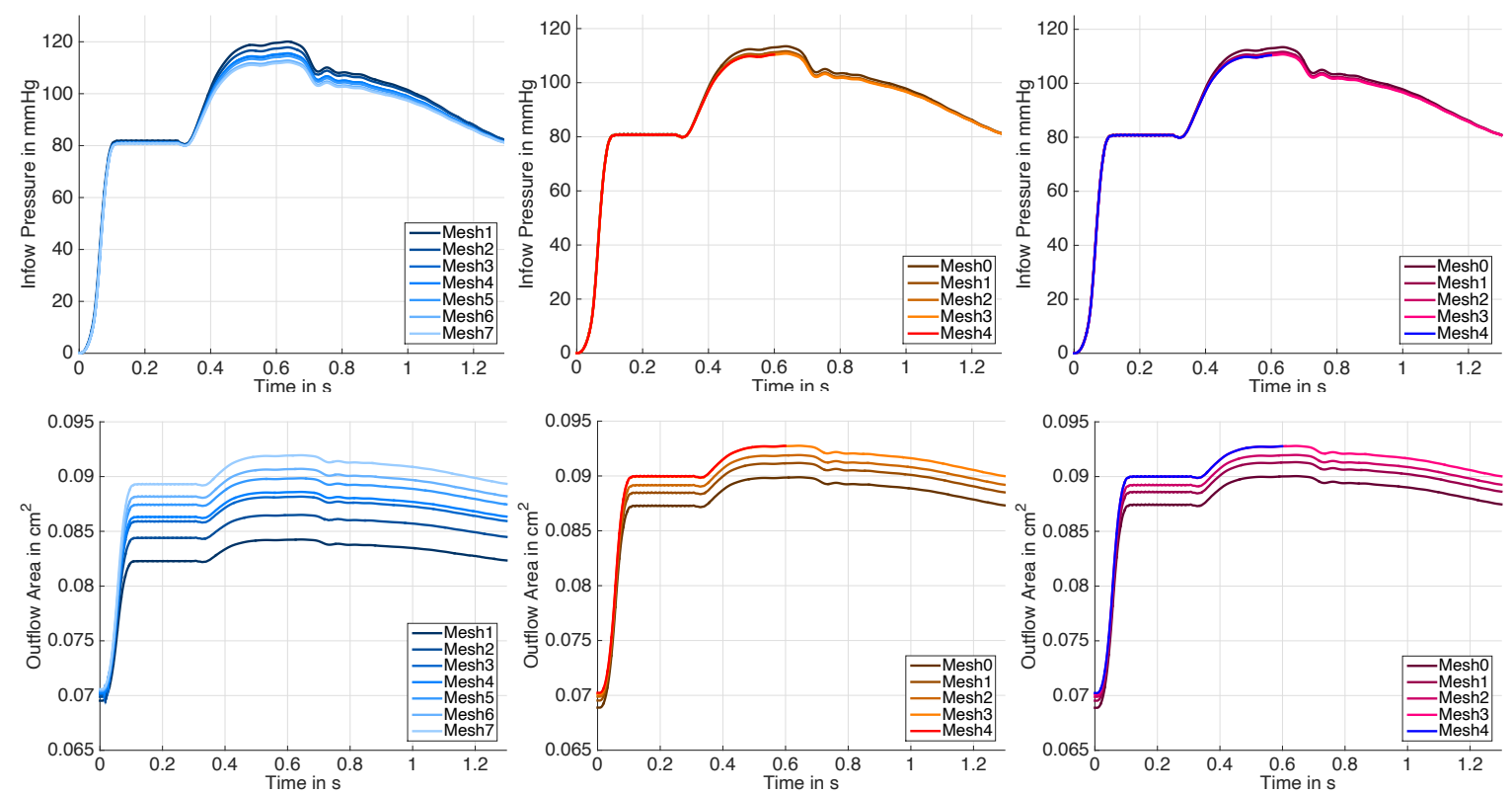

Fig. 21 Mesh convergence of the inflow pressure (top) and outflow cross sectional lumen area (bottom) during the heartbeat phase: P1 (left), P2 (middle) and $\overline{\mathrm{F}}$ (right) elements.

have indication of mesh convergence: The graphs for Mesh \#3 and Mesh \#4 completely overlap for all quantities.
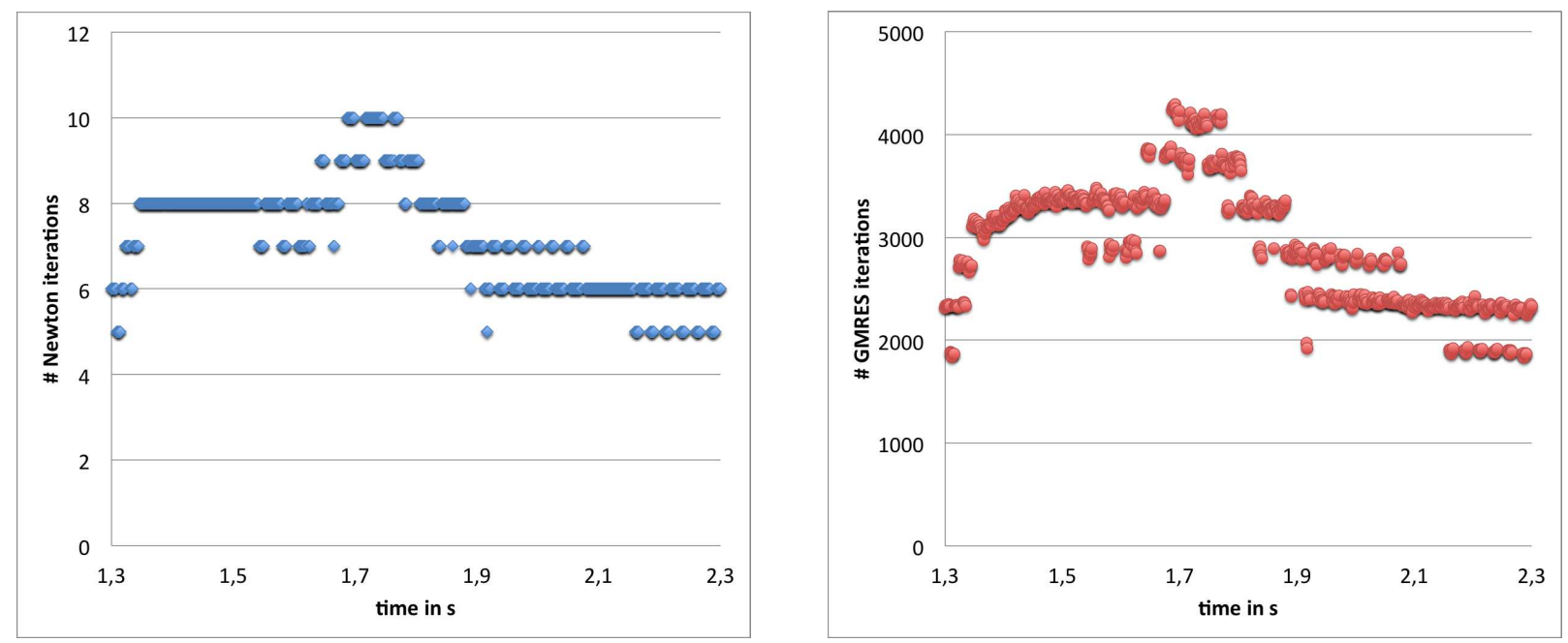

Fig. 22 Number of Newton iterations for each time step (left) and sum of the GMRES iterations in each time step (right) during the heart beat using Mesh \#3 and $\overline{\mathrm{F}}$ finite elements. We use 96 processors and thus 96 subdomains for the overlapping Schwarz method within our monolithic Dirichlet-Neumann preconditioner.

In Figure 22, we present the number of Newton iterations for each time step as well as the sum of GMRES iterations in each time step, using Mesh \#3 and $\overline{\mathrm{F}}$ finite elements. Improvements of the monolithic preconditioner and of the overlapping Schwarz method, to reduce the number of GMRES iterations, are ongoing work; first results have been obtained. 

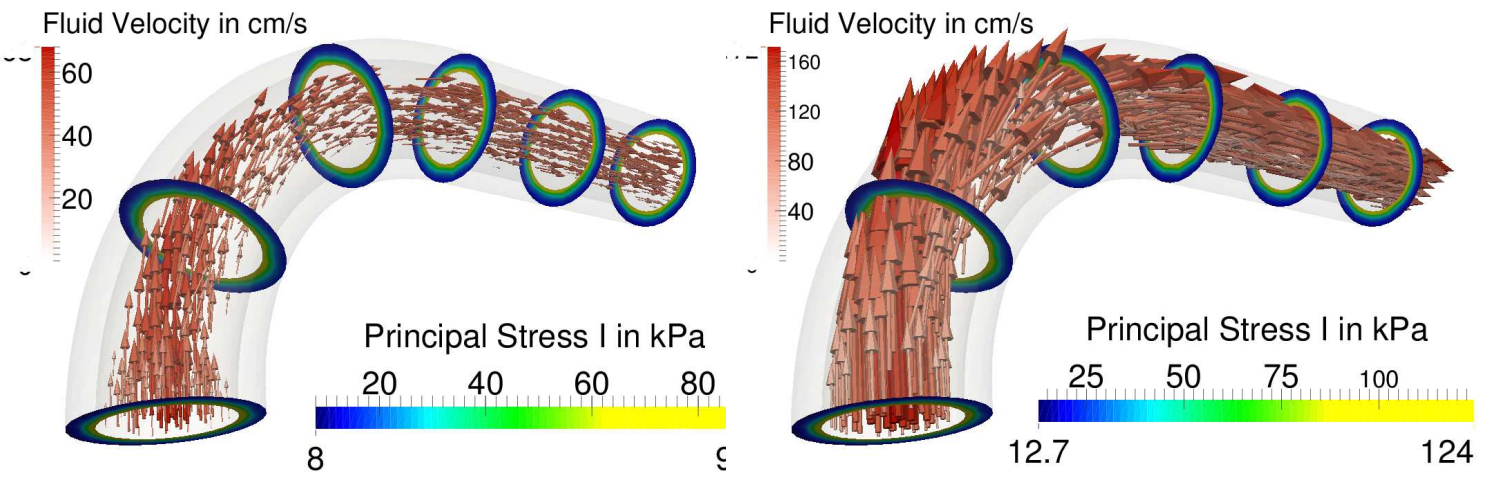

Fig. 23 Fluid velocity and first principal Cauchy stress at $t=0.3 \mathrm{~s}$ (left) and at $t=0.635 \mathrm{~s}$ (right); cf. Fig 20.

\subsection{Analysis of Stresses}

In this section, the transmural stress distribution inside the arterial wall is investigated. Therefore, a simulation, including a cosine ramp with $T_{\mathrm{R}}=0.1 \mathrm{~s}$ followed by a heartbeat starting at $t=0.3 \mathrm{~s}$, is considered. For the structural response our polyconvex, anisotropic hyperelastic material model is applied; cf. Section 2. In order to analyze the quality of the approximated stress quantities all different element formulations P1, P2 and $\overline{\mathrm{F}}$ are considered and compared to each other. In case of the P1 discretization Mesh \#7 is used, whereas for the P2 and $\overline{\mathrm{F}}$ simulations Mesh \#3 is taken into account here. Following the findings from Section 4.4 these meshes are considered to yield sufficiently accurate solutions in the sense of mesh convergence. In detail the stress quantities are evaluated at two different times during the simulation, i.e., $t=0.3 \mathrm{~s}$ and $t=0.635 \mathrm{~s}$. These correlate with the steady state right before the beginning of the heartbeat, representing a diastolic blood pressure of $80 \mathrm{mmHg}$, and with the peak value of the inflow flow rate for the heartbeat phase, where a systolic blood pressure of approximately $120 \mathrm{mmHg}$ is reached. An illustration of the evaluation points and the associated fluid velocity and transmural distribution of the first principal Cauchy stresses, at different slices of the geometry, is shown in Figure 23. Here the results from the simulation using the P2 elements are depicted. Even though the given geometry in the reference configuration is rotationally symmetric with respect to the axis orthogonal to the respective cross section, the evaluation of circumferential, axial and radial stresses in the current configuration may not be straightforward due to the deformation induced by the blood flow. Here, however, the first, second, and third principal normal stresses coincide almost perfectly with the normal stresses in circumferential, axial and radial direction, respectively. Figure 24 shows the first principal Cauchy stresses, where the geometry has been clipped in the $\mathrm{x}-\mathrm{z}$ plane such that the inner surface as well as the stress distribution over the wall thickness is displayed. The P1 discretization shows clearly higher stress values, which strongly oscillate on the inner surface of the tube. This observation highlights the stiffness of P1 elements and their sensitivity with respect to locking effects; also cf. the discussion on the $\bar{F}$-discretization and potential locking in Section 3.3. On the other hand the $\overline{\mathrm{F}}$ and $\mathrm{P} 2$ discretizations show a smoother stress distribution. This significant difference in the stresses and also the heavy checker-boarding of P1 emphasizes to not use P1 elements but at least quadratic shape functions.

The non-symmetric flow profiles visible in Figure 20 are however hardly reflected by the principal stress distributions. This is most probably due to a pressure-dominated response reflected by these stresses. Figure 25 presents the maximal shear stresses in the fluid-solid interface plane, which are obtained by rotating the local principle stress directions by $45^{\circ}$ around the radial axis. As can be seen, these shear stresses are higher at the inner curve and represent therefore a non-rotationally symmetric stress distribution. These observations correspond well with common hypotheses stating that the plaque evolves where low flow rates 
Numerical Modeline of Fluid-Structure Interaction in Arteries at Finite Strains

29
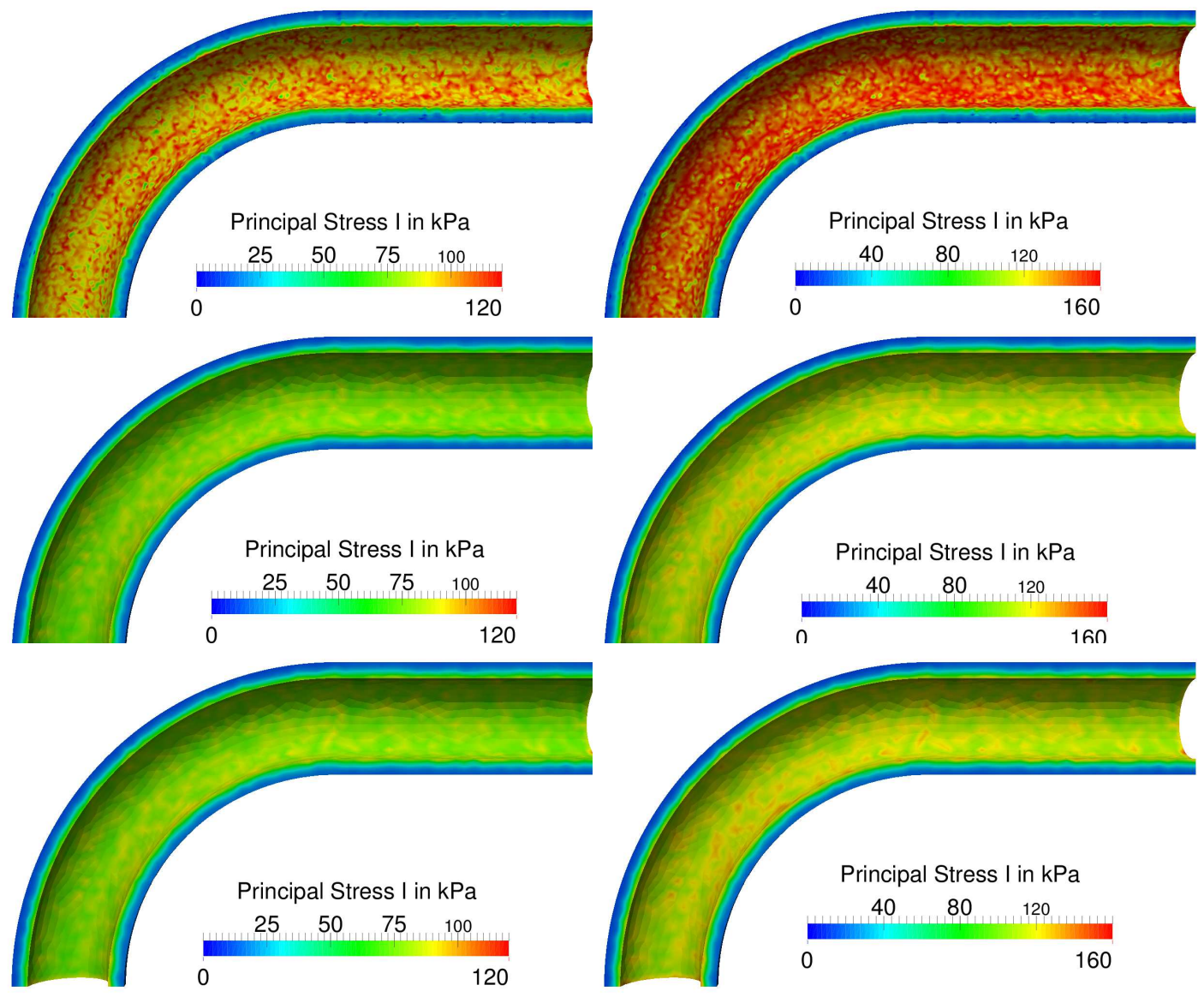

Fig. 24 Comparison of the first principal Cauchy stress for P1, P2 and $\overline{\mathrm{F}}$ : P1 at $t=0.3 \mathrm{~s}$ (top left), P1 at $t=0.635 \mathrm{~s}$ (top right), $\mathrm{P} 2$ at $t=0.3 \mathrm{~s}$ (middle left), $\mathrm{P} 2$ at $t=0.635 \mathrm{~s}$ (middle right), $\overline{\mathrm{F}}$ at $t=0.3 \mathrm{~s}$ (bottom left), $\overline{\mathrm{F}}$ at $t=0.635 \mathrm{~s}$ (bottom right).
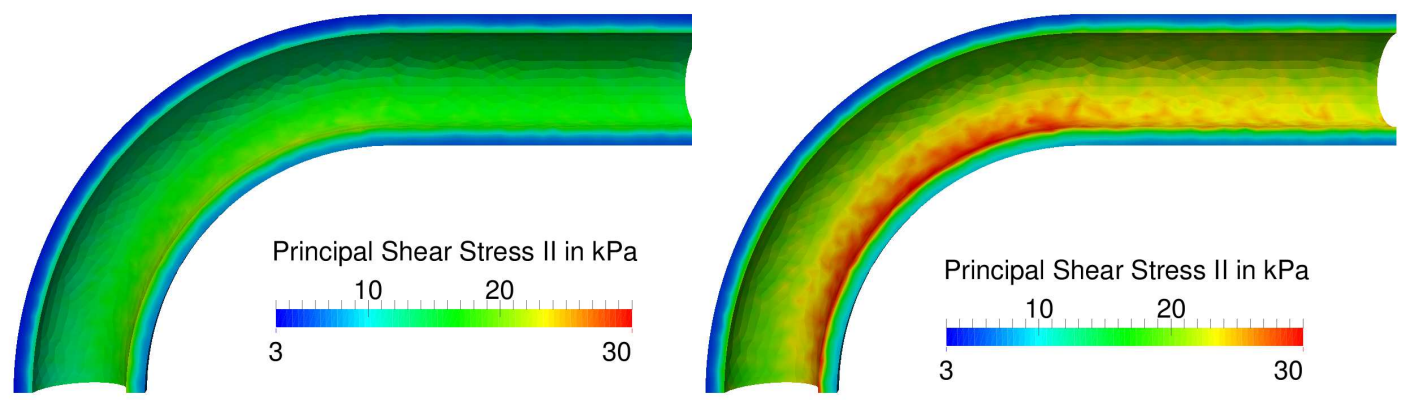

Fig. 25 The principal Cauchy shear stress using the $\overline{\mathrm{F}}$ element at $t=0.3 \mathrm{~s}$ (left) and $t=0.635 \mathrm{~s}$ (right).

and high shear stresses are found in domains close to the endothelial cells, which is mostly at the inner curves of vessel walls. 


\section{Acknowledgment}

Financial support by the German Science Foundation (DFG), project no. BA2823/9-1, KL2094/3-1, RH122/4-1, and SCHR570/15-1, and the Swiss National Foundation (SNF), project no. 140184 is gratefully appreciated. Furthermore, Daniel Balzani appreciates funding from the DFG in the context of the Institutional Strategy - The Synergetic University, at TU Dresden, as part of the Excellence Initiative. Alexander Heinlein, Axel Klawonn, and Oliver Rheinbach acknowledge the use of the CHEOPS parallel computer at Universität zu Köln, the Cray XT6m at the University of Duisburg-Essen, the Cluster at Technische Universität Bergakademie Freiberg and the Oculus cluster at Universität Paderborn. Simone Deparis, Davide Forti and Alfio Quarteroni acknowledge the Swiss National Supercomputing Center (CSCS) for providing CPU resources under project ID s475.

\section{References}

1. Hemolab, 2014. http://hemolab.lncc.br/adan-web.

2. S. Badia, A. Quaini, and A. Quarteroni. Splitting methods based on algebraic factorization for fluid-structure interaction. SIAM J. Sci. Comput., 30(4):1778-1805, 2008.

3. J. Ball. Convexity conditions and existence theorems in non-linear elasticity. Arch. Ration. Mech. Anal., 63:337-403, 1977.

4. D. Balzani. Polyconvex Anisotropic Energies and Modeling of Damage Applied to Arterial Walls. Phd thesis, University Duisburg-Essen, Verlag Glückauf Essen, 2006.

5. D. Balzani, D. Böse, D. Brands, R. Erbel, A. Klawonn, O. Rheinbach, and J. Schröder. Parallel simulation of patientspecific atherosclerotic arteries for the enhancement of intravascular ultrasound diagnostics. Engineering Computations, $29(8), 2012$.

6. D. Balzani, D. Brands, A. Klawonn, and O. Rheinbach. Large-scale simulation of arterial walls: mechanical modeling. PAMM, 7(1):4020017-4020018, 2007. Special Issue: Sixth International Congress on Industrial Applied Mathematics (ICIAM07) and GAMM Annual Meeting, Zürich 2007.

7. D. Balzani, D. Brands, A. Klawonn, O. Rheinbach, and J. Schröder. On the mechanical modeling of anisotropic biological soft tissue and iterative parallel solution strategies. Archive of Applied Mechanics, 80(5):479-488, 2010.

8. D. Balzani, S. Deparis, S. Fausten, D. Forti, A. Heinlein, A. Klawonn, A. Quarteroni, O. Rheinbach, and J. Schröder. Aspects of arterial wall simulations: Nonlinear anisotropic material models and fluid structure interaction. In Proceedings of the WCCM XI, 2014.

9. D. Balzani, F. Gruttmann, and J. Schröder. Analysis of thin shells using anisotropic polyconvex energy densities. Comput. Methods Appl. Mech. Engrg., 197:1015-1032, 2008.

10. D. Balzani, P. Neff, J. Schröder, and G. A. Holzapfel. A polyconvex framework for soft biological tissues. Adjustment to experimental data. Internat. J. Solids Structures, 43(20):6052-6070, 2006.

11. D. Balzani, J. Schröder, D. Brands, A. Klawonn, and O. Rheinbach. Computer simulation of damage in overstretched atherosclerotic arteries. Proceedings of ICCB 2007 - III. International Congress on Computational Bioengineering, 2007.

12. A. T. Barker. Parallel monolithic fluid-structure interaction algorithms with application to blood flow simulation. PhD thesis, University of Colorado, 2009.

13. A. T. Barker and X.-C. Cai. Scalable parallel methods for monolithic coupling in fluid-structure interaction with application to blood flow modeling. J. Comput. Phys., 229(3):642-659, 2010.

14. Y. Bazilevs, V. M. Calo, T. J. R. Hughes, and Y. Zhang. Isogeometric fluid-structure interaction: theory, algorithms, and computations. Comput. Mech., 43:3-37, 2008.

15. Y. Bazilevs, K. Takizawa, and T. Tezduyar. Computational Fluid-Structure Interaction. Methods and Applications. Wiley Series in Computational Mechanics. Wiley, 2013.

16. M. Benzi and M. A. Olshanskii. An augmented Lagrangian-based approach to the Oseen problem. SIAM J. Sci. Comput., 28(6):2095-2113 (electronic), 2006.

17. M. Benzi, M. A. Olshanskii, and Z. Wang. Modified augmented lagrangian preconditioners for the incompressible NavierStokes equations. Int. J. Numer. Meth. Fluids, 2010.

18. J. Boehler. Introduction to the invariant formulation of anisotropic constitutive equations. In J. Boehler, editor, $A p$ plications of tensor functions in solid mechanics, number 292 in Courses and Lectures of CISM, pages 13-30. Springer, 1987.

19. D. Böse, S. Brinkhues, R. Erbel, A. Klawonn, O. Rheinbach, and J. Schröder. A simultaneous augmented lagrange approach for the simulation of soft biological tissue. In R. Bank, M. Holst, O. Widlund, and J. Xu, editors, Domain 
Decomposition Methods in Science and Engineering XX, volume 91 of Lecture Notes in Computational Science and Engineering, pages 369-376. Springer Berlin Heidelberg, 2013.

20. D. Brands, A. Klawonn, O. Rheinbach, and J. Schröder. Modelling and convergence in arterial wall simulations using a parallel FETI solution strategy. Comput. Methods Biomech. Biomed. Engin., 11:569-583, 2008.

21. S. Brinkhues, A. Klawonn, O. Rheinbach, and J. Schröder. Augmented lagrange methods for quasi-incompressible materials-applications to soft biological tissue. Internat. J. Numer. Methods Biomed. Engrg, 29(3):332-350, 2013.

22. E. Burman and M. A. Fernández. Continuous interior penalty finite element method for the time-dependent navier-stokes equations: space discretization and convergence. Numerische Mathematik, 107(1):39-77, 2007.

23. S. Čanić, C. Hartley, D. Rosenstrauch, J. Tambača, G. Guidoboni, and A. Mikelić. Blood flow in compliant arteries: An effective viscoelastic reduced model, numerics, and experimental validation. Annals of Biomedical Engineering, 34(4):575-592, 2006.

24. P. Causin, J. F. Gerbeau, and F. Nobile. Added-mass effect in the design of partitioned algorithms for fluid-structure problems. Comput. Methods Appl. Mech. Engrg., 194(42-44):4506-4527, 2005.

25. G.-H. Cottet, E. Maitre, and T. Milcent. Eulerian formulation and level set models for incompressible fluid-structure interaction. M2AN Math. Model. Numer. Anal., 42:471-492, 2008.

26. P. Crosetto, S. Deparis, G. Fourestey, and A. Quarteroni. Parallel algorithms for fluid-structure interaction problems in haemodynamics. SIAM J. Sci. Comput., 33(4):1598-1622, 2011.

27. P. Crosetto, P. Reymond, S. Deparis, D. Kontaxakis, N. Stergiopulos, and A. Quarteroni. Fluid-structure interaction simulation of aortic blood flow. Computers \& Fluids, 43(1):46-57, 2011.

28. J. Degroote, K.-J. Bathe, and J. Vierendeels. Performance of a new partitioned procedure versus a monolithic procedure in fluidstructure interaction. Computers \& Structures, 87(1112):793 - 801, 2009. Fifth MIT Conference on Computational Fluid and Solid Mechanics.

29. J. Degroote and J. Vierendeels. Multi-level quasi-newton coupling algorithms for the partitioned simulation of fluidstructure interaction. Comput. Methods Appl. Mech. Engrg., 225228(0):14 - 27, 2012.

30. S. Deparis, M. Discacciati, G. Fourestey, and A. Quarteroni. Fluid-structure algorithms based on Steklov-Poincaré operators. Comput. Methods Appl. Mech. Engrg., 195:5797-5812, 2006.

31. S. Deparis, D. Forti, and A. Quarteroni. A rescaled localized radial basis function interpolation on non-cartesian and nonconforming grids. SIAM J. Sci. Comput., 36(6):A2745-A2762, 2014.

32. T. Dunne. Adaptive Finite Element Approximation of Fluid-Structure Interaction Based on Eulerian and Arbitrary Lagrangian-Eulerian Variational Formulations. PhD thesis, Ruprechts-Karls Universität Heidelberg, 2007.

33. H. Elman, V. E. Howle, J. Shadid, R. Shuttleworth, and R. Tuminaro. Block preconditioners based on approximate commutators. SIAM J. Sci. Comput., 27(5):1651-1668 (electronic), 2006.

34. H. Elman, V. E. Howle, J. Shadid, R. Shuttleworth, and R. Tuminaro. A taxonomy and comparison of parallel block multi-level preconditioners for the incompressible Navier-Stokes equations. J. Comput. Phys., 227(3):1790-1808, 2008.

35. H. Elman, V. E. Howle, J. Shadid, D. Silvester, and R. Tuminaro. Least squares preconditioners for stabilized discretizations of the Navier-Stokes equations. SIAM J. Sci. Comput., 30(1):290-311, 2007.

36. M. Á. Fernández, L. Formaggia, J. F. Gerbeau, and A. Quarteroni. The derivation of the equations for fluids. In Cardiovascular Mathematics, pages 77-121. Springer, 2009.

37. M. Á. Fernández, J.-F. Gerbeau, A. Gloria, and M. Vidrascu. Domain decomposition based Newton methods for fluidstructure interaction problems. In CANUM 2006-Congrès National d'Analyse Numérique, volume 22 of ESAIM Proc., pages 67-82. EDP Sci., Les Ulis, 2008.

38. M. Á. Fernández and M. Moubachir. An exact block-Newton algorithm for solving fluid-structure interaction problems. C. R. Math. Acad. Sci. Paris, 336(8):681-686, 2003.

39. M. Á. Fernández and M. Moubachir. A Newton method using exact jacobians for solving fluid-structure coupling. Computers \& Structures, 83:127-142, 2005.

40. A. Fischle. A Parallel Newton-Krylov-FETI-DP solver based on FEAP. PhD thesis, Fakultät für Mathematik, Universität Duisburg-Essen, 2015.

41. A. Fischle, A. Heinlein, A. Klawonn, and O. Rheinbach. Lightweight coupling library for FEAP and LifeV. 2014.

42. L. Formaggia, M. Fernandez, A. Gauthier, J. Gerbeau, C. Prud'homme, and A. Veneziani. The LifeV Project. Web, 2002-2004.

43. L. Formaggia, A. Quarteroni, and A. Veneziani, editors. Cardiovascular mathematics, volume 1 of MSESA. Modeling, Simulation and Applications. Springer-Verlag Italia, Milan, 2009. Modeling and simulation of the circulatory system.

44. M. W. Gee, C. Förster, and W. A. Wall. A computational strategy for prestressing patient-specific biomechanical problems under finite deformation. Int. J. Numer. Methods Biomed. Eng., 26:52-72, 2010.

45. M. W. Gee, U. Küttler, and W. A. Wall. Truly monolithic algebraic multigrid for fluidstructure interaction. Internat. J. Numer. Methods Engrg., 26:52-72, 2010.

46. J.-F. Gerbeau and M. Vidrascu. A quasi-Newton algorithm based on a reduced model for fluid-structure interaction problems in blood flows. M2AN Math. Model. Numer. Anal., 37(4):631-647, 2003.

47. G. Holzapfel and T. Gasser. A viscoelastic model for fiber-reinforced composites at finite strains: continuum basis, computational aspects and applications. Comput. Methods Appl. Mech. Engrg., 190:4379-4403, 2001. 
48. G. Holzapfel, T. Gasser, and R. Ogden. A new constitutive framework for arterial wall mechanics and a comparative study of material models. Journal of Elasticity, 61:1-48, 2000.

49. J. Hron and S. Turek. A monolithic fem/multigrid solver for an ALE formulation of fluid-structure interaction with applications in biomechanics. In H.-J. Bungartz and M. Schäfer, editors, Fluid-structure interaction, volume 53 of Lecture Notes in Computational Science and Engineering, pages 146-170, 2006.

50. B. Hübner, E. Walhorn, and D. Dinkler. A monolithic approach to fluid-structure interaction using space-time finite elements. Comput. Methods Appl. Mech. Engrg., 193:2087-2104, 2004.

51. M. Itskov and A. E. Ehret. A universal model for the elastic, inelastic and active behaviour of soft biological tissues. GAMM-Mitteilungen, 32(2):221-236, 2009.

52. B. Janssen and T. Wick. Block preconditioning with Schur complements for monolithic fluid-structure interactions. In Proceedings of V European Conference on Computational Fluid Dynamics ECCOMAS CFD 2010, Lisbon, Portugal,, June 14-17 2010.

53. A. Klawonn and O. Rheinbach. Highly scalable parallel domain decomposition methods with an application to biomechanics. ZAMM Z. Angew. Math. Mech., 90(1):5-32, 2010.

54. U. Küttler, M. W. Gee, C. Förster, A. Comerford, and W. A. Wall. Coupling strategies for biomedical fluid-structure interaction problems. Int. J. Numer. Methods Biomed. Eng., 26(3-4):305-321, 2010.

55. U. Langer and H. Yang. Domain decomposition solvers for some fluid-structure interaction problems. PAMM, 12(1):375$376,2012$.

56. U. Langer and H. Yang. Partitioned solution algorithms for fluid-structure interaction problems with hyperelastic models. J. Comput. Appl. Math., 276:47-61, 2015.

57. M. Mayr, T. Klöppel, W. A. Wall, and M. W. Gee. A temporal consistent monolithic approach to fluid-structure interaction enabling single field predictors. SIAM J. Sci. Comput., 37(1):B30-B59, 2015.

58. M. F. Murphy, G. H. Golub, and A. J. Wathen. A note on preconditioning for indefinite linear systems. SIAM J. Sci. Comput., 21(6):1969-1972 (electronic), 2000.

59. F. Nobile and C. Vergara. An effective fluid-structure interaction formulation for vascular dynamics by generalized Robin conditions. SIAM J. Sci. Comput., 30(2):731-763, 2008.

60. S. Patankar and D. Spalding. A calculation procedure for heat, mass and momentum transfer in three dimensional parabolic flows. International J. on Heat and Mass Transfer, 15:1787-1806, 1972.

61. C. S. Peskin. The immersed boundary method. Acta Numerica, 11:479-517, 2002.

62. A. Quarteroni, F. Saleri, and A. Veneziani. Factorization methods for the numerical approximation of Navier-Stokes equations. Comput. Methods Appl. Mech. Engrg., 188(1-3):505-526, 2000.

63. R. Rannacher and T. Richter. An adaptive finite element method for fluid-structure interaction problems based on a fully eulerian formulation. In H.-J. B. et al., editor, Fluid Structure Interaction II, pages 159-192. Springer-Verlag Berlin Heidelberg, 2010. Lecture Notes in Computational Science and Engineering 73.

64. M. Razzaq, H. Damanik, J. Hron, A. Ouazzi, and S. Turek. FEM multigrid techniques for fluid-structure interaction with application to hemodynamics. Appl. Numer. Math., 62(9):1156-1170, 2012.

65. M. Razzaq, J. Hron, and S. Turek. Numerical simulation of laminar incompressible fluid-structure interaction for elastic material with point constraints. In Advances in mathematical fluid mechanics, pages 451-472. Springer, Berlin, 2010.

66. O. Rheinbach. Parallel iterative substructuring in structural mechanics. Arch. Comput. Methods Eng., 16(4):425-463, 2009.

67. O. Rheinbach, A. Klawonn, J. Schröder, D. Balzani, and D. Brands. Simulation of arterial walls: an algebraic interface to iterative substructuring. Proceedings of ICCB 2007 - International Congress on Computational Bioengineering, 2007.

68. T. Richter. A fully eulerian formulation for fluid-structure-interaction problems with large deformations and free structure movement. In Proceedings of the $V$ European Conference on Computational Fluid Dynamics ECCOMAS CFD 2010, Lisbon, Portugal, 14-17 June 2010, 2010.

69. P. A. Sackinger, P. R. Schunk, and R. R. Rao. A Newton-Raphson pseudo-solid domain mapping technique for free and moving boundary problems: a finite element implementation. J. Comput. Phys., 125(1):83-103, 1996.

70. C. Sansour. On the physical assumptions underlying the volumetric-isochoric split and the case of anisotropy. European Journal of Mechanics A/Solids, 27:28-39, 2008.

71. J. Schröder and P. Neff. Invariant formulation of hyperelastic transverse isotropy based on polyconvex free energy functions. Internat. J. Solids Structures, 40:401-445, 2003.

72. J. Schröder, P. Neff, and D. Balzani. A variational approach for materially stable anisotropic hyperelasticity. Internat. J. Solids Structures, 42(15):4352-4371, 2005.

73. J. Simo. On a fully three-dimensional finite-strain viscoelastic damage model: formulation and computational aspects. Comput. Methods Appl. Mech. Engrg, 60:153-173, 1987.

74. J. Simo. Numerical analysis and simulation of plasticity. In P. Ciarlet and J. Lions, editors, Handbook of numerical analysis, number 6. Elsevier Science, 1998.

75. B. Smith, P. Bjørstad, and W. Gropp. Domain Decomposition: Parallel Multilevel Methods for Elliptic Partial Differential Equations. Cambridge University Press, 1996.

76. M. Tanaka, M. Fujikawa, D. Balzani, and J. Schröder. Robust numerical calculation of tangent moduli at finite strains based on complex-step derivative approximation and its application to localization analysis. Comput. Methods Appl. Mech. Engrg., 269:454-470, 2014. 
77. R. Taylor. FEAP - a finite element analysis program. http://www.ce.berkeley.edu/ rlt/feap/.

78. T. E. Tezduyar, S. Sathe, and K. Stein. Solution techniques for the fully discretized equations in computation of fluidstructure interactions with the space-time formulations. Comput. Methods Appl. Mech. Engrg., 195:5743-5753, 2006.

79. P. Tricerri, L. Dedè, S. Deparis, A. Quarteroni, A. Robertson, and A. Sequeira. Fluid-structure interaction simulations of cerebral arteries modeled by isotropic and anisotropic constitutive laws. Comput. Mech., 55(3):479-498, 2015.

80. S. Turek, J. Hron, M. Razzaq, H. Wobker, and M. Schäfer. Numerical benchmarking of fluid-structure interaction: A comparison of different discretization and solution approaches. In H.-J. Bungartz, M. Mehl, and M. Schäfer, editors, Fluid Structure Interaction II, volume 73 of Lecture Notes in Computational Science and Engineering, pages $413-424$. Springer Berlin Heidelberg, 2010.

81. M. ur Rehman, C. Vuik, and G. Segal. A comparison of preconditioners for incompressible Navier-Stokes solvers. Internat. J. Numer. Methods Fluids, 57(12):1731-1751, 2008.

82. W. A. Wall, A. Gerstenberger, U. Küttler, and U. M. Mayer. An XFEM based fixed-grid approach for 3d fluid-structure interaction. In H.-J. B. et al., editor, Fluid Structure Interaction II, pages 327-349. Springer-Verlag Berlin Heidelberg, 2010. Lecture Notes in Computational Science and Engineering 73.

83. H. Wang, J. Chessa, W. K. Liu, and T. Belytschko. The immersed/fictitious element method for fluid-structure interaction: volumetric consistency, compressibility and thin members. Internat. J. Numer. Methods Engrg., 74:32-55, 2008.

84. Y. Wu and X.-C. Cai. A fully implicit domain decomposition based ALE framework for three-dimensional fluid-structure interaction with application in blood flow computation. J. Comput. Phys., 258:524-537, 2014.

85. H. Yang and W. Zulehner. A Newton based fluid-structure interaction solver with algebraic multigrid methods on hybrid meshes. In Domain decomposition methods in science and engineering XIX, volume 78 of Lect. Notes Comput. Sci. Eng., pages 285-292. Springer, Heidelberg, 2011. 


\section{Appendix}

For completeness, this appendix collects systematically the numerical results presented in the text as well as additional graphs.
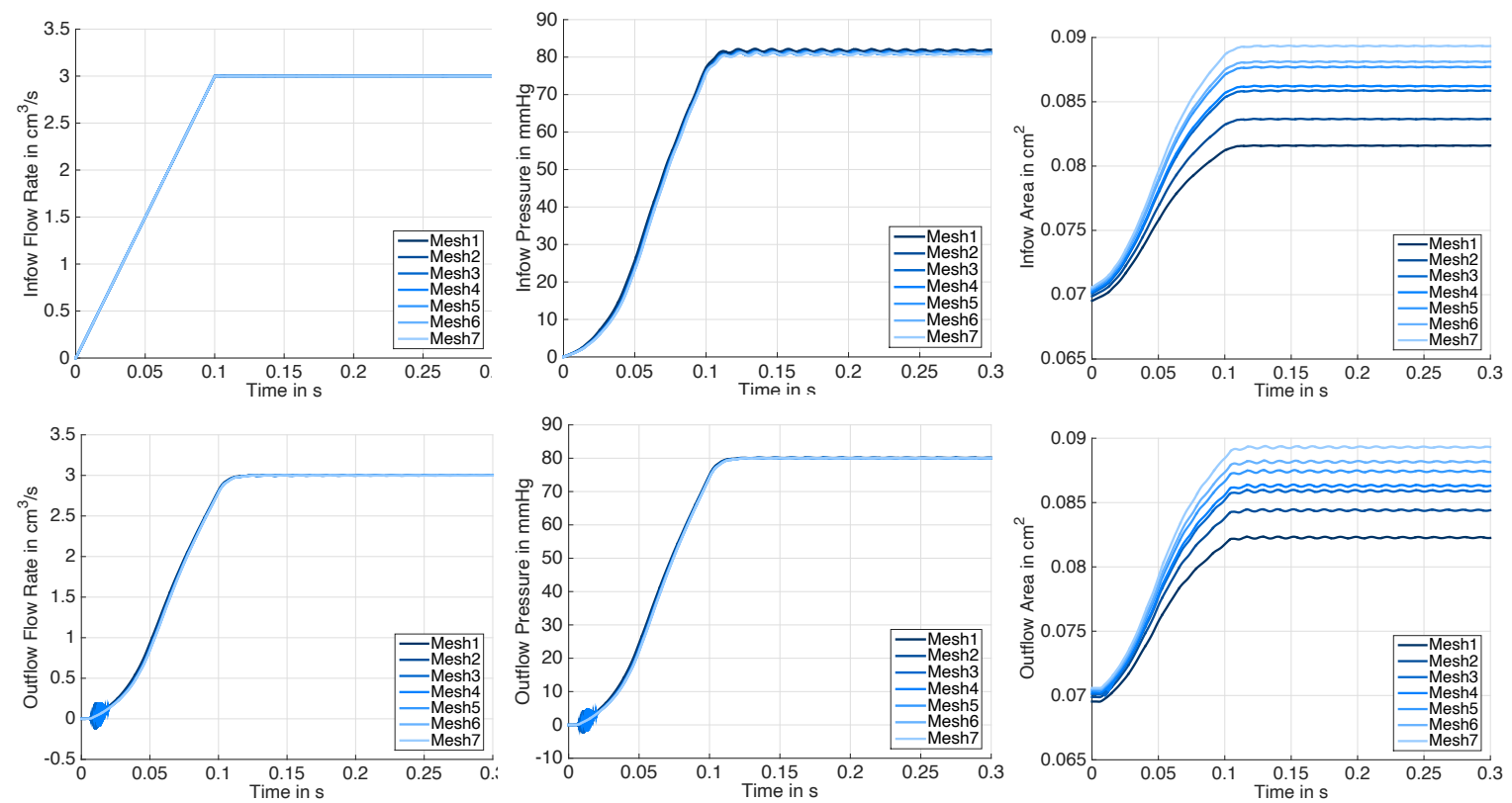

Fig. 26 P1 mesh convergence study for the hyperelastic material using the linear ramp with $T_{\mathrm{R}}=0.1 \mathrm{~s}$. Flowrate (left), average pressure (middle), lumen cross section area (right), over time at the inlet (top) and outlet (bottom); cf. Section 4.2.1. 

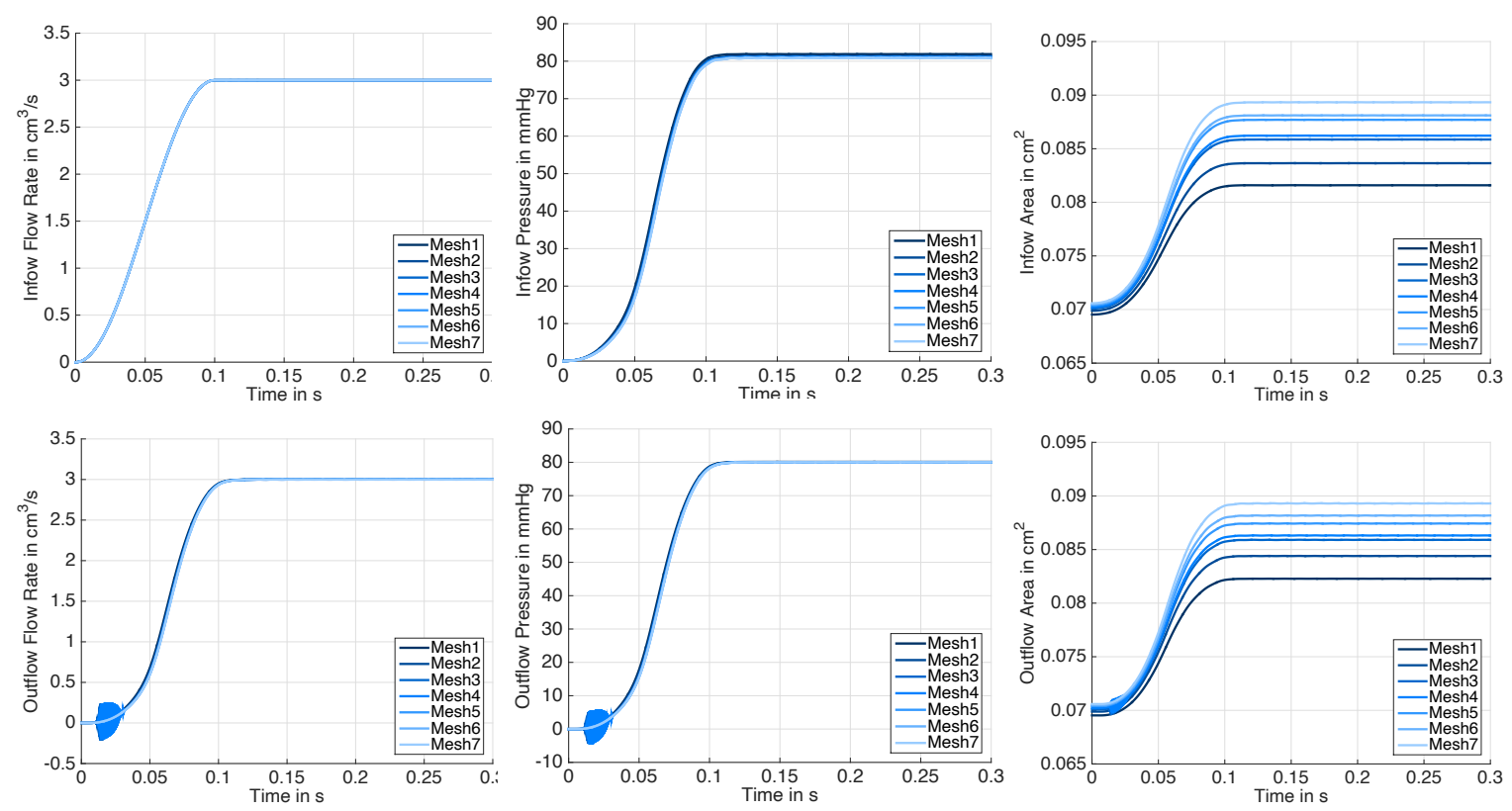

Fig. 27 P1 mesh convergence study for the hyperelastic material using the cosine-type ramp with $T_{\mathrm{R}}=0.1 \mathrm{~s}$. Flowrate (left), average pressure (middle), Lumen cross section area (right), over time at the inlet (top) and outlet (bottom); cf. Section 4.2.2.
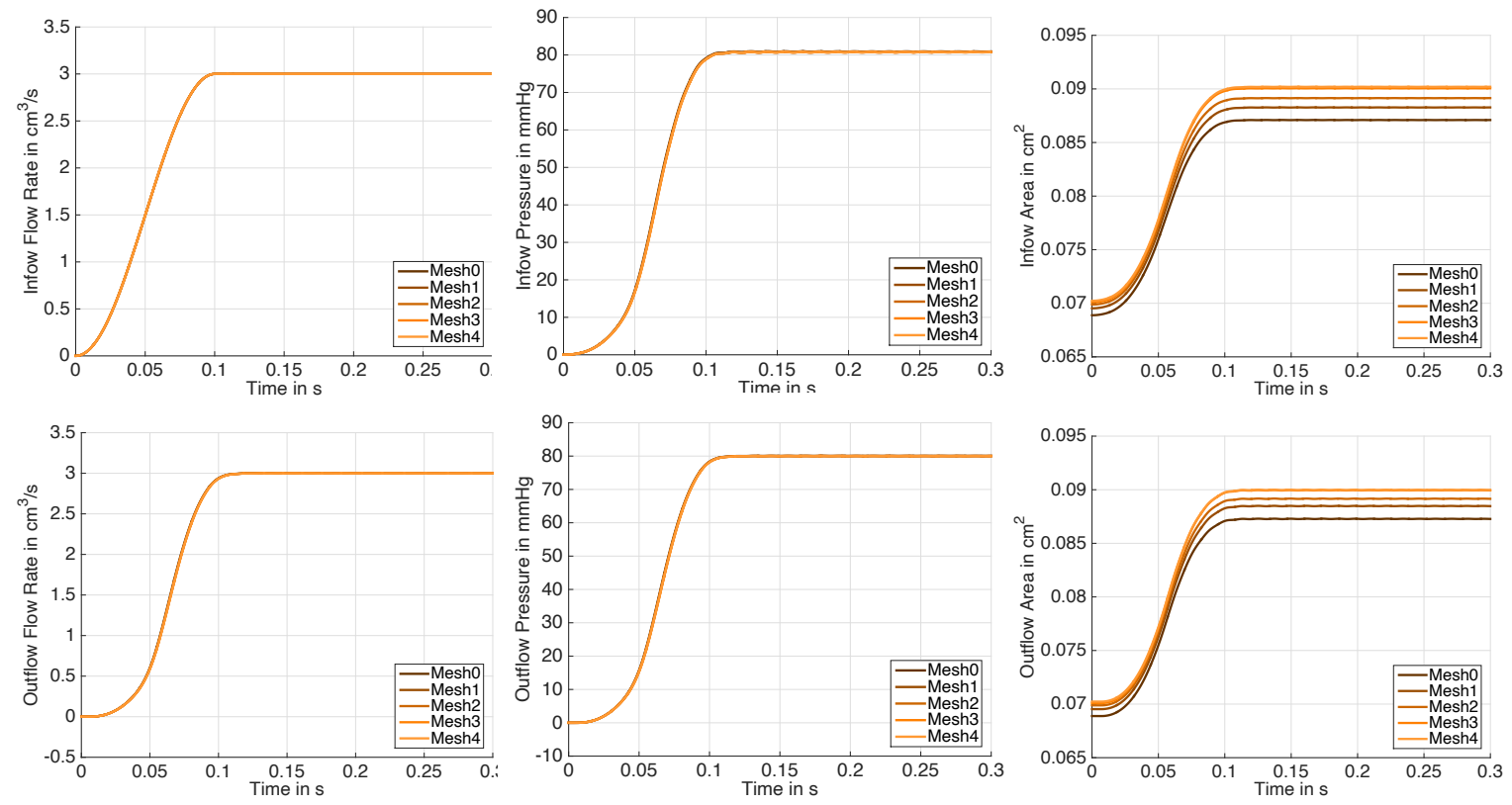

Fig. 28 P2 mesh convergence study for the hyperelastic material using the cosine-type ramp with $T_{\mathrm{R}}=0.1 \mathrm{~s}$. Flowrate (left), average pressure (middle), Lumen cross section area (right), over time at the inlet (top) and outlet (bottom); cf. Section 4.2.2. 

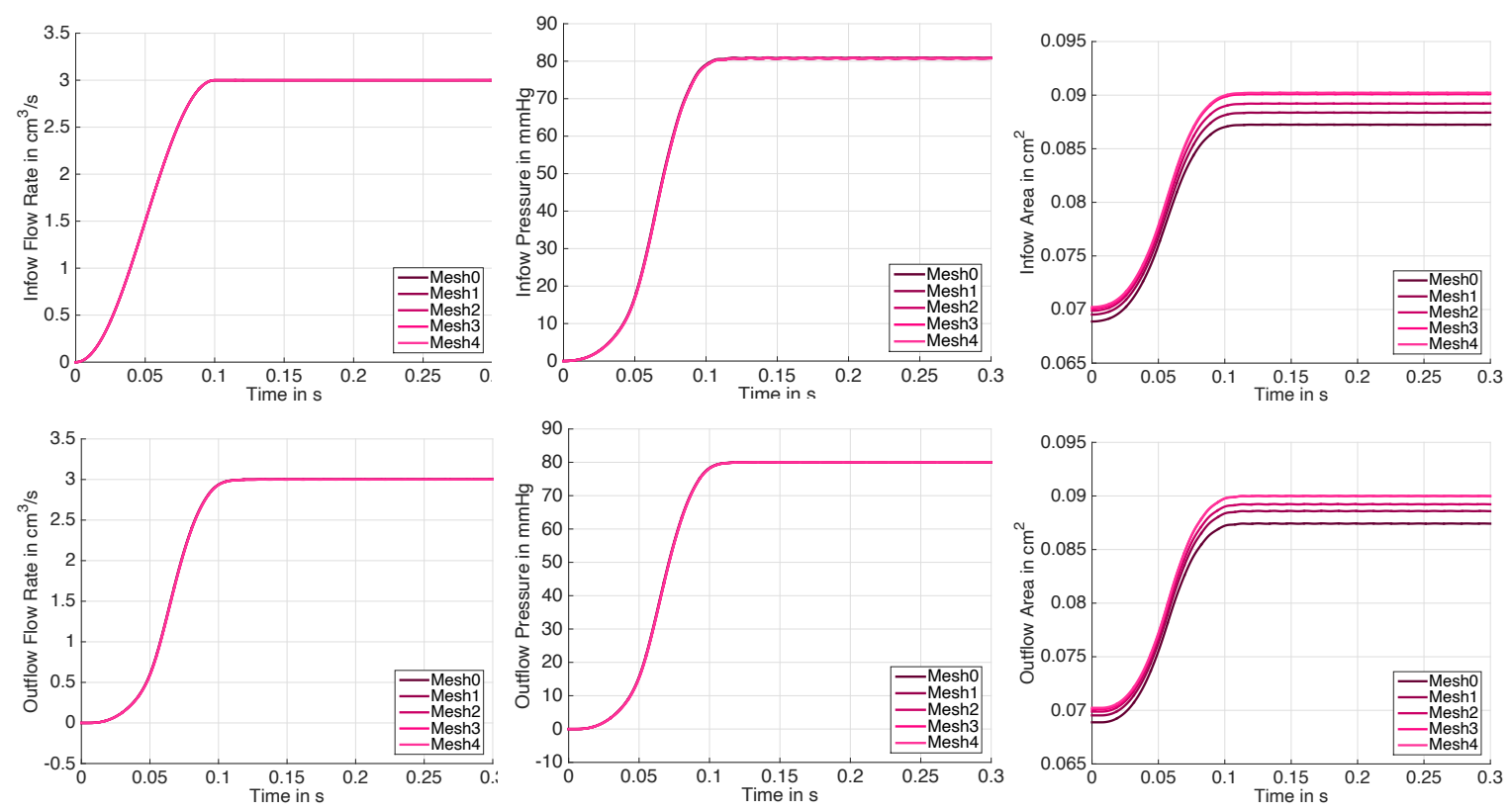

Fig. $29 \overline{\mathrm{F}}$ mesh convergence study for the hyperelastic material using the cosine-type ramp with $T_{\mathrm{R}}=0.1 \mathrm{~s}$. Flowrate (left), average pressure (middle) lumen cross section area (right), over time at the inlet (top) and outlet (bottom); cf. Section 4.2.2.
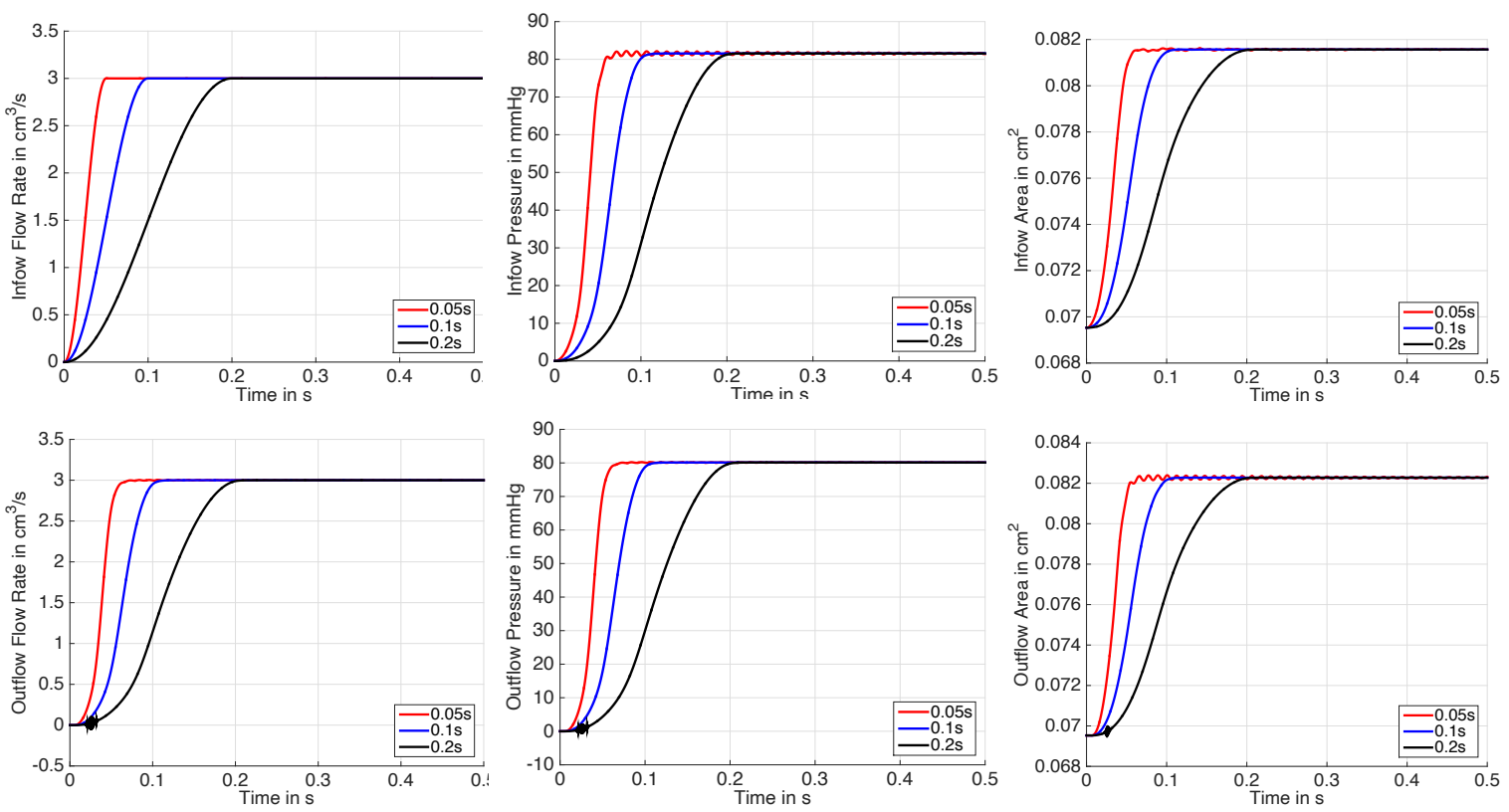

Fig. 30 Hyperelastic material using P1 elements on Mesh \#1. Flowrate (left), average pressure (middle), Lumen cross section area (right), over time at the inlet (top) and outlet (bottom); cf. Section 4.2.3. 

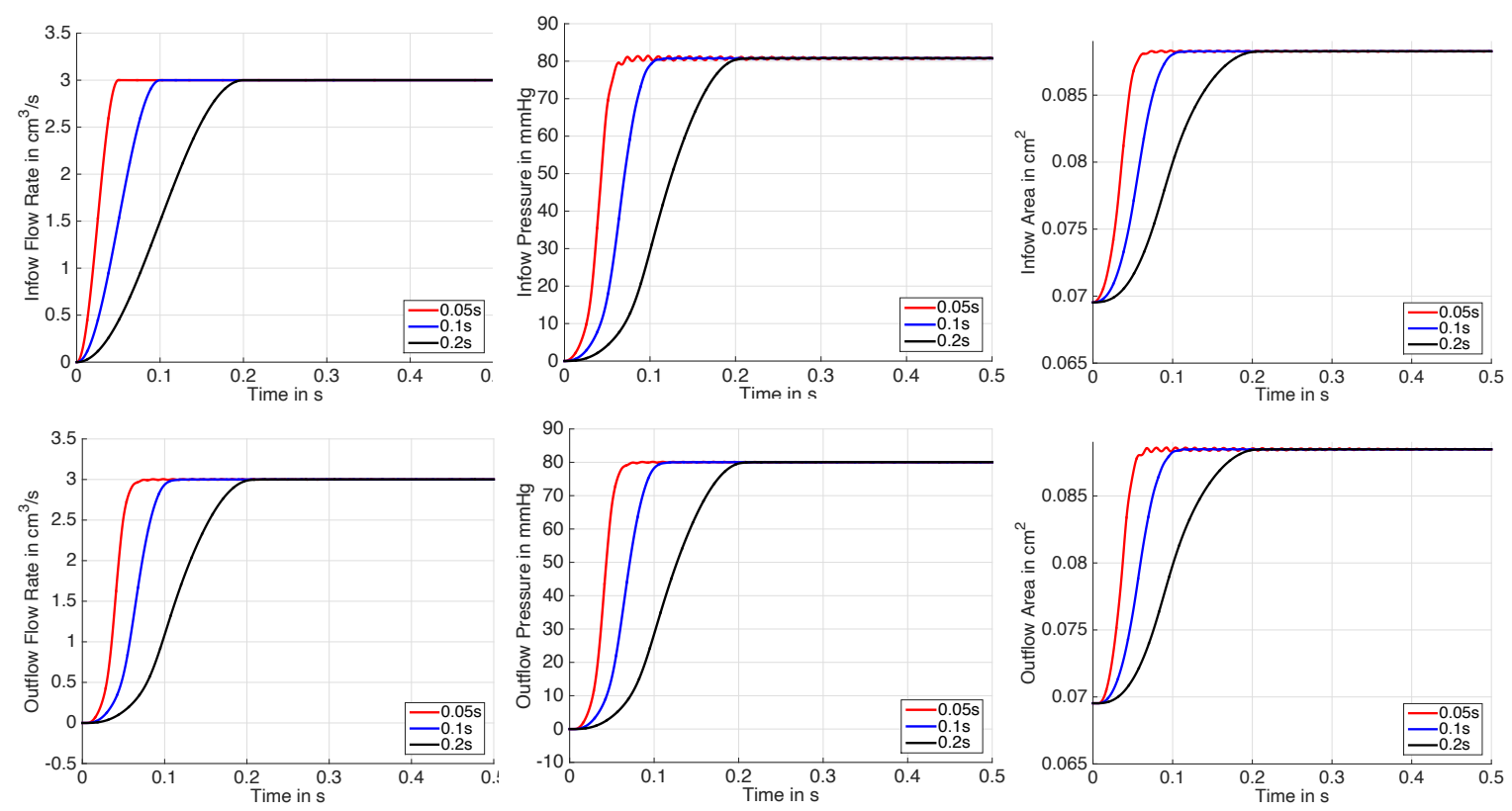

Fig. 31 Hyperelastic material using P2 elements on Mesh \#1. Flowrate (left), average pressure (middle), Lumen cross section area (right), over time at the inlet (top) and outlet (bottom); cf. Section 4.2.3.
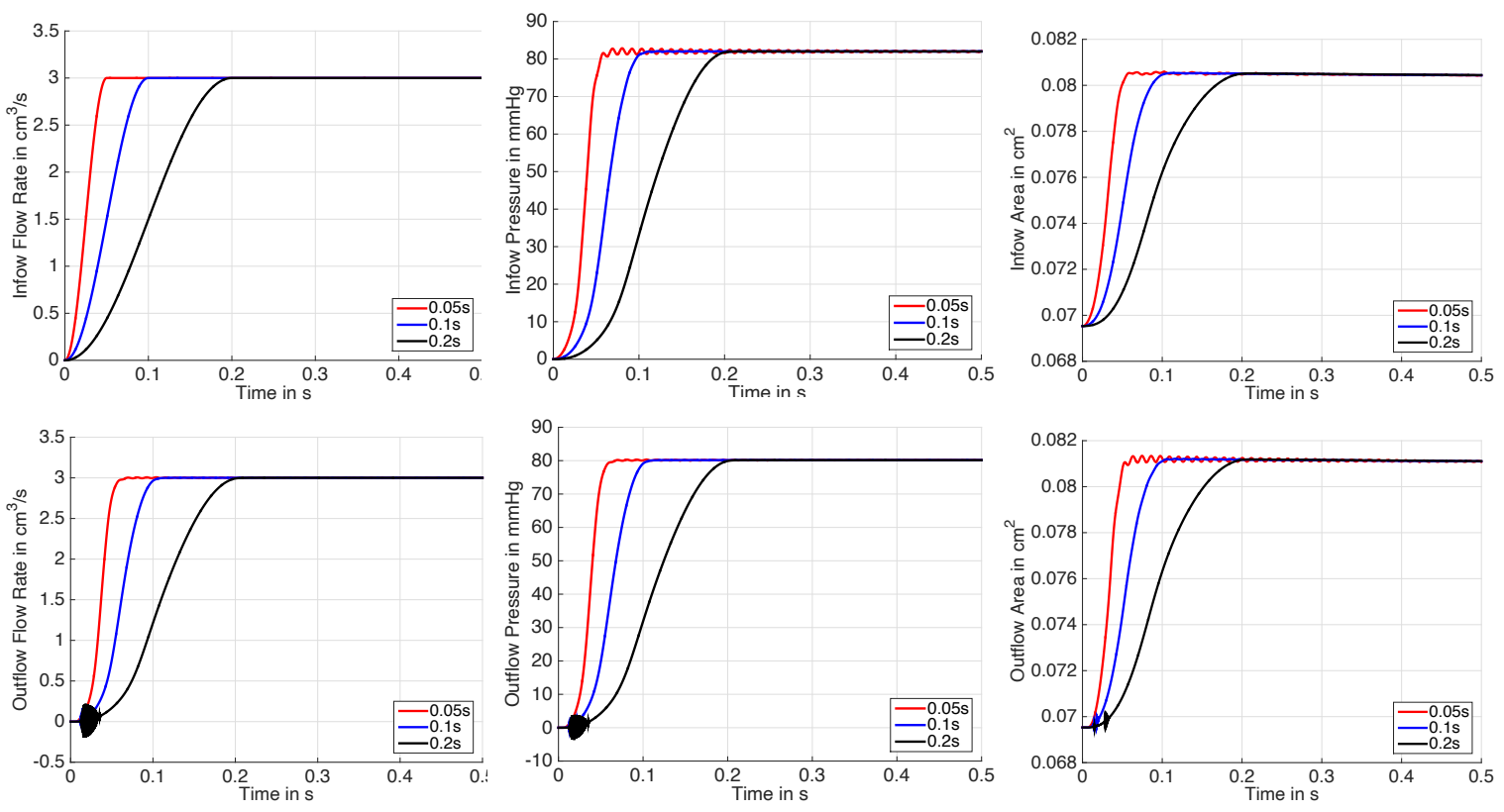

Fig. 32 Viscoelastic material with P1 using the parameter Set 1 from Table 3 elements on Mesh \#1. Flowrate (left), average pressure (middle), Lumen cross section area (right), over time at the inlet (top) and outlet (bottom); cf. Section 4.2.3. 

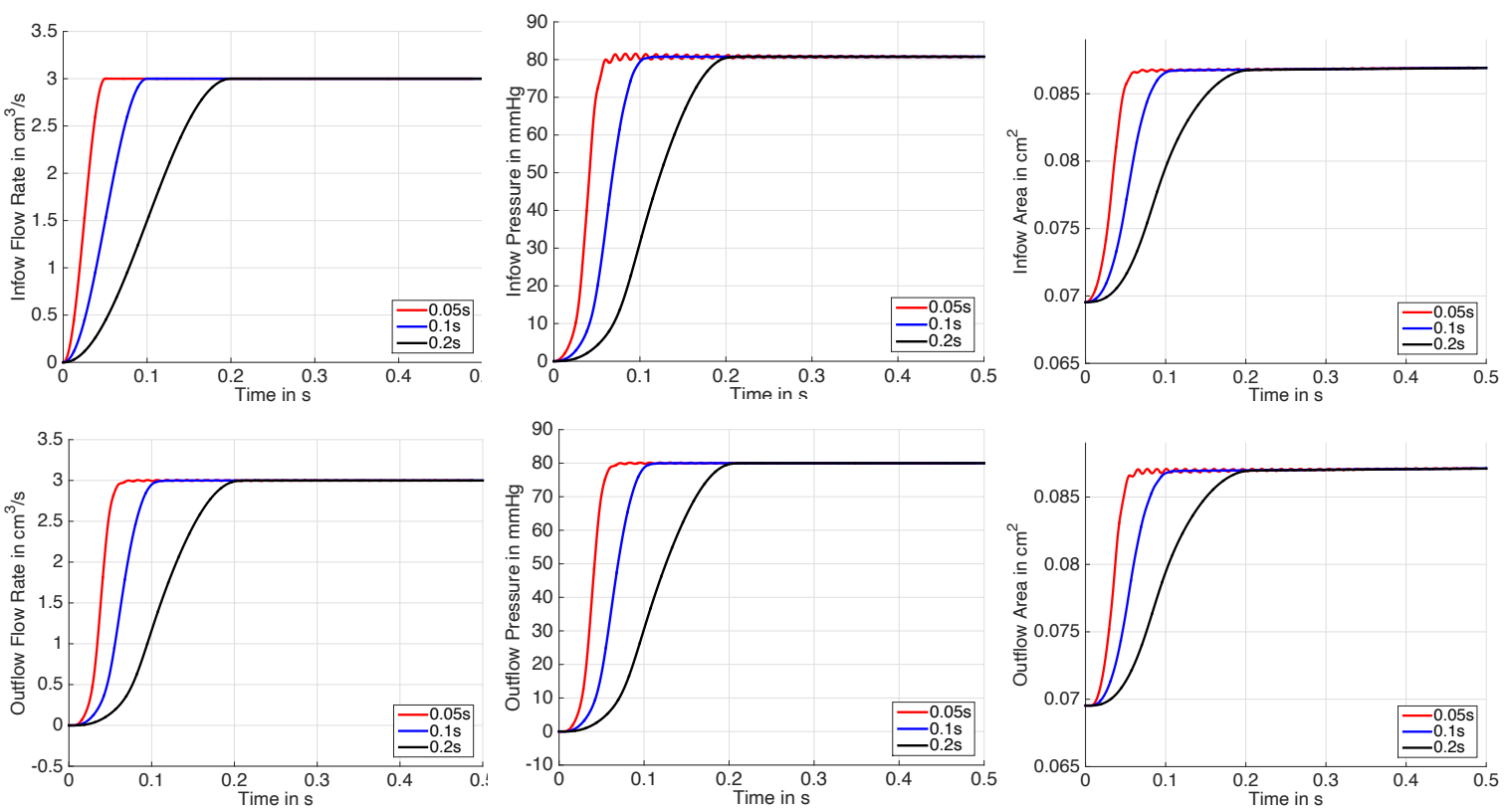

Fig. 33 Viscoelastic material with P2 elements using the parameter Set 1 from Table 3 on Mesh \#1. Flowrate (left), average pressure (middle), Lumen cross section area (right), over time at the inlet (top) and outlet (bottom); cf. Section 4.2.3.
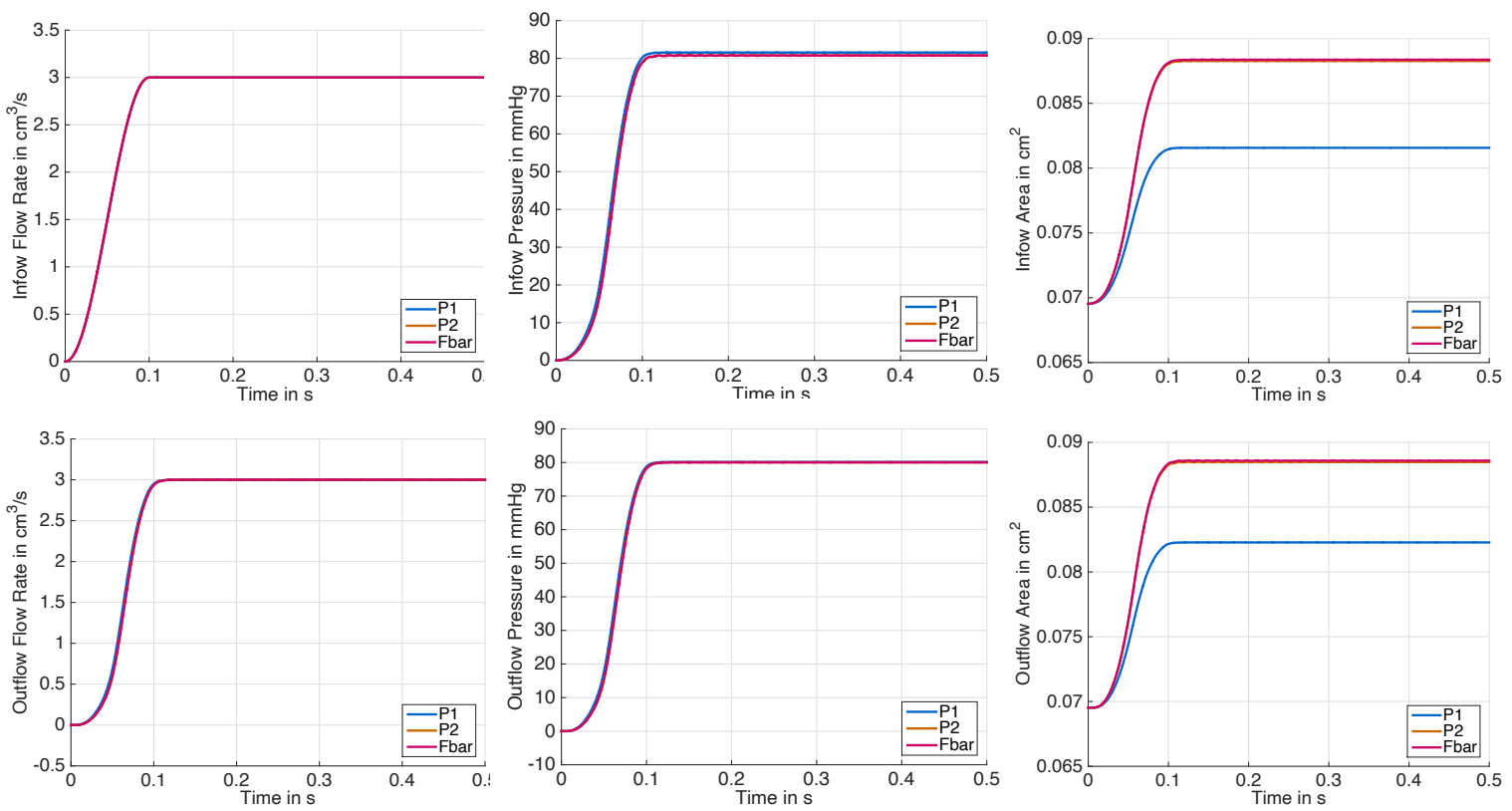

Fig. 34 Hyperelastic material on Mesh \#1. Flowrate (left), average pressure (middle), Lumen cross section area (right), over time at the inlet (top) and outlet (bottom); cf. Section 4.2.4. 

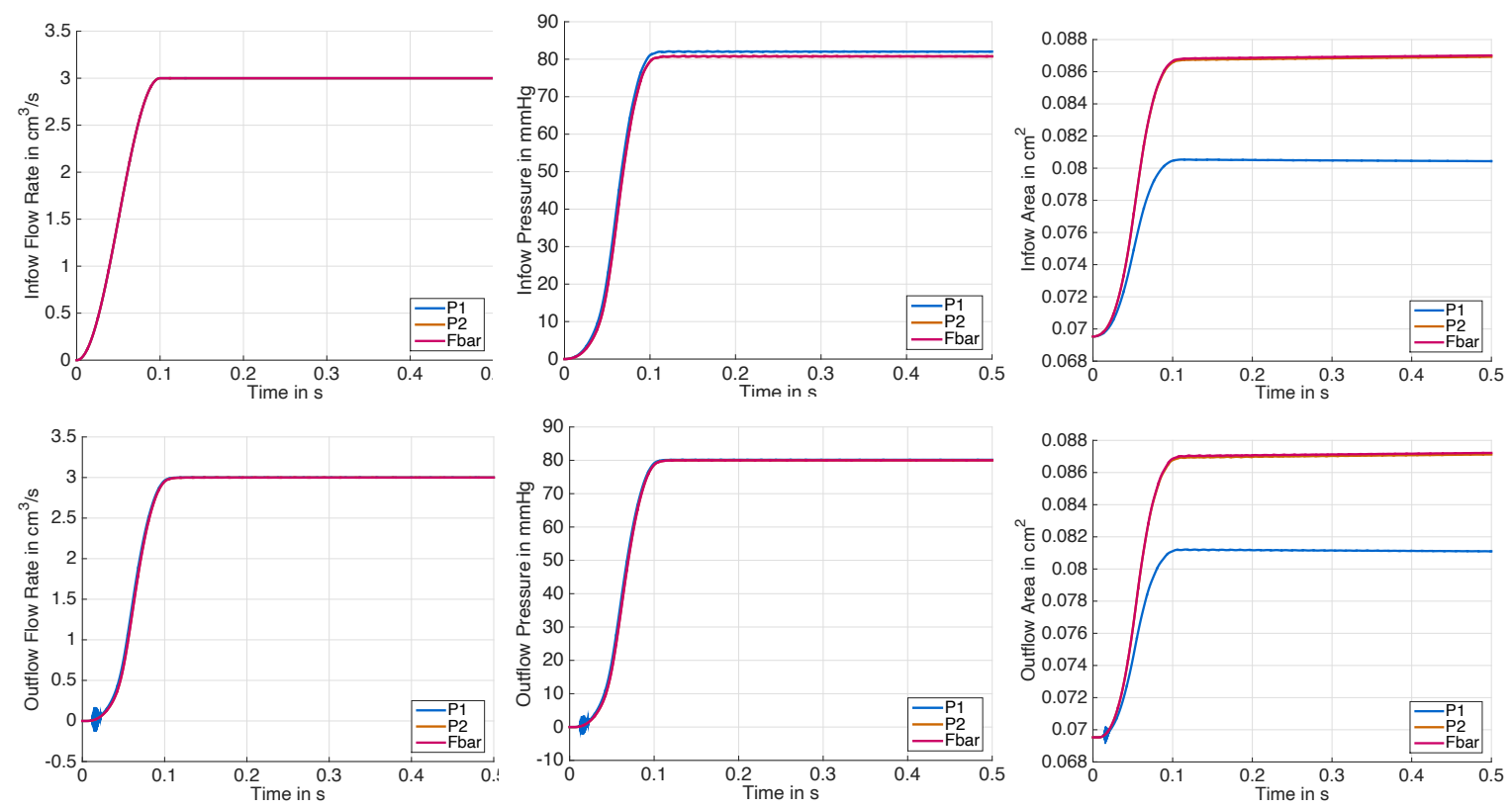

Fig. 35 Viscoelastic material using the parameter Set 1 from Table 3 on Mesh \#1. Flowrate (left), average pressure (middle), Lumen cross section area (right), over time at the inlet (top) and outlet (bottom); cf. Section 4.2.4.
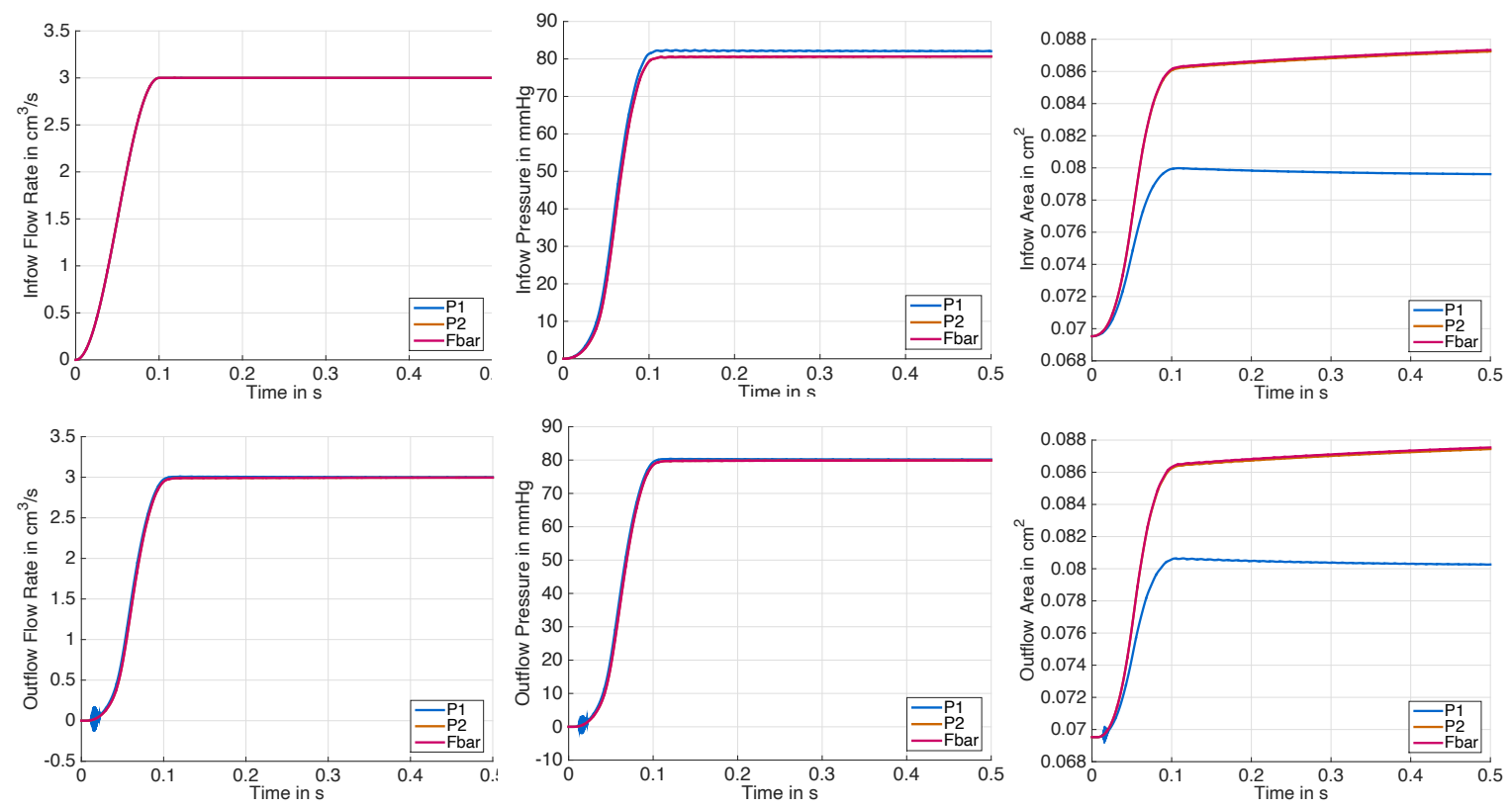

Fig. 36 Viscoelastic material using the parameter Set 2 from Table 3 on Mesh \#1. Flowrate (left), average pressure (middle), Lumen cross section area (right), over time at the inlet (top) and outlet (bottom); cf. Section 4.2.4. 

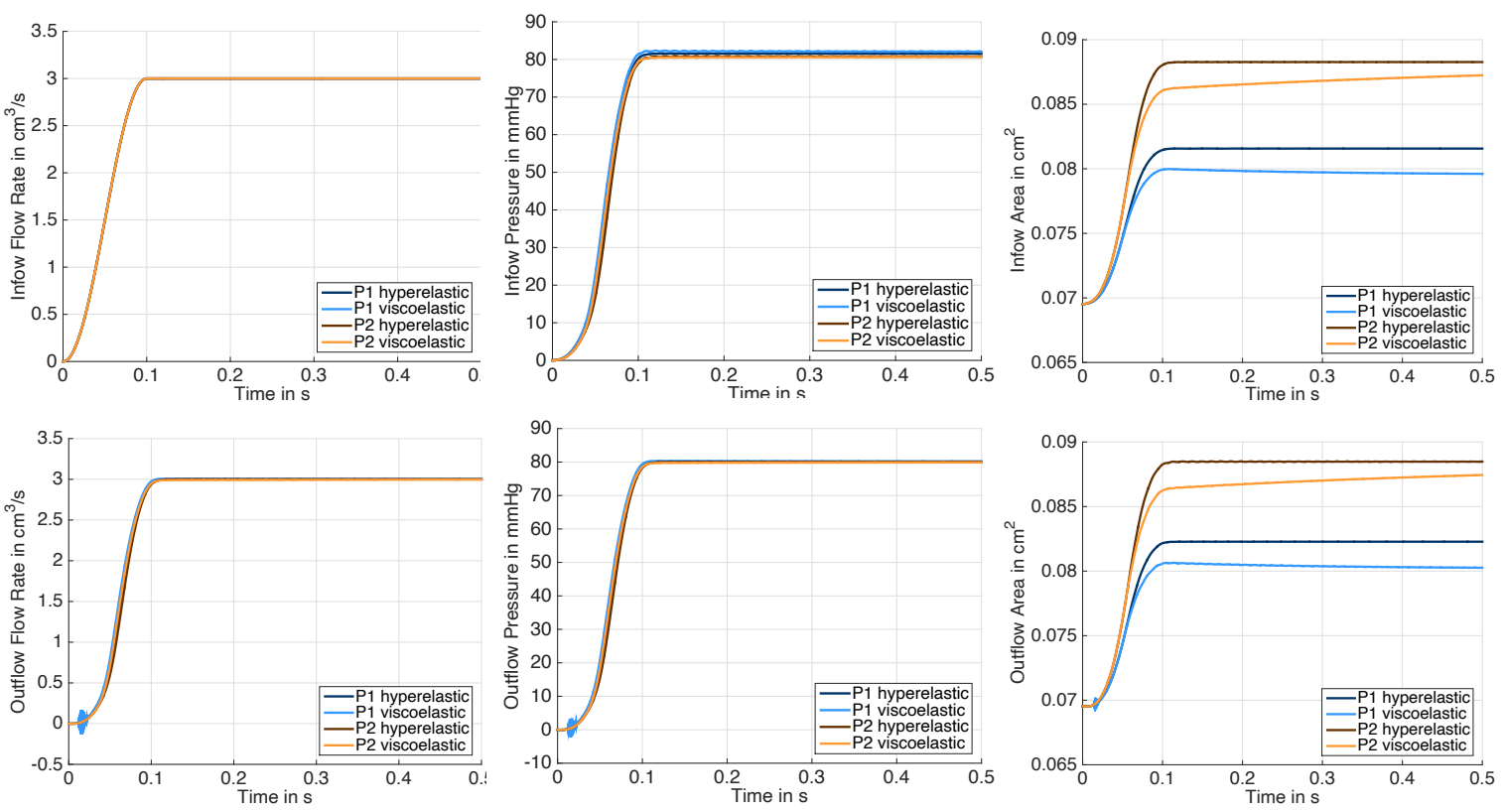

Fig. 37 Comparison of the hyperelastic and the viscoelastic material using the parameter Set 2 from Table 3 on Mesh \#1. Flowrate (left), average pressure (middle), Lumen cross section area (right), over time at the inlet (top) and outlet (bottom); cf. Section 4.2.4.
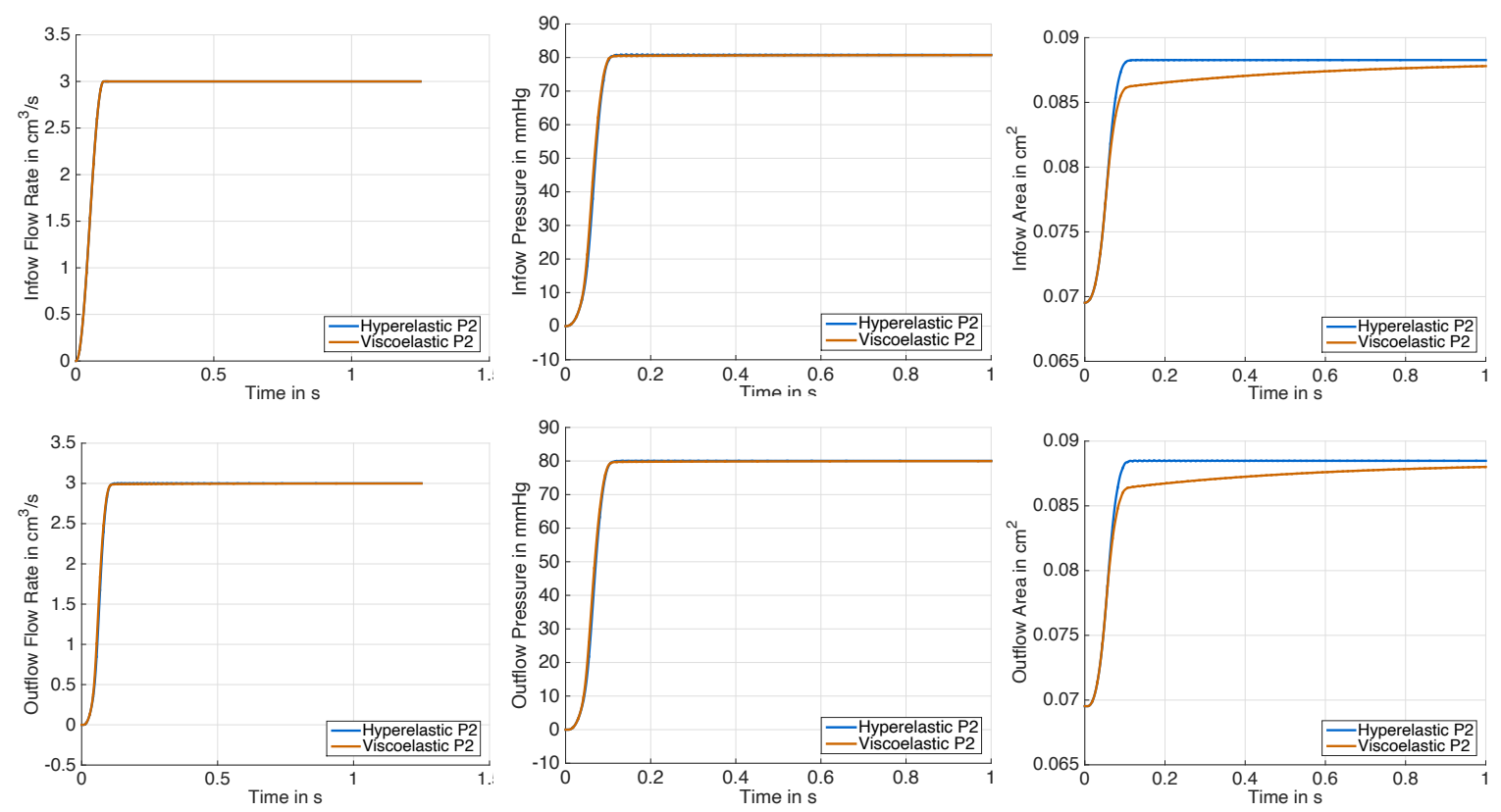

Fig. 38 Comparison of the hyperelastic and the viscoelastic for P2 elements material using the cosine-type ramp and parameter Set 2 from Table 3 on Mesh \# 1. Flowrate (left), average pressure (middle), Lumen cross section area (right), over time at the inlet (top) and outlet (bottom); cf. Section 4.2.4. 

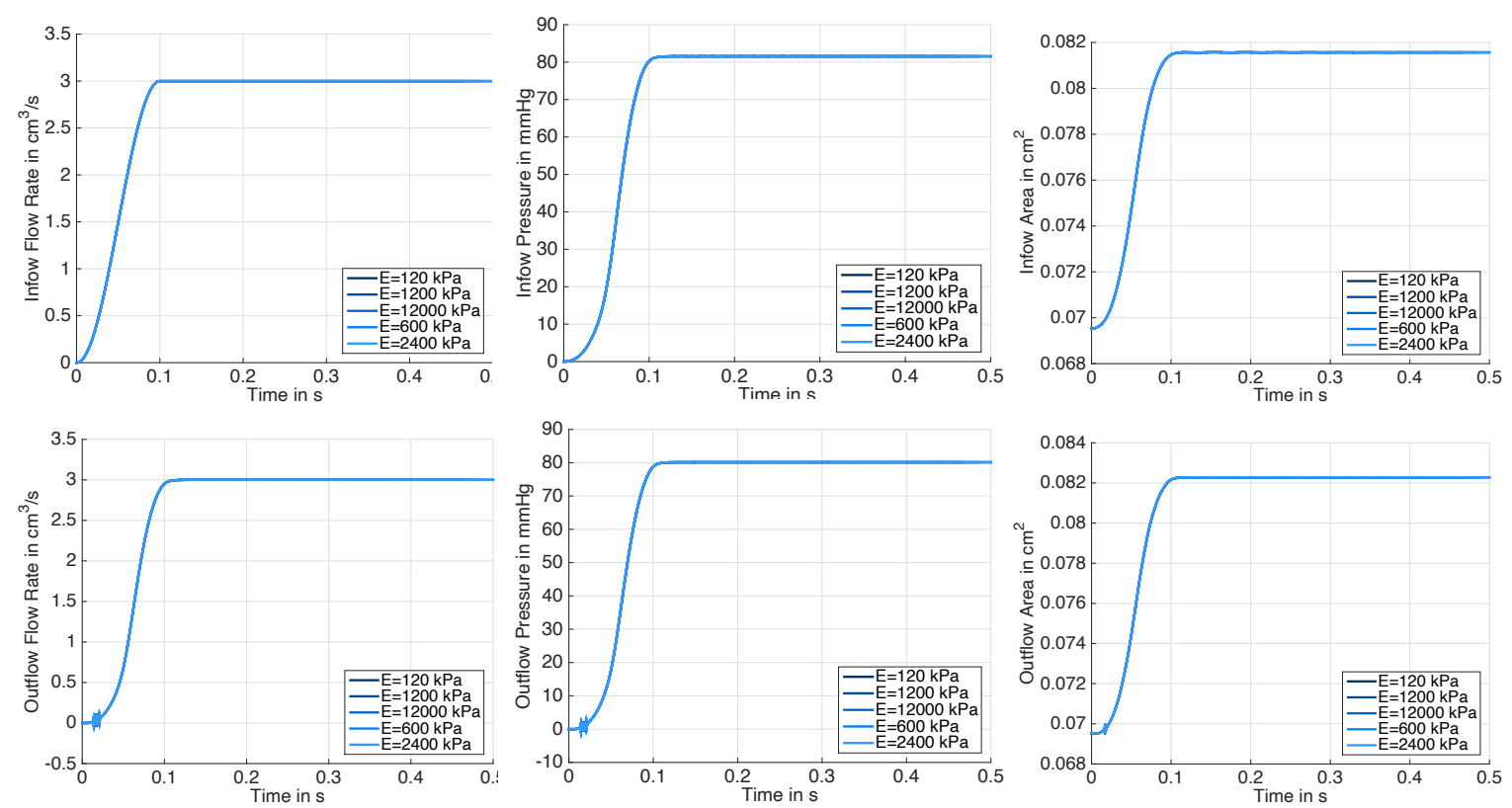

Fig. 39 Sensitivity analysis of the absorbing boundary conditions. Flowrate (left), average pressure (middle), Lumen cross section area (right), over time at the inlet (top) and outlet (bottom); cf. Section 4.2.5.
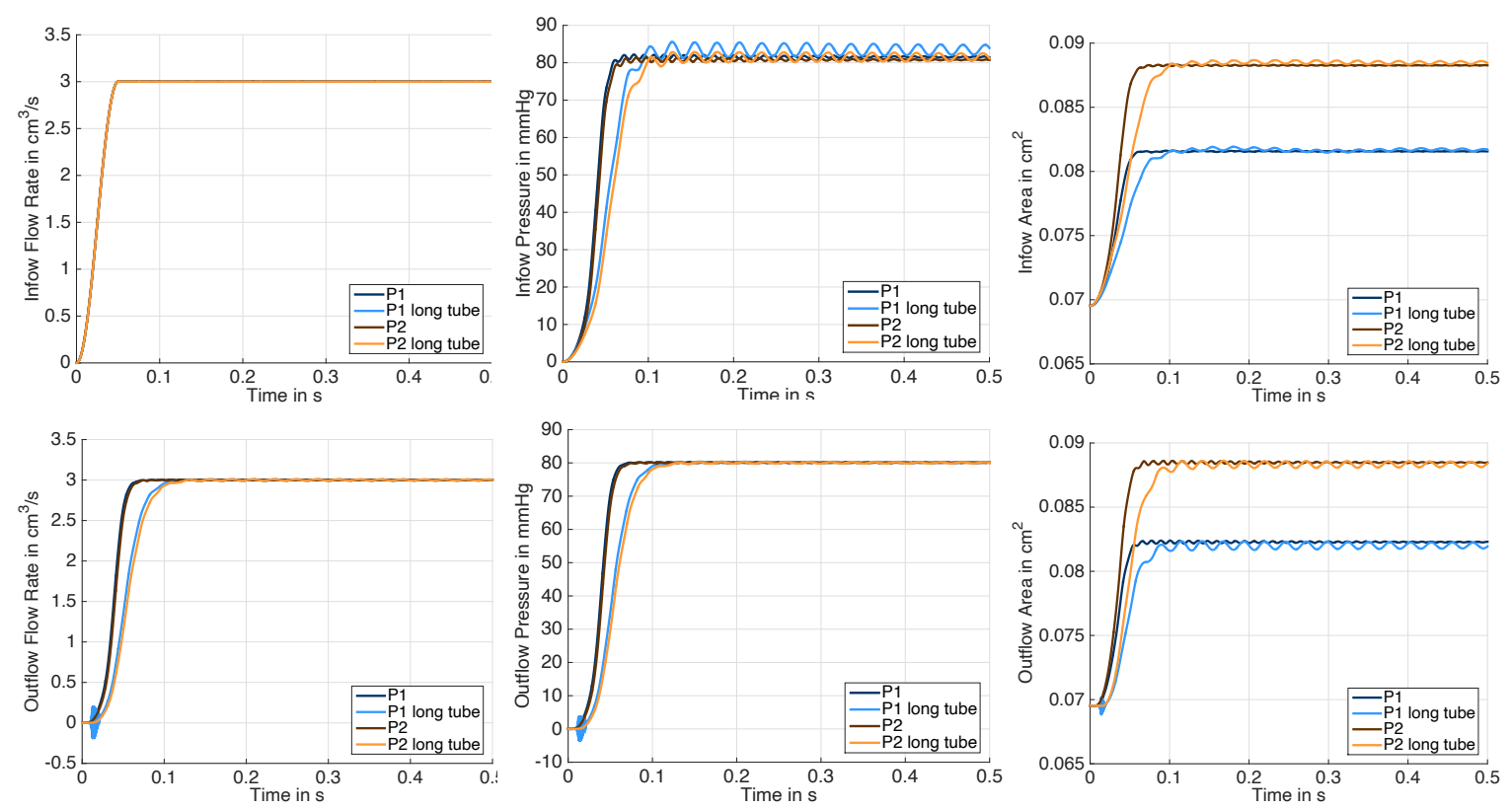

Fig. 40 Comparison of Mesh \#1 and the corresponding mesh of the long tube, cf. Tables 8 and 9. Outflow flow rate (left), inflow average pressure (middle), outflow lumen cross section area (right); cf. Section 4.2.6. 

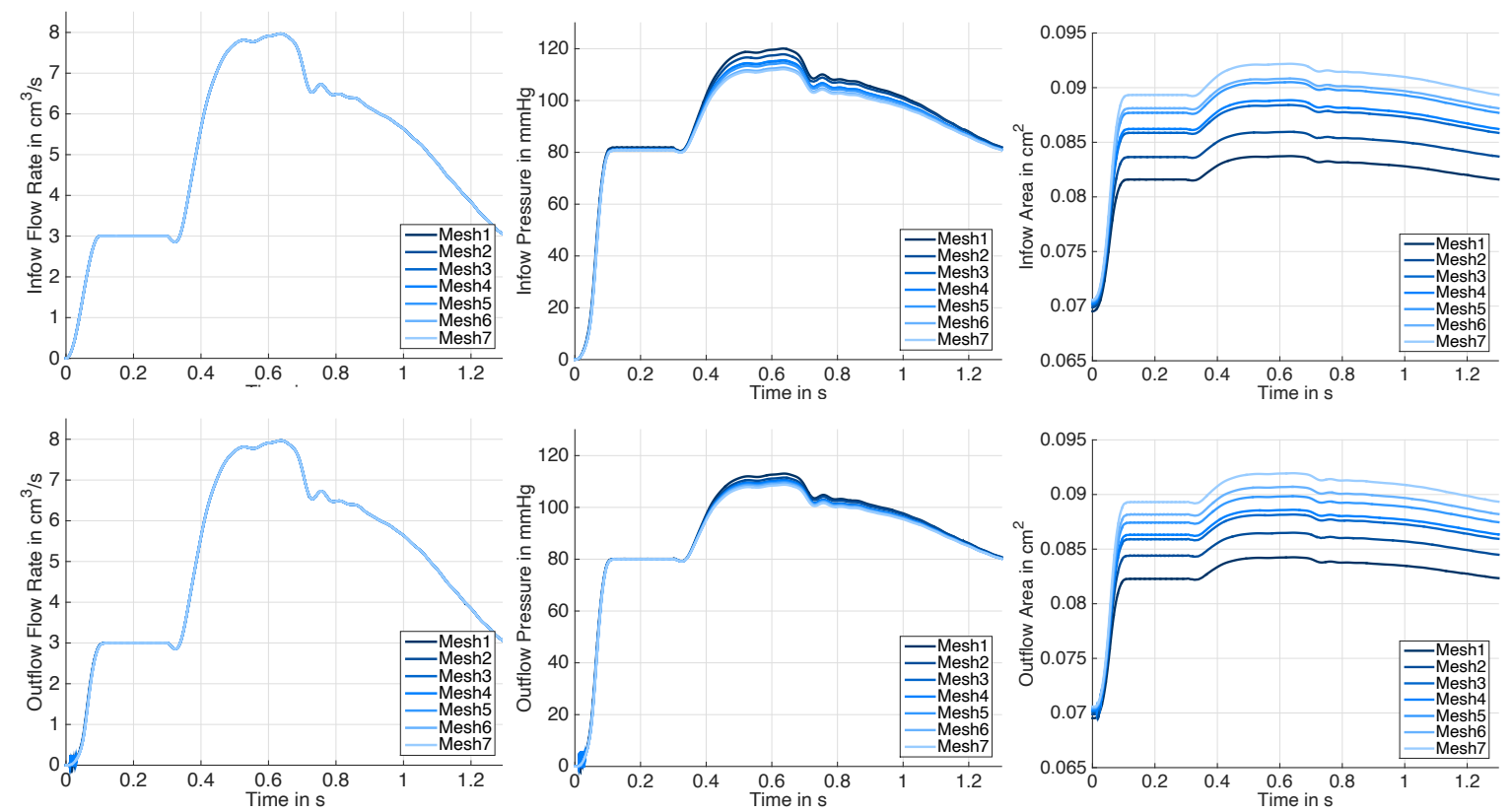

Fig. 41 P1 mesh convergence study for the hyperelastic material for the heartbeat. Flowrate (left), average pressure (middle), Lumen cross section area (right), over time at the inlet (top) and outlet (bottom); cf. Section 4.3.
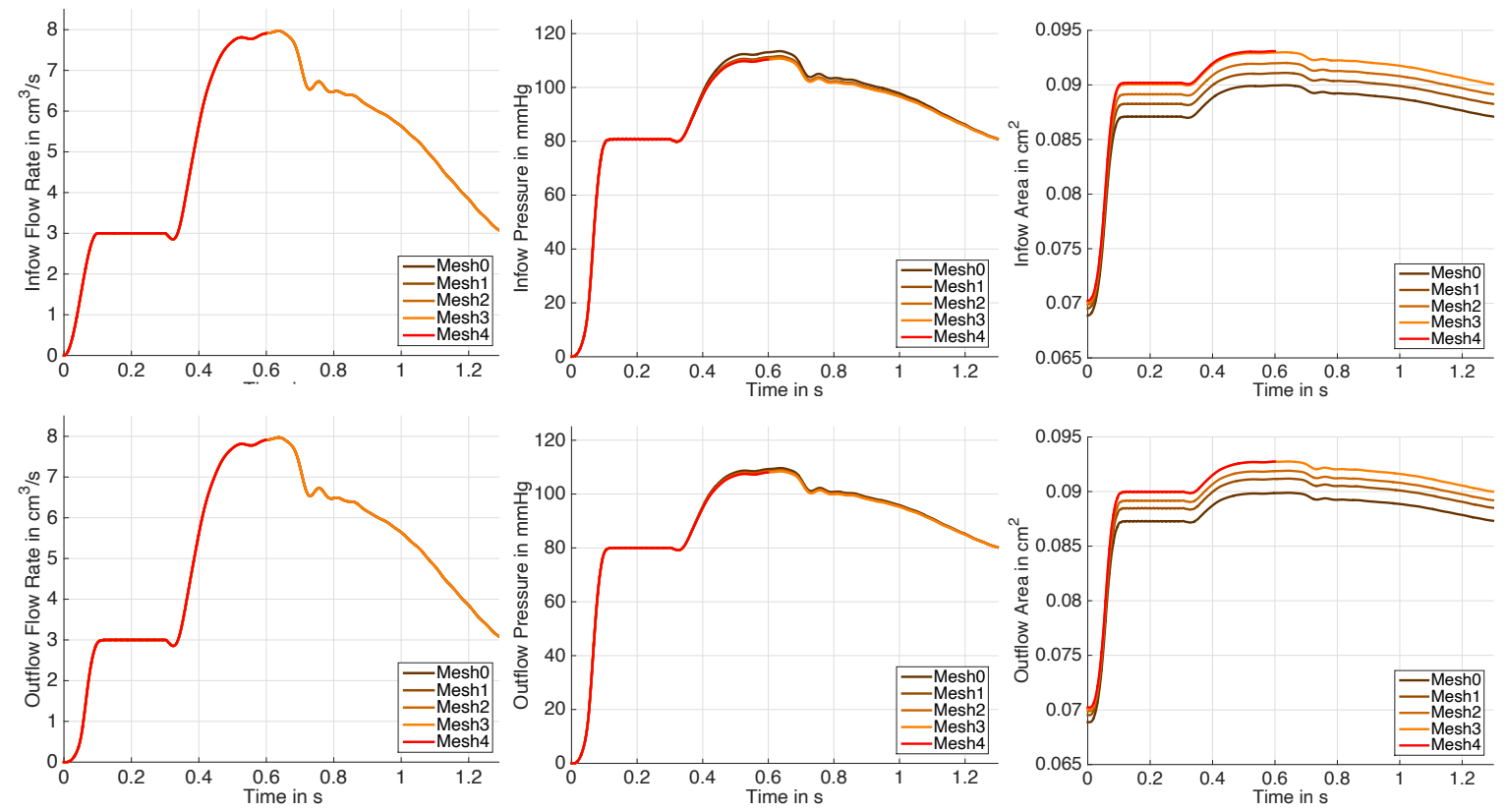

Fig. 42 P2 mesh convergence study for the hyperelastic material for the heartbeat. Flowrate (left), average pressure (middle), Lumen cross section area (right), over time at the inlet (top) and outlet (bottom); cf. Section 4.3. 

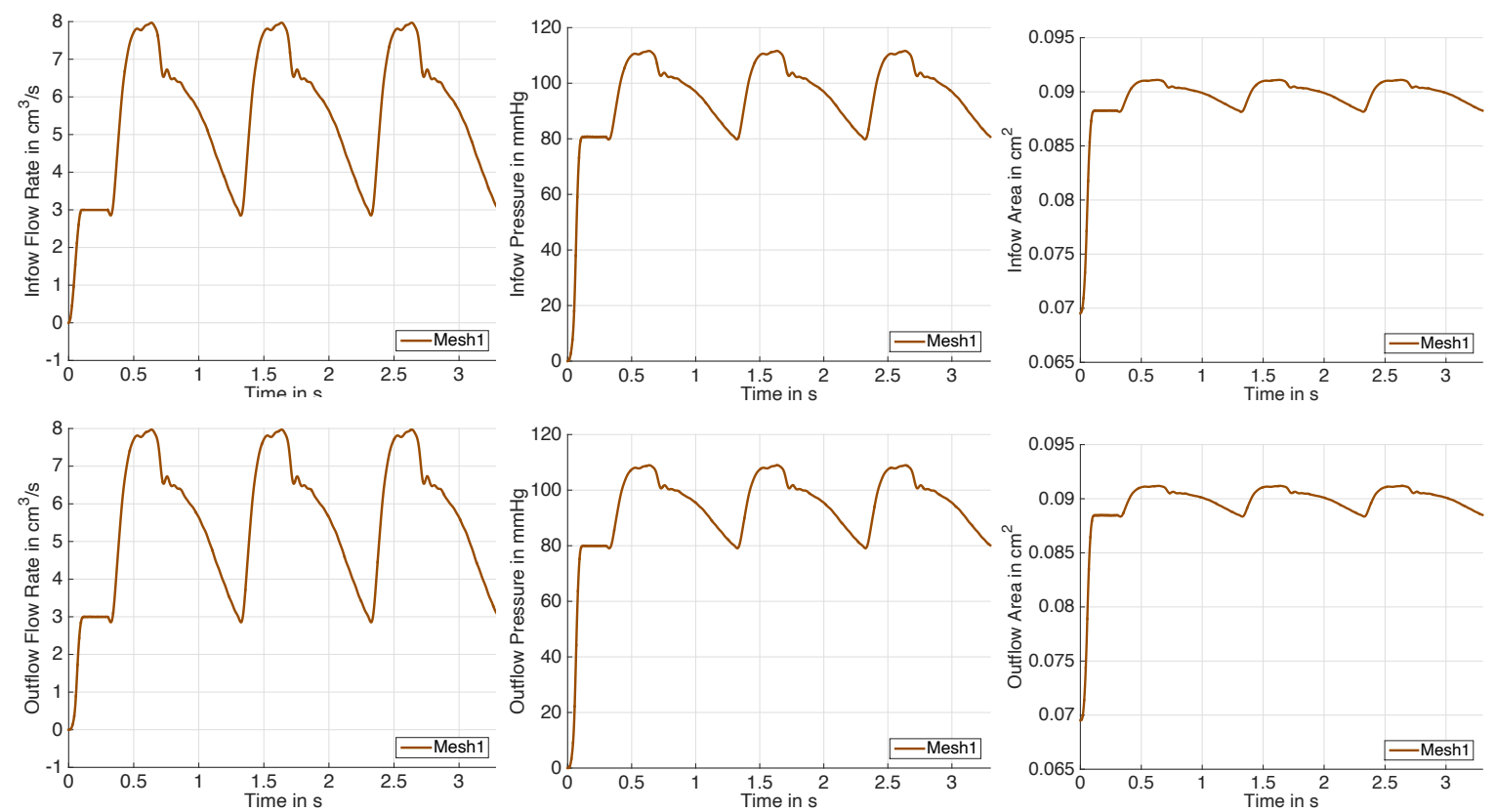

Fig. 43 P2 mesh convergence study for the hyperelastic material for the heartbeat. Flowrate (left), average pressure (middle), Lumen cross section area (right), over time at the inlet (top) and outlet (bottom); cf. Section 4.3.
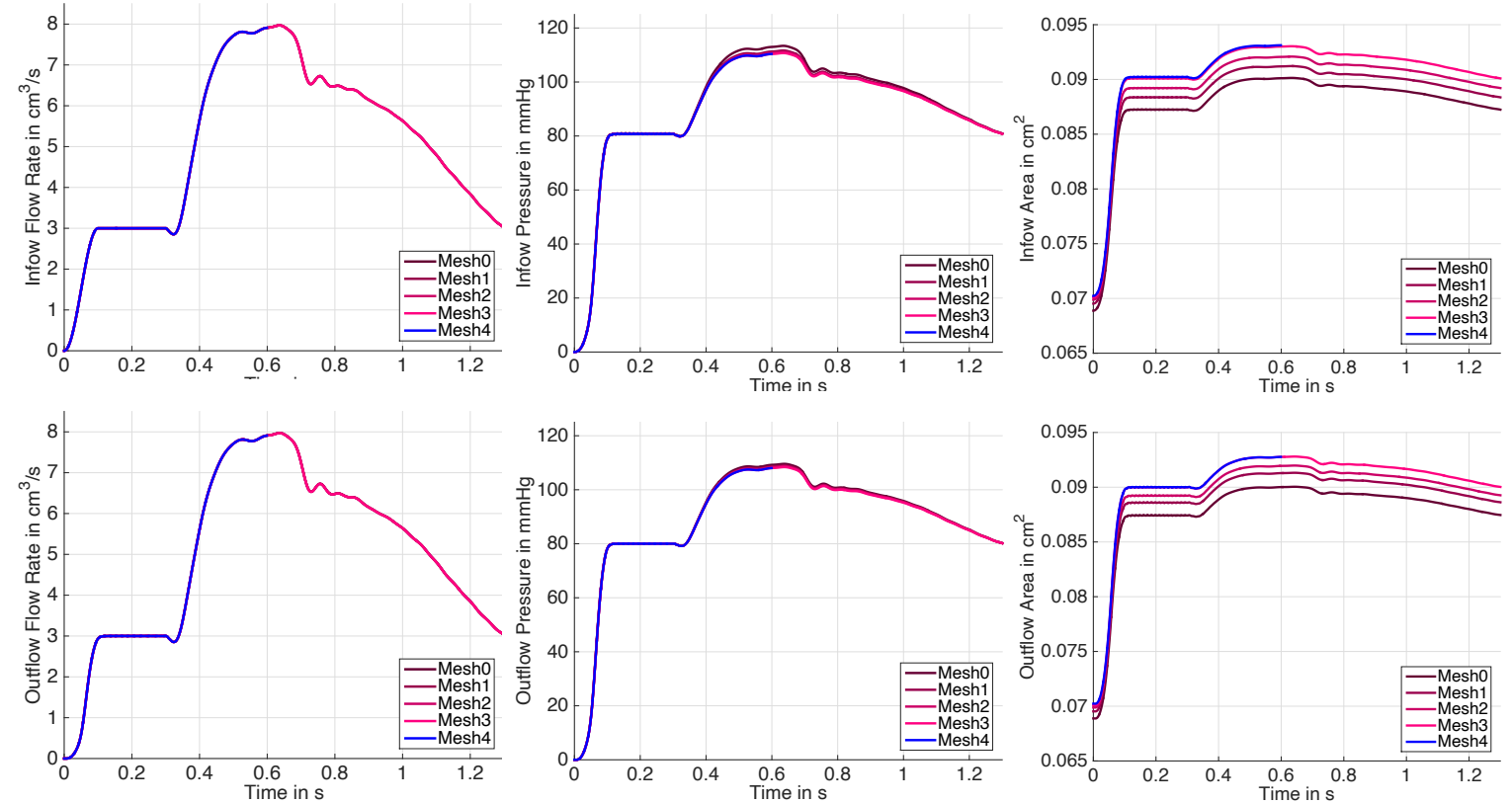

Fig. $44 \overline{\mathrm{F}}$ mesh convergence study for the hyperelastic material for the heartbeat. Flowrate (left), average pressure (middle), Lumen cross section area (right), over time at the inlet (top) and outlet (bottom); cf. Section 4.3. 

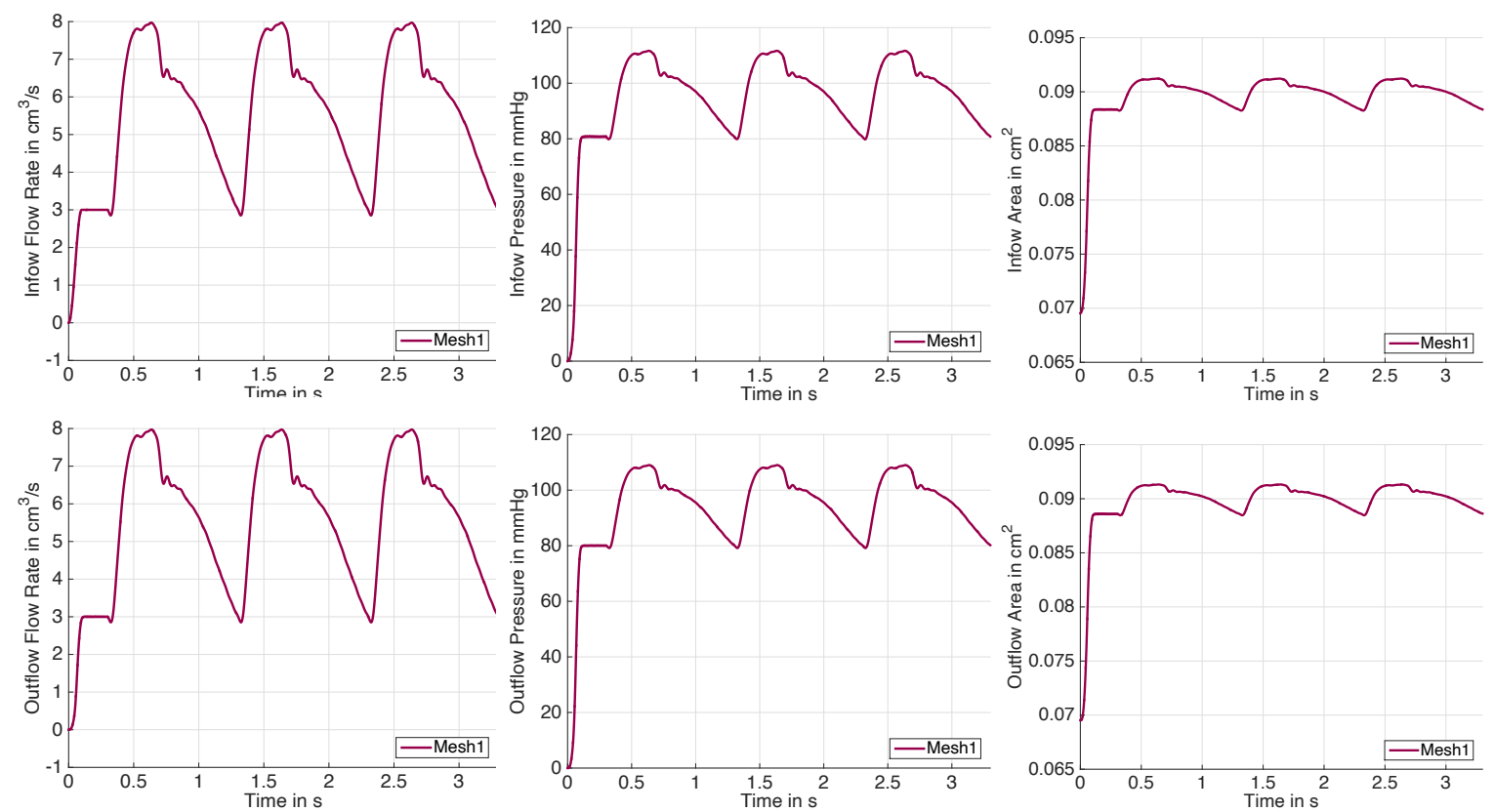

Fig. $45 \bar{F}$ mesh convergence study for the hyperelastic material for the heartbeat. Flowrate (left), average pressure (middle), Lumen cross section area (right), over time at the inlet (top) and outlet (bottom); cf. Section 4.3. 